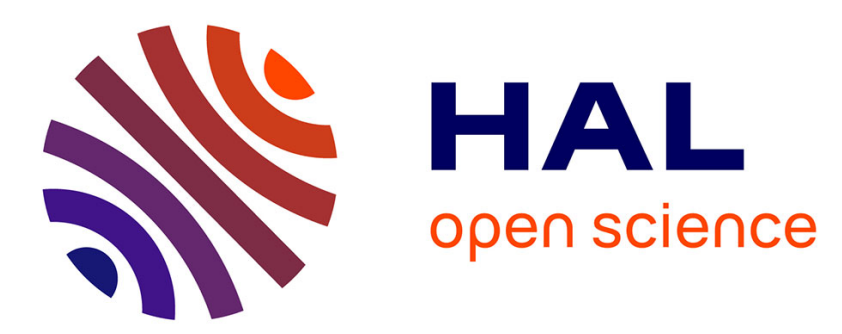

\title{
Limiting laws associated with Brownian motion perturbed by its maximum, minimum and local time II
}

\author{
Bernard Roynette, Pierre P. Vallois, Marc Yor
}

\section{To cite this version:}

Bernard Roynette, Pierre P. Vallois, Marc Yor. Limiting laws associated with Brownian motion perturbed by its maximum, minimum and local time II. Studia Scientiarum Mathematicarum Hungarica, 2006, 43 (3), pp.295-360. hal-00012705

\section{HAL Id: hal-00012705 https://hal.science/hal-00012705}

Submitted on 26 Oct 2005

HAL is a multi-disciplinary open access archive for the deposit and dissemination of scientific research documents, whether they are published or not. The documents may come from teaching and research institutions in France or abroad, or from public or private research centers.
L'archive ouverte pluridisciplinaire HAL, est destinée au dépôt et à la diffusion de documents scientifiques de niveau recherche, publiés ou non, émanant des établissements d'enseignement et de recherche français ou étrangers, des laboratoires publics ou privés. 


\title{
LIMITING LAWS ASSOCIATED WITH BROWNIAN MOTION PERTURBED BY ITS MAXIMUM, MINIMUM AND LOCAL TIME II
}

\author{
Bernard ROYNETTE ${ }^{(1)}$, Pierre VALLOIS $^{(1)}$ and Marc YOR ${ }^{(2),(3)}$
}

October 26, 2005

(1) Université Henri Poincaré, Institut de Mathématiques Elie Cartan, B.P. 239, F-54506 Vandœuvrelès-Nancy Cedex

(2) Laboratoire de Probabilités et Modèles Aléatoires, Universités Paris VI et VII - 4, Place Jussieu - Case 188 - F-75252 Paris Cedex 05.

(3) Institut Universitaire de France.

\begin{abstract}
Let $P_{0}$ denote the Wiener measure defined on the canonical space $\left(\Omega=\mathcal{C}\left(\mathbb{R}_{+}, \mathbb{R}\right)\right.$, $\left.\left(X_{t}\right)_{t \geq 0},\left(\mathcal{F}_{t}\right)_{t \geq 0}\right)$, and $\left(S_{t}\right)$ (resp. $\left(I_{t}\right)$ ), be the one sided-maximum (resp. minimum), $\left(L_{t}^{0}\right)$ the local time at 0 , and $\left(D_{t}\right)$ the number of down-crossings from $b$ to $a$ (with $b>a$ ). Let $\left.f: \mathbb{R} \times \mathbb{R}^{d} \longrightarrow\right] 0,+\infty[$ be a Borel function, and $\left(A_{t}\right)$ be a process chosen within the set: $\left\{\left(S_{t}\right) ;\left(S_{t}, t\right) ;\left(L_{t}^{0}\right) ;\left(S_{t}, I_{t}, L_{t}^{0}\right) ;\left(D_{t}\right)\right\}$, which consists of 5 elements. We prove a penalization result : under some suitable assumptions on $f$, there exists a positive $\left(\left(\mathcal{F}_{t}\right), P_{0}\right)$-martingale $\left(M_{t}^{f}\right)$, starting at 1 , such that :

$$
\lim _{t \rightarrow \infty} \frac{E_{0}\left[1_{\Gamma_{s}} f\left(X_{t}, A_{t}\right)\right]}{E_{0}\left[f\left(X_{t}, A_{t}\right)\right]}=Q_{0}^{f}\left(\Gamma_{s}\right):=E_{0}\left[1_{\Gamma_{s}} M_{s}^{f}\right], \quad \forall \Gamma_{s} \in \mathcal{F}_{s} \text { and } s \geq 0 .
$$

We determine the law of $\left(X_{t}\right)$ under the p.m. $Q_{0}^{f}$ defined on $\left(\Omega, \mathcal{F}_{\infty}\right)$ by $(0.1)$. For the $1^{\text {st }}, 3^{\text {rd }}$ and $5^{\text {th }}$ elements of the set, we prove first that $Q_{0}^{f}\left(A_{\infty}<\infty\right)=1$, and more generally $Q_{0}^{f}(0<g<\infty)=1$ where $g=\sup \left\{s>0, A_{s}=A_{\infty}\right\}$ (with the convention $\sup \emptyset=0$ ). Secondly, we split the trajectory of $\left(X_{t}\right)$ in two parts : $\left(X_{t}\right)_{0 \leq t \leq g}$ and $\left(X_{t+g}\right)_{t \geq 0}$, and we describe their laws under $Q_{0}^{f}$, conditionally on $A_{\infty}$. For the $2^{\text {nd }}$ and $4^{t h}$ elements, a similar result holds replacing $A_{\infty}$ by resp. $S_{\infty}, S_{\infty} \vee I_{\infty}$.
\end{abstract}

Key words and phrases : penalization, enlargement of filtration, maximum, minimum, local time, down-crossings.

AMS 2000 subject classifications : 60 B 10, 60 G 17, 60 G 40, 60 G 44, 60 J 25, 60 J 35, 60 J 55, $60 \mathrm{~J} 60,60 \mathrm{~J} 65$. 


\section{Introduction}

1.1 Let $\left(P_{x}\right)_{x \in \mathbb{R}}$ be the family of Wiener measures defined on the canonical space $\left(\Omega=\mathcal{C}\left(\mathbb{R}_{+}, \mathbb{R}\right)\right.$, $\left.\left(X_{t}\right)_{t \geq 0},\left(\mathcal{F}_{t}\right)_{t \geq 0}\right)$. To an $\left(\mathcal{F}_{t}\right)$-adapted, non negative process $\left(F_{t}\right)_{t \geq 0}$ such that $0<E_{x}\left(F_{t}\right)<\infty$, for any $t \geq 0, x \in \mathbb{R}$, we associate the probability measure $Q_{x, t}^{F}$ defined on $\left(\Omega, \mathcal{F}_{t}\right)$ as follows :

$$
Q_{x, t}^{F}\left(\Gamma_{t}\right)=\frac{1}{E_{x}\left[F_{t}\right]} E_{x}\left[1_{\Gamma_{t}} F_{t}\right], \quad \Gamma_{t} \in \mathcal{F}_{t} .
$$

A priori, the family $\left(Q_{x, t}^{F} ; t \geq 0\right)$ is not consistent : $Q_{x, t}^{F}\left(\Gamma_{s}\right)$ may be different from $Q_{x, s}^{F}\left(\Gamma_{s}\right)$ for $\Gamma_{s} \in \mathcal{F}_{s}$ and $s<t$; in fact, it is easy to see that $\left(Q_{x, t}^{F} ; t \geq 0\right)$ is consistent, if and only if $\widetilde{F}_{t}=F_{t} / E_{x}\left[F_{t}\right] ; t \geq 0$ is a $P_{x}$-martingale. When this condition holds, we write $Q_{x}^{\widetilde{F}}$ instead of $Q_{x, t}^{F}$. $Q_{x}^{\widetilde{F}}$ is well defined since $Q_{x}^{\widetilde{F}}\left(\Gamma_{s}\right)=E_{x}\left[1_{\Gamma_{s}} \widetilde{F}_{t}\right], \Gamma_{s} \in \mathcal{F}_{s}$ and $s \leq t$.

In a previous study (12], 13]), we have considered $F_{t}=\exp \left\{-\frac{1}{2} \int_{0}^{t} V\left(X_{s}\right) d s\right\}$, where $V: \mathbb{R} \longrightarrow \mathbb{R}_{+}$ is a Borel function. Our basic result was the following: under some suitable assumptions on $V$, for any given $s \geq 0$ and $\Gamma_{s}$ in $\mathcal{F}_{s}, Q_{x, t}^{F}\left(\Gamma_{s}\right)$ converges as $t \rightarrow \infty$, to $Q_{x}^{\widetilde{F}}\left(\Gamma_{s}\right)$ where $\widetilde{F}$ is the $\left(P_{x}\right)$-martingale

$$
\widetilde{F}_{t}=\frac{\varphi_{V}\left(X_{t}\right)}{\varphi_{V}\left(X_{0}\right)} F_{t}=\frac{\varphi_{V}\left(X_{t}\right)}{\varphi_{V}\left(X_{0}\right)} \exp \left\{-\frac{1}{2} \int_{0}^{t} V\left(X_{s}\right) d s\right\}
$$

and $\varphi_{V}$ is a "good" positive solution of the Sturm-Liouville equation $\varphi^{\prime \prime}=V \varphi$. The weak convergence of $Q_{x, t}^{F}$ to $Q_{x}^{\widetilde{F}}, t \rightarrow \infty$, is a direct consequence of the two following facts :

$$
\begin{aligned}
& \left(\left(X_{t}, F_{t}\right) ; t \geq 0\right) \text { is a Markov process, } \\
& E_{x}\left[F_{t}\right]=E_{x}\left[\exp \left\{-\frac{1}{2} \int_{0}^{t} V\left(X_{s}\right) d s\right\}\right]_{t \rightarrow \infty} t^{-k} \varphi_{V}(x), \text { for some } k \geq 0 .
\end{aligned}
$$

1.2 The goal of this paper is to deal with a more general setting by considering $F_{t}=f\left(X_{t}, A_{t}\right)$, where $\left.f: \mathbb{R} \times \mathbb{R}^{d} \longrightarrow\right] 0,+\infty\left[\right.$ is a Borel function, $\left(A_{t} ; t \geq 0\right)$ is $\left(\mathcal{F}_{t}\right)$ adapted and $\mathbb{R}^{d}$ - valued. We suppose moreover :

$$
\left(Y_{t} \stackrel{\text { (def) }}{=}\left(X_{t}, A_{t}\right) ; t \geq 0\right) \text { is a }\left(\left(P_{x}\right)_{x \in \mathbb{R}} ;\left(\mathcal{F}_{t}\right)_{t \geq 0}\right) \text {-Markov process. }
$$

Let $\left(\Lambda_{t}\right)_{t \geq 0}=\left(\Lambda_{t}\left(y, d y^{\prime}\right)=\Lambda_{t}\left(x, a ; d x^{\prime} d a^{\prime}\right) ; t \geq 0\right)$ be its semigroup (we denote $y=(x, a)$ and $\left.y^{\prime}=\left(x^{\prime}, a^{\prime}\right)\right)$.

To recover the setting of [12], [13] recalled above, it suffices to choose $d=1, f(x, a)=e^{-a / 2}$ and $A_{t}=\int_{0}^{t} V\left(X_{s}\right) d s$. Let $\left(Q_{x, t}^{F} ; x \in \mathbb{R}, t \geq 0\right)$ be the family of p.m. (E probability measures) associated with $\left(F_{t}\right)_{t \geq 0}$ :

$$
Q_{x, t}^{F}\left(\Gamma_{t}\right)=\frac{1}{E_{x}\left[F_{t}\right]} E_{x}\left[1_{\Gamma_{t}} F_{t}\right]=\frac{1}{E_{x}\left[f\left(X_{t}, A_{t}\right)\right]} E_{x}\left[1_{\Gamma_{t}} f\left(X_{t}, A_{t}\right)\right], \Gamma_{t} \in \mathcal{F}_{t}, t \geq 0 .
$$

We now present a "meta-theorem", i.e. a statement which will hold in great generality, so much so that our remaining study shall consist in verifying that the hypotheses of Theorem 1.1 hold in various cases. 
Theorem 1.1 Let $y_{0}=\left(x_{0}, a_{0}\right)$. We suppose $P_{x_{0}}\left(A_{0}=a_{0}\right)=1$ and

$$
M_{s}\left(y_{0}, f ; y\right):=\lim _{t \rightarrow \infty} \frac{\Lambda_{t-s}(f)(y)}{\Lambda_{t}(f)\left(y_{0}\right)}
$$

exists, for any $s \geq 0$ and $y=(x, a) \in \mathbb{R} \times \mathbb{R}^{d}$,

$$
\frac{\Lambda_{t-s}(f)(y)}{\Lambda_{t}(f)\left(y_{0}\right)} \leq C(s, y) ; \forall t>s
$$

where $E_{x_{0}}\left[C\left(s, Y_{s}\right)\right]<\infty$.

Then :

1. $\left(M_{s}:=M_{s}\left(y_{0}, f ; Y_{s}\right) ; s \geq 0\right)$ is a non-negative $P_{x_{0}}$-martingale, and $M_{0}=1, P_{x_{0}}$-a.s.

2. $Q_{x_{0}, t}^{F}$ converges weakly to $Q_{x_{0}}^{M}, t \rightarrow \infty$ (i.e. $\lim _{t \rightarrow \infty} Q_{x_{0}, t}^{F}\left(\Gamma_{s}\right)=Q_{x_{0}}^{M}\left(\Gamma_{s}\right), \forall \Gamma_{s} \in \mathcal{F}_{s}, \forall s>0$ ), where :

$$
Q_{x_{0}}^{M}\left(\Gamma_{s}\right)=E_{x_{0}}\left[1_{\Gamma_{s}} M_{s}\right], \Gamma_{s} \in \mathcal{F}_{s} ; s \geq 0 .
$$

By definition, the p.m. $Q_{x_{0}, t}^{F}$ is absolutely continuous on $\left(\Omega, \mathcal{F}_{t}\right)$, with respect to the Wiener measure. The Radon-Nikodym density $F_{t} / E\left[F_{t}\right]$ may be interpreted as a weight or a penalization as it is done in statistical mechanics. In the sequel, Theorem 1.1 will be refered to as a penalization principle.

Proof of Theorem 1.1 Let $s>0$ and $\Gamma_{s} \in \mathcal{F}_{s}$ fixed. Using the definition of $Q_{x, t}^{F}\left(\Gamma_{s}\right)$ and the Markov property we have :

$$
Q_{x_{0}, t}^{F}\left(\Gamma_{s}\right)=E_{x_{0}}\left[1_{\Gamma_{s}} \frac{\Lambda_{t-s}(f)\left(X_{s}, A_{s}\right)}{\Lambda_{t}(f)\left(x_{0}, A_{0}\right)}\right]
$$

Property (1.7) and inequality (1.8) allow us to apply the dominated convergence theorem :

$$
\lim _{t \rightarrow \infty} Q_{x_{0}, t}^{F}\left(\Gamma_{s}\right)=Q_{x_{0}}^{M}\left(\Gamma_{s}\right),
$$

where $M_{s}=M_{s}\left(y_{0}, f ; Y_{s}\right)$.

It is clear that $M_{0}\left(y_{0}, f ; Y_{0}\right)=1$; consequently $P_{x_{0}}\left(M_{0}=1\right)=1$.

Let $0 \leq s<s^{\prime}$ and $\Gamma_{s} \in \mathcal{F}_{s}$; since $\Gamma_{s} \in \mathcal{F}_{s^{\prime}}$ we have : $E_{x_{0}}\left[1_{\Gamma_{s}} M_{s}\right]=E_{x_{0}}\left[1_{\Gamma_{s}} M_{s^{\prime}}\right]$. This means that $\left(M_{t}\right)$ is a $P_{x_{0}}$-martingale.

1.3 In this paper, we investigate four cases of examples involving respectively for $\left(A_{t}\right)$ :

- the unilateral maximum (resp. minimum) $S_{t}$ (resp. $\left.I_{t}\right): S_{t}=\max _{0 \leq u \leq t} X_{u}$ (resp. $I_{t}=-\min _{0 \leq u \leq t} X_{u}$ ). We also consider, in the same case study the two-dimensional process $\left(S_{t}, t\right)$.

- $\left(L_{t}^{0} ; t \geq 0\right)$ the local time at 0 of $\left(X_{t}\right)_{t \geq 0}$.

- The triplet $\left(\left(S_{t}, I_{t}, L_{t}^{0}\right) ; t \geq 0\right)$.

- $\left(D_{t} ; t \geq 0\right)$ the number of down-crossings of $X$ from level $b$ to level $a$.

We observe that, in all cases, the function $M_{s}\left(y_{0}, f ; y\right)$ may be written as :

$$
\frac{M(f ; y)}{M\left(f ; y_{0}\right)} e^{\alpha s}
$$

for some function $M$ and some $\alpha \in \mathbb{R}$; in fact $\alpha=0$, except for the case 1, b), as shown below.

Since $s \rightarrow M_{s}\left(y_{0}, f ; Y_{s}\right)$ is a $P_{x_{0}}$-martingale, it is clear that $s \rightarrow M\left(f ; Y_{s}\right) e^{\alpha s}$ is also a $P_{x_{0}}$-martingale. The results are summarized in the following Table: 


\begin{tabular}{|c|c|c|c|c|}
\hline Cases & $A_{t}$ & $F_{t}$ & $M\left(f ; Y_{t}\right) e^{\alpha t}$ & Theorem \\
\hline \multirow{2}{*}{1} & $a) \quad S_{t}$ & $\varphi\left(S_{t}\right)$ & $M_{t}^{\varphi}$ & 3.6 \\
\cline { 2 - 5 } & $b)\left(S_{t}, t\right)$ & $\varphi\left(S_{t}\right) e^{\lambda\left(S_{t}-X_{t}\right)}$ & $M_{t}^{\lambda, \varphi}$ & 3.9 \\
\hline \multirow{2}{*}{2} & $L_{t}^{0}$ & $h^{+}\left(L_{t}^{0}\right) 1_{\left\{X_{t}>0\right\}}+h^{-}\left(L_{t}^{0}\right) 1_{\left\{X_{t}<0\right\}}$ & $M_{t}^{h^{+}, h^{-}}$ & 3.13 \\
\hline 3 & $\left(S_{t}, I_{t}, L_{t}^{0}\right)$ & $A_{\nu}\left(S_{t}, I_{t}, L_{t}^{0}\right)$ & $M_{t}^{\nu}$ & 3.18 \\
\hline & $D_{t}$ & $\Delta G\left(D_{t}\right)$ & $M_{t}^{\downarrow, G}$ & 3.21 \\
\hline
\end{tabular}

These four cases will be treated in sections 3.13 3.4 respectively.

Let us describe the martingale $M\left(f ; Y_{t}\right) e^{\alpha t}$ for each case.

Case 1. a) We have :

$$
M_{t}^{\varphi}=\left(S_{t}-X_{t}\right) \varphi\left(S_{t}\right)+1-\Phi\left(S_{t}\right)
$$

where $\varphi: \mathbb{R} \mapsto \mathbb{R}_{+}$is bounded, $\int_{\mathbb{R}} \varphi(u) d u=1$ and $\Phi(x)=\int_{-\infty}^{x} \varphi(u) d u$.

b) More generally, for $\lambda>0$ :

$$
M_{t}^{\lambda, \varphi}=\left\{\left(1-\Phi\left(S_{t}\right)\right) \cosh \left(\lambda\left(S_{t}-X_{t}\right)\right)+\varphi\left(S_{t}\right) \frac{\sinh \left(\lambda\left(S_{t}-X_{t}\right)\right)}{\lambda}\right\} e^{-\lambda^{2} t / 2},
$$

where $\psi: \mathbb{R} \mapsto \mathbb{R}_{+}, \xi_{\lambda}(x)=e^{\lambda x} 1_{\{x<0\}}, 1-\Phi=\psi * \xi_{\lambda}$ and $\varphi=\Phi^{\prime}$.

Both families $\left(M_{t}^{\varphi}, M_{t}^{\lambda, \varphi}\right)$ were intensively used in 1] to solve Skorokhod's problem for Brownian motion. The class $\left(M_{t}^{\varphi}\right)$ (resp. $\left(M_{t}^{\lambda, \varphi}\right)$ is defined in detail in Proposition 3.1 (resp. Proposition 3.3) and the special case of the related meta-theorem in Theorem 3.6 (resp. Theorem 3.9).

Case 2. Let $h^{+}, h^{-}: \mathbb{R}_{+} \longrightarrow \mathbb{R}_{+}$be two Borel, bounded functions, $H(l):=\frac{1}{2} \int_{0}^{l}\left(h^{+}(u)+h^{-}(u)\right) d u$. We suppose $H(+\infty)=1 .\left(M_{t}^{h^{+}, h^{-}}\right)$is the martingale :

$$
M_{t}^{h^{+}, h^{-}}=X_{t}^{+} h^{+}\left(L_{t}^{0}\right)+X_{t}^{-} h^{-}\left(L_{t}^{0}\right)+1-H\left(L_{t}^{0}\right) .
$$

These martingales appear in $[7]$.

The limit theorem associated with $A_{t}=L_{t}^{0}$ is stated, only with $x_{0}=0$, in Theorem 3.13 .

Case 3. Let $\nu$ be a p.m. on $[\alpha, \infty[\times[\alpha, \infty[$ for some $\alpha>0$ and :

$$
A_{\nu}(s, i, l)=\int_{\mathbb{R}_{+}^{2}} e^{\frac{1}{2}\left(\frac{1}{a}+\frac{1}{b}\right) l} 1_{\{a \geq s, b \geq i\}} \nu(d a, d b) ; s, i, l \geq 0 .
$$

$\left(M_{t}^{\nu}\right)$ is the martingale :

$$
M_{t}^{\nu}=\int_{\mathbb{R}_{+}^{2}}\left(1-\frac{X_{t}^{+}}{a}\right)\left(1-\frac{X_{t}^{-}}{b}\right) \exp \left\{\frac{1}{2}\left(\frac{1}{a}+\frac{1}{b}\right) L_{t}^{0}\right\} 1_{\left\{S_{t} \leq a, I_{t} \leq b\right\}} \nu(d a, d b) .
$$

Some properties of the family $\left(M_{t}^{\nu}\right)$ are given in Proposition 3.16, and the penalization principle is stated in Theorem 3.18 .

Case 4. Let $D_{t}$ be the number of down-crossings from $b$ to $a$, achieved by $\left(X_{t}\right)$ up to time $t$, and $(G(n))_{n \geq 0}$ be a decreasing sequence of positive numbers such that $G(0)=1$ and $\lim _{n \rightarrow \infty} G(n)=0$. Then

$$
M_{t}^{\downarrow, G}=\sum_{n \geq 0}\left\{1_{\left[\sigma_{2 n}, \sigma_{2 n+1}[\right.}(t)\left(\frac{G(n)}{2}\left(1+\frac{b-X_{t}}{b-a}\right)+\frac{G(n+1)}{2} \frac{X_{t}-a}{b-a}\right)\right.
$$




$$
\left.+1_{\left[\sigma_{2 n+1}, \sigma_{2 n+2}[\right.}(t)\left(\frac{G(n+1)}{2}\left(1+\frac{b-X_{t}}{b-a}\right)+\frac{G(n)}{2} \frac{X_{t}-a}{b-a}\right)\right\} .
$$

where $\sigma_{0}=0$ and $\left(\sigma_{n}\right)_{n \geq 1}$ is defined inductively as follows : $\sigma_{1}=\inf \left\{t \geq 0 ; X_{t}>b\right\}, \sigma_{2}=\inf \{t \geq$ $\left.0, X_{t}<a\right\}, \sigma_{2 n+1}=\sigma_{1} \circ \theta_{\sigma_{2 n}}, \sigma_{2 n+2}=\sigma_{2} \circ \theta_{\sigma_{2 n+1}}$, where $\left(\theta_{u}\right)_{u \geq 0}$ denotes the family of shift operators on the canonical space.

The corresponding case of the meta-theorem is stated in Theorem 3.21 .

1.4 Theorem 1.1 leads naturally to ask for a description of the law of $\left(X_{t}\right)_{t \geq 0}$ under $Q_{x_{0}}^{M}$. Since $\left(M_{t}\right)_{t \geq 0}$ is a strictly positive $\left(P_{x_{0}}\right)$-martingale, it may be written as an exponential martingale :

$$
M_{t}=\mathcal{E}(J)_{t}=\exp \left\{\int_{0}^{t} J_{s} d X_{s}-\frac{1}{2} \int_{0}^{t} J_{s}^{2} d s\right\}
$$

for some adapted process $\left(J_{t}\right)_{t \geq 0}$.

Girsanov's Theorem implies that $\left(\beta_{t} ; t \geq 0\right)=\left(X_{t}-\int_{0}^{t} J_{s} d s ; t \geq 0\right)$ is a $Q_{x_{0}}^{M}$-Brownian motion.

Suppose that $(x, a) \longrightarrow M(f ; x, a)$ is of class $C^{2,1}$ and $\left(A_{t}\right)$ has bounded variation. Then by Ito's formula we obtain :

$$
J_{t}=\alpha+\frac{\partial}{\partial x}\left(\log \left(M\left(f ; X_{t}, A_{t}\right)\right)\right) .
$$

Consequently $\left(X_{t}\right)$ solves the following stochastic differential equation :

$$
X_{t}=\beta_{t}+\int_{0}^{t}\left(\alpha+\frac{\frac{\partial M}{\partial x}}{M}\left(f ; X_{s}, A_{s}\right)\right) d s .
$$

We may recover (1.15) in a different manner. Suppose for simplicity that $\alpha=0$. It is clear that $\left(M\left(f ; Y_{s}\right) ; s \geq 0\right)$ is a non-negative $P_{x_{0}}$-martingale. In other words, $y \rightarrow M(f ; y)$ is a non-negative harmonic function with respect to $\left(Y_{t}\right)_{t \geq 0}$, under $P_{x_{0}}$. In particular $\left(Y_{t}\right)_{t \geq 0}$ is a $Q_{x_{0}}^{M}$-Markov process with semigroup :

$$
\Lambda_{t}^{M}(g)(y)=\frac{\Lambda_{t}(g M(f ; \cdot))(y)}{M(f ; y)} .
$$

If $(x, a) \mapsto M(f ; x, a)$ is of class $C^{2,1}$, then the generator associated with the semigroup $\left(\Lambda_{t}^{M}\right)$ is

$$
\frac{1}{2} \frac{\partial^{2}}{\partial x^{2}}+\frac{\frac{\partial M}{\partial x}}{M}(f ; x, a) \frac{\partial}{\partial x}+\frac{\frac{\partial M}{\partial a}}{M}(f ; x, a) \frac{\partial}{\partial a} .
$$

This gives a new proof of $(1.15)$.

However in our four classes of examples we observe that the drift term in (1.15) is explicit but complicated and therefore does not allow to identify directly the law of $\left(X_{t}\right)$ under $Q_{x_{0}}^{M}$. Suppose that $\left(A_{t}\right)_{t \geq 0}$ is a one-dimensional process, $Q_{x_{0}}\left(A_{0}=0\right)=1$ and $t \rightarrow A_{t}$ is continuous and non-decreasing. The r.v. $A_{\infty}$, which may be infinite, plays a central role in our approach. We claim that we may compute the distribution function of $A_{\infty}$ under $Q_{x_{0}}$. Let $\left(A_{t}^{-1}\right)_{t \geq 0}$ be the right inverse of $s \mapsto A_{s}$, i.e. $: A_{t}^{-1}=\inf \left\{s \geq 0, A_{s}>t\right\}$. We have :

$$
Q_{x_{0}}\left(A_{t}>\alpha\right)=Q_{x_{0}}\left(A_{\alpha}^{-1}<t\right)=E_{x_{0}}\left[1_{\left\{A_{\alpha}^{-1}<t\right\}} M_{t}\right]=E_{x_{0}}\left[1_{\left\{A_{\alpha}^{-1}<t\right\}} M_{A_{\alpha}^{-1}}\right] .
$$

Note that the last equality follows from the optional stopping theorem.

Since $M_{A_{\alpha}^{-1}} \geq 0$, taking $t \rightarrow \infty$, in the previous expression, we get :

$$
Q_{x_{0}}\left(A_{\infty}>\alpha\right)=E_{x_{0}}\left[1_{\left\{A_{\alpha}^{-1}<\infty\right\}} M_{A_{\alpha}^{-1}}\right]=E_{x_{0}}\left[1_{\left\{A_{\alpha}^{-1}<\infty\right\}} M\left(x_{0}, 0, f ; X_{A_{\alpha}^{-1}}, \alpha\right)\right] .
$$

In cases 1 (with $A=S$ only), 2 and 4 , we prove that $Q_{x_{0}}\left(A_{\infty}<\infty\right)=1$, and more generally $Q_{x_{0}}(0<g<\infty)=1$ where $g=\sup \left\{s>0, A_{s}=A_{\infty}\right\}$ (with the convention $\sup \emptyset=0$ ). To describe the law of $\left(X_{t}\right)$ under $Q_{x_{0}}$, it is convenient to split the whole trajectory in two parts : $\left(X_{t}\right)_{0 \leq t \leq g}$ and 
$\left(X_{t+g}\right)_{t \geq 0}$. We observe that the random time $g$ is not a $\left(\mathcal{F}_{t}\right)$-stopping time but it is a last exit time. Using the technique of enlargement of filtrations, we are able to describe the law of $\left(X_{t}\right)_{0 \leq t \leq g}$ and $\left(X_{t+g}\right)_{t \geq 0}$ under $Q_{x_{0}}^{M}$, conditionally to $A_{\infty}$ (see Theorems 4.6, 4.8 and 4.11).

As for case 3 (i.e. $\left.A_{t}=\left(S_{t}, I_{t}, L_{t}^{0}\right)\right)$, we prove that $Q_{0}\left(X_{\infty}^{*}<\infty\right)=1$, with $X_{\infty}^{*}=\sup _{t \geq 0}\left|X_{t}\right|=S_{\infty} \vee I_{\infty}$.

However $Q_{0}\left(L_{\infty}^{0}=\infty\right)=1$. Conditionally on $X_{\infty}^{*}$, the law of $\left(X_{t}\right)_{t \geq 0}$ is given in Theorem 4.9, via a path decomposition at time $g=\sup \left\{t \geq 0 ;\left|X_{t}\right|=X_{\infty}^{*}\right\}$.

1.5 In Section 5, we recover previous results, using a direct approach based on a disintegration of $Q_{0}$. We exhibit a family $\left(Q_{0}^{(a)} ; a \in \mathbb{R}^{d}\right)$ of p.m. on $\left(\Omega, \mathcal{F}_{\infty}\right)$ such that :

$$
Q_{0}(\cdot)=\int_{\mathbb{R}^{d}} Q_{0}^{(a)}(\cdot) \mu(d a),
$$

where in cases 1 (resp. 2,3), $\mu$ is the law of $S_{\infty}$ (resp. $\left.L_{\infty}^{0},\left(S_{\infty}, I_{\infty}, L_{\infty}^{0}\right)\right)$.

We are able to determine the law of $\left(X_{t}\right)$ under $Q_{0}^{(a)}$, for any $a$. Moreover this distribution does not depend on $f$.

1.6 Finally, in Section 6, we present several other directions of research, which we have now begun to investigate and will be the subject of a future publication.

\section{Notation}

In the sequel of the paper we shall use intensively the following notation and conventions.

- $\left(\Omega=\mathcal{C}\left(\mathbb{R}_{+}, \mathbb{R}\right),\left(X_{t}\right)_{t \geq 0},\left(\mathcal{F}_{t}\right)_{t \geq 0}\right)$ is the canonical space, with $\left(X_{t}\right)_{t \geq 0}$ the coordinate maps : $X_{t}(\omega)=\omega(t)$ and $\mathcal{F}_{t}=\sigma\left\{X_{s} ; 0 \leq s \leq t\right\} ; t \geq 0$.

- $\left(P_{x}\right)_{x \in \mathbb{R}}$ is the family of Wiener measures on the canonical space $\Omega$ : under $P_{x},\left(X_{t}\right)_{t \geq 0}$ is a one-dimensional Brownian motion started at $x$.

Let $\lambda$ and $x$ be two real numbers. We denote by $P_{x}^{(\lambda)}$ the p.m. on the canonical space, under which $\left(X_{t}\right)_{t \geq 0}$ is a Brownian motion with drift $\lambda$, started at $x$. Obviously $P_{x}=P_{x}^{(0)}$ and $\left(X_{t}\right)_{t \geq 0}$ is distributed under $P_{x}^{(\lambda)}$ as $\left(X_{t}+\lambda t\right)_{t \geq 0}$ under $P_{x}$.

If $Q$ is a probability measure (p.m.) on $\Omega$, the expectation with respect to $Q$ is denoted $E_{Q}$. However if $Q=P_{x}$, we shall write $E_{x}$ for $E_{P_{x}}$ for simplicity.

- $\left(\theta_{t}\right)_{t \geq 0}$ is the family of shift operators from $\Omega$ to $\Omega$, defined by $\theta_{t}(\omega)(s)=\omega(t+s) ; s \geq 0, t \geq 0$.

- For any $a \in \mathbb{R}, T_{a}$ is the first hitting time of level $a$, namely : $T_{a}=\inf \left\{t \geq 0, X_{t}=a\right\}$. We adopt the convention $\inf \{\emptyset\}=+\infty$.

- $\left(S_{t} ; t \geq 0\right)$ (resp. $\left.\left(-I_{t} ; t \geq 0\right)\right)$, is the one-sided maximum (resp. minimum) :

$$
S_{t}=\sup _{0 \leq u \leq t} X_{u} ; I_{t}=-\inf _{0 \leq u \leq t} X_{u} .
$$

- The bilateral maximum $\left(X_{t}^{*}\right)_{t \geq 0}$ is the process :

$$
X_{t}^{*}=S_{t} \vee I_{t}=\sup _{0 \leq u \leq t}\left|X_{u}\right| .
$$

- $\left(L_{t}^{x} ; x \in \mathbb{R}, t \geq 0\right)$ is the jointly continuous family of local times associated with $\left(X_{t}\right)_{t \geq 0}$. For simplicity we write $\left(L_{t}\right)_{t \geq 0}$ instead of $\left(L_{t}^{0}\right)_{t \geq 0}$.

- If $J$ is a predictable process (with respect to $\left(\mathcal{F}_{t}\right)$ ) such that $\int_{0}^{t} J_{s}^{2} d s<\infty P_{x}$ a.s. for every $t$, then we denote by $\mathcal{E}(J)_{t}$ the $P_{x}$-local martingale :

$$
\exp \left\{\int_{0}^{t} J_{s} d X_{s}-\frac{1}{2} \int_{0}^{t} J_{s}^{2} d s\right\}, t \geq 0 .
$$




\section{Penalization and associated martingales}

\subsection{Case 1 : The one-sided maximum}

In this section, we consider two families of local martingales $\left(M_{t}^{\varphi}\right)$ and $\left(M_{t}^{\lambda, \varphi}\right)$ involving the onesided maximum. These local martingales are well-known : they play a prominent role in Azéma-Yor's solution of Skorokhod's problem studied in [1]. We will show (see Theorems 3.6 and 3.9) that a sub-class of the previous local martingales appears naturally after a penalization procedure.

\subsubsection{The local martingales associated with the one-sided maximum}

Let us start with local martingales of the form $H\left(X_{t}, S_{t}\right)$, and more generally $H\left(X_{t}, S_{t}, t\right)$, for some function $H$.

Proposition 3.1 1. Let $\varphi: \mathbb{R} \longrightarrow] 0,+\infty[$ be a Borel function such that

$$
\begin{gathered}
\int_{-\infty}^{\cdot} \varphi(u) d u<\infty \text {, and define } \Phi(s)=\int_{-\infty}^{s} \varphi(u) d u . \text { Then : } \\
M_{t}^{\varphi}:=\left(S_{t}-X_{t}\right) \varphi\left(S_{t}\right)+1-\Phi\left(S_{t}\right),
\end{gathered}
$$

is a $P_{x}$-martingale, $M_{0}^{\varphi}=1-\Phi(x), P_{x}$ a.s., and:

$$
M_{t}^{\varphi}=1-\Phi(x)-\int_{0}^{t} \varphi\left(S_{u}\right) d X_{u}
$$

2. Suppose moreover :

$$
\int_{\mathbb{R}} \varphi(u) d u=1
$$

Then under $P_{x}, M_{t}^{\varphi}>0$ and $M_{t}^{\varphi}=(1-\Phi(x)) \mathcal{E}\left(J^{\varphi}\right)_{t}$ where :

$$
J_{t}^{\varphi}=-\frac{\varphi\left(S_{t}\right)}{M_{t}^{\varphi}}=-\frac{\varphi\left(S_{t}\right)}{\left(S_{t}-X_{t}\right) \varphi\left(S_{t}\right)+1-\Phi\left(S_{t}\right)} .
$$

Remark 3.2 1. Note that the local martingale $\left(M_{t}^{\varphi}\right)$ is actually a martingale since :

$$
\sup _{0 \leq u \leq t}\left|M_{u}^{\varphi}\right| \leq 1+2\|\varphi\|_{\infty} \sup _{0 \leq u \leq t}\left|X_{u}\right| .
$$

2. Taking $\varphi(u)=\frac{1}{a} 1_{[0, a]}(u)$, with $a>0$, we obtain : $\Phi(x)=\frac{1}{a}(x \wedge a)$ and $M_{t}^{\varphi}=1-\frac{X_{t \wedge T_{a}}}{a}$.

We now recall the definition of Kennedy's martingales, which also played some role in the computation of the laws of the stopping times studied in [1] .

Proposition 3.3 Let $\lambda>0, \varphi: \mathbb{R} \longrightarrow \mathbb{R}$ be a locally integrable function, $\Phi$ be any primitive of $\varphi$ $\left(\varphi(x)=\Phi^{\prime}(x)\right)$. Let $\left(M_{t}^{\lambda, \varphi}\right)$ be the process :

$$
M_{t}^{\lambda, \varphi}:=\left\{\left(1-\Phi\left(S_{t}\right)\right) \cosh \left(\lambda\left(S_{t}-X_{t}\right)\right)+\varphi\left(S_{t}\right) \frac{\sinh \left(\lambda\left(S_{t}-X_{t}\right)\right)}{\lambda}\right\} e^{-\lambda^{2} t / 2},
$$

Then :

1. $\left(M_{t}^{\lambda, \varphi}\right)$ is a $P_{x}$-local martingale. Under $P_{x}, M_{0}^{\lambda, \varphi}=1-\Phi(x)$ and :

$$
M_{t}^{\lambda, \varphi}=1-\Phi(x)-\int_{0}^{t}\left\{-\lambda\left(1-\Phi\left(S_{u}\right)\right) \sinh \left(\lambda\left(S_{u}-X_{u}\right)\right)+\varphi\left(S_{u}\right) \cosh \left(\lambda\left(S_{u}-X_{u}\right)\right)\right\} e^{-\lambda^{2} u / 2} d X_{u} .
$$


2. Let $x_{0} \in \mathbb{R}$. Then $M_{t}^{\lambda, \varphi} \geq 0, \forall t \geq 0, P_{x_{0}}$ a.s. if and only if there exists a Borel function $\psi: \mathbb{R} \mapsto[0, \infty[$ and a non-negative constant $\kappa$ such that the two following conditions hold:

$$
\begin{aligned}
& \int_{x_{0}}^{\infty} \psi(z) e^{-\lambda z} d z<\infty \\
& 1-\Phi(y)=e^{\lambda y}\left(\kappa+\int_{y}^{\infty} \psi(z) e^{-\lambda z} d z\right), y \geq x_{0} .
\end{aligned}
$$

Assuming that (3.7) and (3.8) hold, then $\left(M_{t}^{\lambda, \varphi}\right)$ is a $P_{x_{0}}$-martingale.

3. Let $\psi: \mathbb{R} \mapsto[0, \infty[$ be a Borel function satisfying :

$$
\int_{x}^{\infty} \psi(z) e^{-\lambda z} d z<\infty, \quad \forall x \in \mathbb{R}
$$

Let $\Phi: \mathbb{R} \mapsto \mathbb{R}$ be the function :

$$
\Phi(y)=1-e^{\lambda y} \int_{y}^{\infty} \psi(z) e^{-\lambda z} d z, y \in \mathbb{R} .
$$

Then :

$$
\varphi(y)=\Phi^{\prime}(y)=\psi(y)-\lambda e^{\lambda y} \int_{y}^{\infty} \psi(z) e^{-\lambda z} d z,
$$

$\left(M_{t}^{\lambda, \varphi}\right)$ is a $P_{x}$-martingale and:

$$
M_{t}^{\lambda, \varphi}=\left\{\psi\left(S_{t}\right) \frac{\sinh \left(\lambda\left(S_{t}-X_{t}\right)\right)}{\lambda}+e^{\lambda X_{t}} \int_{S_{t}}^{\infty} \psi(z) e^{-\lambda z} d z\right\} e^{-\lambda^{2} t / 2}
$$

Suppose moreover $\psi>0$, then under $P_{x}: M_{t}^{\lambda, \varphi}>0$ and $M_{t}^{\lambda, \varphi}=(1-\Phi(x)) \mathcal{E}\left(J^{\lambda, \varphi}\right)_{t}$, where :

$$
J_{t}^{\lambda, \varphi}=-\lambda \frac{\varphi\left(S_{t}\right) \cosh \left(\lambda\left(S_{t}-X_{t}\right)\right)+\lambda\left(1-\Phi\left(S_{t}\right)\right) \sinh \left(\lambda\left(S_{t}-X_{t}\right)\right)}{\lambda\left(1-\Phi\left(S_{t}\right)\right) \cosh \left(\lambda\left(S_{t}-X_{t}\right)\right)+\varphi\left(S_{t}\right) \sinh \left(\lambda\left(S_{t}-X_{t}\right)\right)} .
$$

Proof of Proposition 3.3 1) If $\varphi$ is of class $C^{1}$, then point 1) of Proposition 3.3 is a direct consequence of Itô's formula and the fact that $d S$ is carried by $\{S-X=0\}$. The general case follows from the monotone class theorem.

2) Let us investigate the positivity of $M_{t}^{\lambda, \varphi}$, under $P_{x_{0}}$. It is clear that : $M_{t}^{\lambda, \varphi} \geq 0, \forall t \geq 0, P_{x_{0}}$ a.s. is equivalent to :

$$
(1-\Phi(y)) \cosh (\lambda(y-x))+\varphi(y) \frac{\sinh (\lambda(y-x))}{\lambda} \geq 0,
$$

for all $x$ and $y$ such that $y \geq x_{0}$ and $x \leq y$.

Setting $u=y-x$ and rewriting sinh and cosh in terms of exponential functions, it is easy to check that the previous inequality is equivalent to:

$$
e^{2 \lambda u}\left((1-\Phi(y))+\frac{\varphi(y)}{\lambda}\right)+1-\Phi(y)-\frac{\varphi(y)}{\lambda} \geq 0, \quad \forall u \geq 0, y \geq x_{0} .
$$

Since $\alpha Y_{1}+Y_{2} \geq 0, \forall \alpha \geq 1$ iff $Y_{1} \geq 0$ and $Y_{1}+Y_{2} \geq 0$, the previous inequality is equivalent to :

$$
1-\Phi(y)+\frac{\varphi(y)}{\lambda} \geq 0, \quad \forall y \geq x_{0},
$$


and

$$
\Phi(y) \leq 1, \quad \forall y \geq x_{0}
$$

Consequently the function $\psi$ defined by

$$
\psi(y)=\lambda(1-\Phi(y))+\varphi(y), y \geq x_{0},
$$

takes its values in $[0, \infty[$.

Recall that $\Phi^{\prime}=\varphi$, hence (3.16) may be interpreted as an ordinary linear differential equation in $\Phi$, which is easily solved :

$$
\Phi(y)=1+e^{\lambda y}\left(\kappa_{0}+\int_{x_{0}}^{y} \psi(z) e^{-\lambda z} d z\right), y \geq x_{0},
$$

where $\kappa_{0}$ is a constant.

Since (3.14) and (3.17) are equivalent, it remains to deal with (3.15). Obviously this inequality is equivalent to :

$$
\kappa_{0}+\int_{x_{0}}^{y} \psi(z) e^{-\lambda z} d z \leq 0, \forall y \geq x_{0} .
$$

The function $\psi$ being non-negative, this last condition is equivalent to (3.7) and

$$
\kappa=-\kappa_{0}-\int_{x_{0}}^{\infty} \psi(z) e^{-\lambda z} d z \geq 0
$$

Relation (3.8) is a direct consequence of (3.17).

3) Choosing $\kappa=0$ in(3.8), we easily obtain (3.11)-(3.13).

4) Suppose that $\Phi$ is given by (3.8) and $\psi$ verifies (3.7). We know that $\left(M_{t}^{\lambda, \varphi}\right)$ is a non-negative local martingale. We would like to prove that it is in fact a $P_{x}$-martingale.

a) Let $\psi_{n}(y)=\psi(y) 1_{\{y \leq n\}}, \varphi_{n}=\Phi_{n}^{\prime}$, with $\Phi_{n}(y)=1-e^{\lambda y} \int_{y}^{\infty} \psi_{n}(z) e^{-\lambda z} d z$.

Since for any $y \geq x$, we have :

$$
\int_{y}^{\infty} \psi_{n}(z) e^{-\lambda z} d z \leq \int_{y}^{\infty} \psi(z) e^{-\lambda z} d z \leq \int_{x}^{\infty} \psi(z) e^{-\lambda z} d z
$$

then

$$
\begin{aligned}
& \text { - } e^{\lambda X_{t}} \int_{S_{t}}^{\infty} \psi(z) e^{-\lambda z} d z \text { and } e^{\lambda X_{t}} \int_{S_{t}}^{\infty} \psi_{n}(z) e^{-\lambda z} d z \text { are } P_{x} \text {-integrable r.v.'s, } \\
& \text { - } e^{\lambda X_{t}} \int_{S_{t}}^{\infty} \psi_{n}(z) e^{-\lambda z} d z \text { goes to } e^{\lambda X_{t}} \int_{S_{t}}^{\infty} \psi(z) e^{-\lambda z} d z \text {, in } L^{1}\left(\Omega, P_{x}\right) \text {, as } n \rightarrow \infty
\end{aligned}
$$

b) For $u \geq 0$, we have $\sinh (u) \leq e^{u}$, then inequality (3.32) (which will be proved independently later) implies that $E_{x}\left[\psi\left(S_{t}\right) e^{\lambda\left(S_{t}-X_{t}\right)}\right]<\infty$ and

$$
\lim _{n \rightarrow \infty}\left|E_{x}\left[\psi\left(S_{t}\right) e^{\lambda\left(S_{t}-X_{t}\right)}\right]-E_{x}\left[\psi_{n}\left(S_{t}\right) e^{\lambda\left(S_{t}-X_{t}\right)}\right]\right|=\lim _{n \rightarrow \infty} E_{x}\left[\psi\left(S_{t}\right) 1_{\left\{S_{t}>n\right\}} e^{\lambda\left(S_{t}-X_{t}\right)}\right]=0 .
$$

c) Consequently the representation (3.12) implies that $E_{x}\left[M_{t}^{\lambda, \varphi}\right]<\infty$ and $M_{t}^{\lambda, \varphi_{n}}$ goes to $M_{t}^{\lambda, \varphi}$, in $L^{1}\left(\Omega, P_{x}\right)$, as $n \rightarrow \infty$.

It is clear that $\left(M_{t}^{\lambda, \varphi_{n}}\right)$ is a $P_{x}$-martingale. As a result, $\left(M_{t}^{\lambda, \varphi}\right)$ is a $P_{x}$-martingale. 
Remark 3.4 Recall that under $P_{x}^{(-\lambda)},\left(X_{t}\right)$ is a Brownian motion with drift $-\lambda$, started at $x$. Let $\left(\widetilde{M}_{t}\right)$ be the process :

$$
\widetilde{M}_{t}:=M_{t}^{\lambda, \varphi} e^{\left\{\lambda X_{t}+\lambda^{2} t / 2\right\}}=\frac{\psi\left(S_{t}\right)}{2 \lambda}\left(e^{\lambda S_{t}}-e^{\left\{\lambda\left(2 X_{t}-S_{t}\right)\right\}}\right)+e^{2 \lambda X_{t}} \int_{S_{t}}^{\infty} \psi(z) e^{-\lambda z} d z, t \geq 0 .
$$

It is clear that $\left(\widetilde{M}_{t}\right)$ is a $P_{x}^{(-\lambda)}$ martingale.

But $P_{x}^{(-\lambda)}\left(\lim _{t \rightarrow \infty} X_{t}=-\infty\right)=1$, therefore, under $P_{x}^{(-\lambda)}$ :

$$
\lim _{t \rightarrow \infty} \widetilde{M}_{t}=\widetilde{M}_{\infty}:=\frac{\psi\left(S_{\infty}\right)}{2 \lambda} e^{\lambda S_{\infty}} .
$$

Recall that (see e.g. Williams [17]; but this result also follows from Theorem 4.4, 1.):

$$
P_{x}^{(-\lambda)}\left(S_{\infty}>y\right)=P_{0}^{(-\lambda)}\left(S_{\infty}+x>y\right)=e^{-2 \lambda(y-x)}, y \geq x .
$$

This directly implies :

$$
E_{x}^{(-\lambda)}\left[\frac{\psi\left(S_{\infty}\right)}{2 \lambda} e^{\lambda S_{\infty}}\right]=e^{2 \lambda x} \int_{x}^{\infty} \psi(y) e^{-\lambda y} d y<\infty .
$$

Finally, $\left(\widetilde{M}_{t}\right)$ is a non-negative $P_{x}^{(-\lambda)}$ martingale, converging a.s. to $\widetilde{M}_{\infty} \in L^{1}(\Omega)$ and

$$
E_{x}^{(-\lambda)}\left[\widetilde{M}_{0}\right]=(1-\Phi(x)) e^{\lambda x}=e^{2 \lambda x} \int_{x}^{\infty} \psi(y) e^{-\lambda y} d y=E_{x}^{(-\lambda)}\left[\widetilde{M}_{\infty}\right] .
$$

As a result, $\left(\widetilde{M}_{t}\right)$ is a uniformly integrable $P_{x}^{(-\lambda)}$ martingale, and :

$$
\widetilde{M}_{t}=E_{x}^{(-\lambda)}\left[\frac{\psi\left(S_{\infty}\right)}{2 \lambda} e^{\lambda S_{\infty}} \mid \mathcal{F}_{t}\right], t \geq 0 .
$$

This easily implies that $\left(M_{t}^{\lambda, \varphi}\right)$ is a $P_{x}$-martingale. Hence the arguments developed in this remark may be used instead of those in point 4) of the Proof of Proposition 3.S.

Remark 3.5 1. Suppose that $\psi$ verifies the conditions given in 3. of Proposition 3.3 and $\int_{\mathbb{R}} \psi(z) d z=1$. We observe that $M_{t}^{\lambda, \varphi}$, as defined in (3.5), converges as $\lambda \rightarrow 0$, to $M_{t}^{\varphi}$. This leads us to adopt the convention $M_{t}^{0, \varphi}=M_{t}^{\varphi}$.

2. If $\varphi=0$, then $M_{t}^{\lambda, 0}=\cosh \left(\lambda\left(S_{t}-X_{t}\right)\right) e^{-\lambda^{2} t / 2}$.

\subsubsection{Penalization involving the unilateral maximum}

As in sub-section 3.1, $\varphi: \mathbb{R} \mapsto] 0, \infty[$ is a Borel function. We suppose moreover that (3.3) holds. Hence $\varphi$ is actually a probability density function. We denote $\Phi(x)=\int_{-\infty}^{x} \varphi(y) d y$. As we shall see, the martingales involved in the penalization result stated below, belong to the family $\left(M_{t}^{\varphi}\right)$ as defined in Proposition 3.1.

Theorem 3.6 Let $\varphi$ be as above.

1. Let $u \geq 0$ and $x \in \mathbb{R}$. For any $\Gamma_{u}$ in $\mathcal{F}_{u}$, we have:

$$
\lim _{t \rightarrow \infty} \frac{E_{x}\left[1_{\Gamma_{u}} \varphi\left(S_{t}\right)\right]}{E_{x}\left[\varphi\left(S_{t}\right)\right]}=\frac{1}{1-\Phi(x)} E_{x}\left[1_{\Gamma_{u}} M_{u}^{\varphi}\right]
$$

where $\left(M_{u}^{\varphi}\right)_{u \geq 0}$ is the martingale defined in 3.1. 
2. Let $\left(Q_{x}^{\varphi}\right)_{x \in \mathbb{R}}$ be the family of probabilities on $\left(\Omega, \mathcal{F}_{\infty}\right)$ :

$$
Q_{x}^{\varphi}\left(\Gamma_{u}\right)=\frac{1}{1-\Phi(x)} E_{x}\left[1_{\Gamma_{u}} M_{u}^{\varphi}\right], \quad \text { for any } u \geq 0, \text { and } \Gamma_{u} \in \mathcal{F}_{u}
$$

Then, under $Q_{x}^{\varphi}$, the process $\left(X_{t}-x+\int_{0}^{t} \frac{\varphi\left(S_{u}\right)}{M_{u}^{\varphi}} d u ; t \geq 0\right)$ is a Brownian motion, started at 0 .

Remark $3.7\left(M_{t}^{\varphi} ; t \geq 0\right)$ is a $P_{x}$-martingale but it is not uniformly integrable (u.i.) : indeed, $\left(M_{t}^{\varphi} ; t \geq 0\right)$ is a positive martingale, thus it converges a.s. to $M_{\infty}^{\varphi} \geq 0$, as $t \rightarrow \infty$. Let $\left(t_{n}\right)_{n \geq 1}$ be an increasing sequence of times such that $X_{t_{n}}=S_{t_{n}}$, e.g. : $t_{n}=\inf \left\{u \geq 0, X_{u}=n\right\}$ is convenient. Since $M_{t_{n}}^{\varphi}=1-\Phi\left(S_{t_{n}}\right)$, under $P_{x}, M_{t_{n}}^{\varphi}$ goes a.s. to 0 , as $n \rightarrow \infty$. Hence, $M_{\infty}^{\varphi}=0$.

The proof of Theorem 3.6 requires a preliminary result.

Lemma 3.8 Let $x \leq a$ and $\varphi_{0}:\left[a,+\infty\left[\mapsto \mathbb{R}_{+}\right.\right.$such that $\int_{a}^{\infty} \varphi_{0}(u) d u<+\infty$. Then :

$$
\begin{aligned}
& E_{0}\left[\varphi_{0}\left(a \vee\left(x+S_{u}\right)\right)\right]_{u \rightarrow \infty} \sqrt{\frac{2}{\pi u}}\left\{(a-x) \varphi_{0}(a)+\int_{a}^{\infty} \varphi_{0}(y) d y\right\}, \\
& E_{0}\left[\varphi_{0}\left(a \vee\left(x+S_{u}\right)\right)\right] \leq \sqrt{\frac{2}{\pi u}}\left\{(a-x) \varphi_{0}(a)+\int_{a}^{\infty} \varphi_{0}(y) d y\right\}, \\
& E_{0}\left[\varphi_{0}\left(a \vee\left(x+S_{u}\right)\right)\right] \geq \sqrt{\frac{2}{\pi u}} \int_{0}^{\infty} \varphi_{0}(y) e^{-(y-x)^{2} / 2} d y, \quad \text { for any } u \geq 1 .
\end{aligned}
$$

Proof of Lemma 3.8. We have :

$$
E_{0}\left[\varphi_{0}\left(a \vee\left(x+S_{u}\right)\right)\right]=\varphi_{0}(a) P_{0}\left(x+S_{u} \leq a\right)+E_{0}\left[\varphi_{0}\left(x+S_{u}\right) 1_{\left\{x+S_{u} \geq a\right\}}\right]
$$

Recall that under $P_{0}$,

$$
S_{u} \stackrel{(d)}{=}\left|X_{u}\right| \stackrel{(d)}{=} \sqrt{u}\left|X_{1}\right|
$$

Consequently,

$$
E_{0}\left[\varphi_{0}\left(a \vee\left(x+S_{u}\right)\right)\right]=\varphi_{0}(a) \sqrt{\frac{2}{\pi}} \int_{0}^{(a-x) / \sqrt{u}} e^{-z^{2} / 2} d z+\sqrt{\frac{2}{\pi u}} \int_{a}^{\infty} \varphi_{0}(y) e^{-(y-x)^{2} / 2 u} d y .
$$

Then (3.23) and (3.24) follow immediately.

As for (3.25), we have :

$$
E_{0}\left[\varphi\left(a \vee\left(x+S_{u}\right)\right)\right] \geq \sqrt{\frac{2}{\pi u}} \int_{a}^{\infty} \varphi_{0}(y) e^{-(y-x)^{2} / 2 u} d y \geq \sqrt{\frac{2}{\pi u}} \int_{a}^{\infty} \varphi_{0}(y) e^{-(y-x)^{2} / 2} d y,
$$

if $u \geq 1$.

\section{Proof of Theorem 3.6}

1) Let $s \geq 0, x \in \mathbb{R}$ and $\Gamma_{s} \in \mathcal{F}_{s}$ be fixed. We consider $t>s$. Since $S_{t}=S_{s} \vee\left\{X_{s}+\sup _{0 \leq u \leq t-s}\left(X_{u+s}-\right.\right.$ $\left.\left.X_{s}\right)\right\}$, and under $P_{x},\left(X_{u+s}-X_{s} ; u \geq 0\right)$ is a Brownian motion started at 0 ,

$$
E_{x}\left[\varphi\left(S_{t}\right) \mid \mathcal{F}_{s}\right]=\widetilde{\varphi}\left(S_{s}, X_{s} ; t-s\right),
$$


where

$$
\widetilde{\varphi}(a, x ; r)=E_{0}\left[\varphi\left(a \vee\left(x+S_{r}\right)\right)\right]
$$

Lemma 3.8 implies :

$$
E_{x}\left[\varphi\left(S_{t}\right) \mid \mathcal{F}_{s}\right]_{t \rightarrow \infty}^{\sim} \sqrt{\frac{2}{\pi(t-s)}} M_{s}^{\varphi} .
$$

Using Lemma 3.8 with $x=a$, we obtain :

$$
E_{x}\left[\varphi\left(S_{t}\right)\right]=E_{0}\left[\varphi\left(x+S_{t}\right)\right]_{t \rightarrow \infty} \sqrt{\frac{2}{\pi t}}(1-\Phi(x)) .
$$

It is now easy to check (3.21), using the two previous estimates, (3.24), (3.25) and :

$$
\frac{E_{x}\left[1_{\Gamma_{s}} \varphi\left(S_{t}\right)\right]}{E_{x}\left[\varphi\left(S_{t}\right)\right]}=E_{x}\left[1_{\Gamma_{s}} \frac{E_{x}\left[\varphi\left(S_{t}\right) \mid \mathcal{F}_{s}\right]}{E_{x}\left[\varphi\left(S_{t}\right)\right]}\right] .
$$

2) By Proposition 3.1, we know that $\left(\frac{1}{1-\Phi(x)} M_{t}^{\varphi}\right)_{t \geq 0}$ may be written as an exponential martingale. Consequently, point 2) of Theorem 3.6 is a direct consequence of Girsanov's theorem (cf [10], p. 311-313).

We would like to generalize Theorem 3.6, replacing the normalization coefficient $\varphi\left(S_{t}\right)$ by a function of $\left(X_{t}, S_{t}\right)$. As Theorem 3.9 below shows, a good candidate is $\psi\left(S_{t}\right) e^{\lambda\left(S_{t}-X_{t}\right)}$ where $\lambda>0$ and $\psi: \mathbb{R}_{+} \rightarrow \mathbb{R}_{+}$satisfies some conditions.

Theorem 3.9 Let $\lambda>0$ and $\psi: \mathbb{R} \mapsto] 0,+\infty[$ satisfying (3.9). Let $\Phi$ be the function associated with $\psi$ via (3.10) and $\varphi=\Phi^{\prime}$.

1. Let $u>0, x \in \mathbb{R}$ and any $\Gamma_{u}$ in $\mathcal{F}_{u}$. Then :

$$
\lim _{t \rightarrow \infty} \frac{E_{x}\left[1_{\Gamma_{u}} \psi\left(S_{t}\right) e^{\lambda\left(S_{t}-X_{t}\right)}\right]}{E_{x}\left[\psi\left(S_{t}\right) e^{\lambda\left(S_{t}-X_{t}\right)}\right]}=\frac{1}{1-\Phi(x)} E_{x}\left[1_{\Gamma_{u}} M_{u}^{\lambda, \varphi}\right]
$$

where $\left(M_{u}^{\lambda, \varphi}\right)$ is the $P_{x}$-martingale defined in Proposition 3.9 .

2. Let $\left(Q_{x}^{\lambda, \varphi}\right)_{x \in \mathbb{R}}$ be the family of probabilities on $\left(\Omega, \mathcal{F}_{\infty}\right)$ :

$$
Q_{x}^{\lambda, \varphi}\left(\Gamma_{u}\right)=\frac{1}{1-\Phi(x)} E_{x}\left[1_{\Gamma_{u}} M_{u}^{\lambda, \varphi}\right], \quad \text { for any } u \geq 0, \text { and } \Gamma_{u} \in \mathcal{F}_{u}
$$

Then, under $Q_{x}^{\lambda, \varphi}$, the process

$$
\left(X_{t}-x+\lambda \int_{0}^{t} \frac{\varphi\left(S_{u}\right) \cosh \left(\lambda\left(S_{u}-X_{u}\right)\right)+\lambda\left(1-\Phi\left(S_{u}\right)\right) \sinh \left(\lambda\left(S_{u}-X_{u}\right)\right)}{\lambda\left(1-\Phi\left(S_{u}\right)\right) \cosh \left(\lambda\left(S_{u}-X_{u}\right)\right)+\varphi\left(S_{u}\right) \sinh \left(\lambda\left(S_{u}-X_{u}\right)\right)} d u ; t \geq 0\right)
$$

is a Brownian motion, started at 0.

3. In fact, there is the absolute continuity relationship :

$$
Q_{x}^{\lambda, \varphi}=\frac{1}{1-\Phi(x)}\left(\frac{e^{\lambda S_{\infty}} \psi\left(S_{\infty}\right)}{2 \lambda}\right) P_{x}^{(-\lambda)}
$$


Recall (cf 1) of Remark 3.5) that $M^{0, \varphi}=M^{\varphi}, M^{\varphi}$ being the martingale defined in Proposition 3.1. Therefore, Theorem 3.6 may be interpreted as a particular case of Theorem 3.9, since taking formally $\lambda=0$ in (3.27) we recover 3.21). Note that 3. of Theorem 3.9 follows from Remark 3.4.

Our proof of Theorem 3.9 is similar to that of Theorem 3.6. An extension of Lemma 3.8 is required to obtain an equivalent of $E_{0}\left[\psi\left(s \vee\left(x+S_{t}\right)\right) e^{\left.\lambda\left\{s \vee\left(x+S_{t}\right)-x-X_{t}\right)\right\}}\right]$, as $t \rightarrow \infty$. This result is stated below in Lemma 3.10. We observe that the two asymptotic rates of growth are drastically different. This explains why we state two separate results.

Lemma 3.10 Let $s \geq x, s \geq 0$ and $\psi: \mathbb{R} \rightarrow \mathbb{R}$ such that :

$$
\begin{aligned}
& \int_{s}^{\infty}|\psi(z)| e^{-\lambda z} d z<\infty \\
& \rho_{\lambda}(s, x):=\psi(s) \sinh (\lambda(s-x))+\lambda e^{\lambda x} \int_{s}^{\infty} \psi(z) e^{-\lambda z} d z \neq 0 .
\end{aligned}
$$

Then:

$$
E_{0}\left[\psi\left(s \vee\left(x+S_{t}\right)\right) e^{\lambda\left(s_{\vee}\left(x+S_{t}\right)-x-X_{t}\right)}\right]_{t \rightarrow \infty} 2 \rho_{\lambda}(s, x) e^{\lambda^{2} t / 2}
$$

If $\psi \geq 0$, we have :

$$
\begin{aligned}
& E_{0}\left[\psi\left(s \vee\left(x+S_{t}\right)\right) e^{\lambda\left(s_{\vee}\left(x+S_{t}\right)-x-X_{t}\right)}\right] \leq 2 \rho_{\lambda}(s, x)\left(1+\frac{1}{\lambda \sqrt{2 \pi t}}\right) e^{\lambda^{2} t / 2}, \\
& E_{0}\left[\psi\left(s \vee\left(x+S_{t}\right)\right) e^{\lambda\left(s \vee\left(x+S_{t}\right)-x-X_{t}\right)}\right] \geq e^{\lambda^{2} t / 2} \frac{2 \lambda}{\sqrt{2 \pi}} \int_{-\infty}^{x-s} e^{-u^{2} / 2} d u \int_{s}^{\infty} \psi(z) e^{-\lambda z} d z,
\end{aligned}
$$

if $t \geq 1$.

Proof of Lemma 3.10. Let $\Delta$ be the expectation of $\psi\left(s \vee\left(x+S_{t}\right) e^{\left\{\lambda\left(s \vee\left(x+S_{t}\right)-x-X_{t}\right)\right\}}\right.$. We split $\Delta$ in two parts $\Delta_{1}$ and $\Delta_{2}$, corresponding respectively to $\left\{S_{t} \leq s-x\right\}$ and to $\left\{S_{t}>s-x\right\}$ :

$$
\begin{aligned}
& \Delta_{1}=\psi(s) e^{\lambda(s-x)} E_{0}\left[e^{-\lambda X_{t}} 1_{\left\{S_{t}<s-x\right\}}\right] \\
& \Delta_{2}=E_{0}\left[\psi\left(x+S_{t}\right) e^{\lambda\left(S_{t}-X_{t}\right)} 1_{\left\{S_{t}>s-x\right\}}\right] .
\end{aligned}
$$

Recall ([8], section 2.8, p 95) and ([10] section III.3, p 105), that under $P_{0},\left(S_{t}, X_{t}\right)$ is distributed as :

$$
P_{0}\left(S_{t} \in d b, X_{t} \in d a\right)=\frac{2(2 b-a)}{\sqrt{2 \pi t^{3}}} e^{-\frac{(2 b-a)^{2}}{2 t}} 1_{\{a<b, b>0\}} d a d b .
$$

1) Consequently :

$$
\Delta_{1}=\frac{2 \psi(s) e^{\lambda(s-x)}}{\sqrt{2 \pi t^{3}}} \int_{0}^{s-x} d b\left(\int_{-\infty}^{b} e^{-\lambda a}(2 b-a) e^{-\frac{(2 b-a)^{2}}{2 t}} d a\right) .
$$

Setting $c=a-2 b$ in the $a$-integral, we obtain :

$$
\Delta_{1}=\frac{2 \psi(s) e^{\lambda(s-x)}}{\sqrt{2 \pi t}} \int_{0}^{s-x} e^{-2 \lambda b} A(t, b) d b
$$

where $A(t, b)=-\frac{1}{t} \int_{-\infty}^{-b} e^{-\lambda c} c e^{-c^{2} / 2 t} d c$. 
Integrating by parts, we obtain :

$$
A(t, b)=e^{\lambda b} e^{-b^{2} / 2 t}+\lambda \int_{-\infty}^{-b} e^{-\lambda c-c^{2} / 2 t} d c .
$$

Setting $u=\frac{c}{\sqrt{t}}+\lambda \sqrt{t}$ we get :

$$
A(t, b)=e^{\lambda b} e^{-b^{2} / 2 t}+\lambda \sqrt{t} e^{\lambda^{2} t / 2} \int_{-\infty}^{-\frac{b}{\sqrt{t}}+\lambda \sqrt{t}} e^{-u^{2} / 2} d u .
$$

Since $\lim _{t \rightarrow \infty} \int_{-\infty}^{\frac{-b}{\sqrt{t}}+\lambda \sqrt{t}} e^{-u^{2} / 2} d u=\sqrt{2 \pi}$, then $A(t, b)_{t \rightarrow \infty}^{\sim} \lambda \sqrt{2 \pi t} e^{\lambda^{2} t / 2}$, and :

$$
\Delta_{1} \underset{t \rightarrow \infty}{\sim} 2 \psi(s) \sinh (\lambda(s-x)) e^{\lambda^{2} t / 2} .
$$

2) Mimicking the approach developed in 1), we obtain :

$$
\Delta_{2}=\frac{2}{\sqrt{2 \pi t}} \int_{s-x}^{+\infty} \psi(x+b) e^{-\lambda b} A(t, b) d b .
$$

The decomposition (3.35) and the finiteness hypothesis (3.30) imply that :

$$
\Delta_{2} \underset{t \rightarrow \infty}{\sim}\left(2 \lambda \int_{s-x}^{\infty} \psi(x+b) e^{-\lambda b} d b\right) e^{\lambda^{2} t / 2},
$$

3) Suppose $\psi \geq 0$.

a) Applying $\int_{-\infty}^{-\frac{b}{\sqrt{t}}+\lambda \sqrt{t}} e^{-u^{2} / 2} d u \leq \int_{\mathbb{R}} e^{-u^{2} / 2} d u=\sqrt{2 \pi}$, and $\lambda b-\frac{b^{2}}{2 t} \leq \frac{\lambda^{2} t}{2}$ in(3.35) imply that $A(t, b) \leq e^{\lambda^{2} t / 2}(1+\lambda \sqrt{2 \pi t})$ and (3.32).

b) Let $b \in[0, s-x]$ and $t \geq 1$. Then $-\frac{b}{\sqrt{t}}+\lambda \sqrt{t} \geq-\frac{b}{\sqrt{t}} \geq x-s$ and $A(t, b) \geq e^{\lambda^{2} t / 2} \lambda \sqrt{t} \int_{-\infty}^{x-s} e^{-u^{2} / 2} d u$. Using moreover $\Delta \geq \Delta_{2}$ we obtain (3.33).

\section{Proof of Theorem 3.9}

Let $u>0, x \in \mathbb{R}$ and $\Gamma_{u} \in \mathcal{F}_{u}$. Adapting the proof of Theorem 3.6 to our new context, we have :

$$
E_{x}\left[\psi\left(S_{t}\right) e^{\lambda\left(S_{t}-X_{t}\right)} \mid \mathcal{F}_{u}\right]=\widetilde{\psi}\left(S_{u}, X_{u} ; t-u\right),
$$

where :

$$
\widetilde{\psi}(s, x ; r)=E_{0}\left[\psi\left(s \vee\left(x+S_{r}\right)\right) e^{\lambda\left(s \vee\left(x+S_{r}\right)-x-X_{r}\right)}\right] .
$$

Lemma 3.10 gives the rate of increase of $\widetilde{\psi}(s, x ; r), r \rightarrow \infty$, if $\psi \geq 0$ :

$$
\widetilde{\psi}(s, x ; r)_{r \sim \infty}^{\sim} 2 \rho_{\lambda}(s, x) e^{\lambda^{2} r / 2} .
$$

In particular, taking $u=0$, we get :

$$
E_{x}\left[\psi\left(S_{t}\right) e^{\lambda\left(S_{t}-X_{t}\right)}\right]_{t \rightarrow \infty}^{\sim} 2 \rho_{\lambda}(x, x) e^{\lambda^{2} t / 2} .
$$

Moreover if $t>u+1,(3.32)$ and (3.33) imply that :

$$
\frac{\widetilde{\psi}\left(S_{u}, X_{u} ; t-u\right)}{E_{x}\left[\psi\left(S_{t}\right) e^{\lambda\left(S_{t}-X_{t}\right)}\right]} \leq \frac{k}{\lambda} \rho_{\lambda}\left(S_{u}, X_{u}\right) e^{-\lambda u^{2} / 2},
$$


where $k$ is a constant depending only on $x, u, \lambda$.

But $M_{u}^{\lambda, \varphi}=\frac{1}{\lambda} \rho_{\lambda}\left(S_{u}, X_{u}\right) e^{-\lambda u^{2} / 2}$ and $\left(M_{t}^{\lambda, \varphi}\right)$ is a $P_{x}$-martingale; consequently :

$$
\lim _{t \rightarrow \infty} \frac{E_{x}\left[1_{\Gamma_{u}} \psi\left(S_{t}\right) e^{\lambda\left(S_{t}-X_{t}\right)}\right]}{E_{x}\left[\psi\left(S_{t}\right) e^{\lambda\left(S_{t}-X_{t}\right)}\right]}=\frac{1}{\rho_{\lambda}(x, x)} E_{x}\left[1_{\Gamma_{u}} \rho_{\lambda}\left(S_{u}, X_{u}\right) e^{\lambda^{2} u / 2}\right] .
$$

\subsection{Case 2 : the local time at 0}

From Lévy's theorem, under $P_{0},\left(\left(S_{t}-X_{t}, S_{t}\right) ; t \geq 0\right)$ and $\left.\left(\left|X_{t}\right|, L_{t}^{0}\right) ; t \geq 0\right)$ have the same distribution. This implies that $\left(\left|X_{t}\right| \varphi\left(L_{t}^{0}\right)+1-\Phi\left(L_{t}^{0}\right) ; t \geq 0\right)$ is a martingale with respect to the filtration of $\left(\left|X_{t}\right|\right)$, hence with respect to $\left(\mathcal{F}_{t}\right)$, the functions $\varphi$ and $\Phi$ being defined in Proposition 3.1. These processes are particular cases of more general martingales :

Proposition 3.11 Let $h^{+}, h^{-}: \mathbb{R}_{+} \longrightarrow \mathbb{R}_{+}$, be bounded, Borel functions, and define :

$$
H(l)=\frac{1}{2} \int_{0}^{l}\left(h^{+}(u)+h^{-}(u)\right) d u, \quad l \geq 0 .
$$

1. Then :

$$
M_{t}^{h^{+}, h^{-}}=1-H\left(L_{t}^{0}\right)+X_{t}^{+} h^{+}\left(L_{t}^{0}\right)+X_{t}^{-} h^{-}\left(L_{t}^{0}\right),
$$

is a $P_{0}$-martingale. Moreover $M_{0}^{h^{+}, h^{-}}=1 P_{0}$ a.s., and:

$$
M_{t}^{h^{+}, h^{-}}=1+\int_{0}^{t}\left(1_{\left\{X_{s}>0\right\}} h^{+}\left(L_{s}^{0}\right)-1_{\left\{X_{s}<0\right\}} h^{-}\left(L_{s}^{0}\right)\right) d X_{s}
$$

2. If moreover :

$$
\frac{1}{2} \int_{0}^{\infty}\left(h^{+}(u)+h^{-}(u)\right) d u=1
$$

and $H(l)<1$, for any $l>0$, then $M_{t}^{h^{+}, h^{-}}>0$ and $M_{t}^{h^{+}, h^{-}}=\mathcal{E}\left(J^{h^{+}, h^{-}}\right)_{t}$, where :

$$
J_{t}^{h^{+}, h^{-}}=\frac{1_{\left\{X_{t}>0\right\}} h^{+}\left(L_{t}^{0}\right)-1_{\left\{X_{t}<0\right\}} h^{-}\left(L_{t}^{0}\right)}{1-H\left(L_{t}^{0}\right)+X_{t}^{+} h^{+}\left(L_{t}^{0}\right)+X_{t}^{-} h^{-}\left(L_{t}^{0}\right)} .
$$

The martingales $\left(M_{t}^{h^{+}, h^{-}}\right)$featured in (3.37) and (3.38) have already been used, e.g., in [7]. Both statements of Propositions 3.1, 3.11 are also found in (10], Chapter VI, "first order calculus") and are particular cases of application of the balayage formula. More precisely, formula (3.38) may be generalized as follows: if $\left(h_{s}^{+} ; s \geq 0\right)$ and $\left(h_{s}^{-} ; s \geq 0\right)$ are bounded predictable processes, and if $g_{t}=\sup \left\{s \leq t ; X_{s}=0\right\}$, then :

$$
X_{t}^{+} h_{g_{t}}^{+}+X_{t}^{-} h_{g_{t}}^{-}-\frac{1}{2} \int_{0}^{t}\left(h_{s}^{+}+h_{s}^{-}\right) d L_{s}^{0}=\int_{0}^{t}\left(h_{g_{s}}^{+} 1_{\left\{X_{s}>0\right\}}-h_{g_{s}}^{-} 1_{\left\{X_{s}<0\right\}}\right) d X_{s} .
$$

Remark 3.12 1. Recall that in Proposition 3.3 we have introduced the family of martingales $\left(M_{t}^{\lambda, \varphi}\right)$; this should not induce any confusion with the family $\left(M_{t}^{h^{+}, h^{-}}\right)$, since the two parameters indexing the first (resp. second) family are respectively $(\lambda, \varphi)$ and $\left(h^{+}, h^{-}\right)$, and belong to quite different sets. 
2. In this section we restrict ourselves to $P_{0}$. Thus $\left(X_{t}\right)$ is a Brownian motion started at 0 . It would also be possible to work under $P_{x}$, replacing $\left(M_{t}^{h^{+}, h^{-}}\right)$by $\left(1-H\left(L_{t}^{x}\right)+\left(X_{t}-x\right)^{+} h^{+}\left(L_{t}^{x}\right)+\right.$ $\left.\left(X_{t}-x\right)^{-} h^{-}\left(L_{t}^{x}\right) ; t \geq 0\right)$. However, for simplicity we only deal with $x=0$.

We now investigate penalizations involving the local time at 0 of $X$.

Theorem 3.13 Let $\left(h^{+}, h^{-}\right)$and $\left(M_{t}^{h^{+}, h^{-}}\right)$be the functions and the martingale defined in Proposition 3.11. We suppose that (3.39) holds.

1. Let $s \geq 0$ and $\Gamma_{s} \in \mathcal{F}_{s}$. Then :

$$
\lim _{t \rightarrow \infty} \frac{E_{0}\left[1_{\Gamma_{s}}\left(h^{+}\left(L_{t}^{0}\right) 1_{\left\{X_{t}>0\right\}}+h^{-}\left(L_{t}^{0}\right) 1_{\left\{X_{t}<0\right\}}\right)\right]}{E_{0}\left[h^{+}\left(L_{t}^{0}\right) 1_{\left\{X_{t}<0\right\}}+h^{-}\left(L_{t}^{0}\right) 1_{\left\{X_{t}<0\right\}}\right]}=E_{0}\left[1_{\Gamma_{s}} M_{s}^{h^{+}, h^{-}}\right] .
$$

2. Let $Q_{0}^{h^{+}, h^{-}}$be the probability measure on $\left(\Omega, \mathcal{F}_{\infty}\right)$ satisfying :

$$
Q_{0}^{h^{+}, h^{-}}\left(\Gamma_{s}\right)=E_{0}\left[1_{\Gamma_{s}} M_{s}^{h^{+}, h^{-}}\right],
$$

for any $s \geq 0$ and $\Gamma_{s} \in \mathcal{F}_{s}$.

Then under $Q_{0}^{h^{+}, h^{-}}$, the process :

$$
\left(X_{t}-\int_{0}^{t} \frac{h^{+}\left(L_{s}^{0}\right) 1_{\left\{X_{s}>0\right\}}-h^{-}\left(L_{s}^{0}\right) 1_{\left\{X_{s}<0\right\}}}{M_{s}^{h^{+}, h^{-}}} d s ; t \geq 0\right)
$$

is a Brownian motion.

Remark 3.14 As observed in Remark 3.7, the $P_{0}$-martingale $\left(M_{t}^{h^{+}, h^{-}}\right)$is not uniformly integrable, in fact, if $\tau_{l}=\inf \left\{s>0, L_{s}^{0}>l\right\}$, then : $M_{\tau_{l}}^{h^{+}, h^{-}}$goes a.s. to 0 as $l \rightarrow \infty$.

The proof of part 1) of Theorem 3.13 is based on the following estimate.

Lemma 3.15 Let $f: \mathbb{R}_{+} \longrightarrow \mathbb{R}_{+}$be Borel and locally bounded such that $\int_{0}^{\infty} f(s) d s<\infty$. Let $a \geq 0$ and $x \in \mathbb{R}$, then

$$
\begin{aligned}
& E_{x}\left[f\left(a+L_{t}^{0}\right) 1_{\left\{X_{t}>0\right\}}\right]_{t \rightarrow \infty} f(a) \sqrt{\frac{2}{\pi t}} x^{+}+\frac{1}{\sqrt{2 \pi t}} \int_{a}^{\infty} f(s) d s, \\
& E_{x}\left[f\left(a+L_{t}^{0}\right) 1_{\left\{X_{t}<0\right\}}\right]_{t \rightarrow \infty} f(a) \sqrt{\frac{2}{\pi t}} x^{-}+\frac{1}{\sqrt{2 \pi t}} \int_{a}^{\infty} f(s) d s .
\end{aligned}
$$

Proof of Lemma 3.15. Since under $P_{x},\left(-X_{t}\right)_{t \geq 0}$ is distributed as $\left(X_{t}\right)$ under $\left.P_{-x}, 3.44\right)$ is a direct consequence of (3.43).

To prove (3.43), we recall the well-known result :

$$
P_{x}\left(T_{a} \in d t\right)=\frac{|x-a|}{\sqrt{2 \pi t^{3}}} \exp \left\{-\frac{(x-a)^{2}}{2 t}\right\} 1_{\{t>0\}} d t .
$$

On the set $\left\{T_{0}>t\right\}$, we have, $P_{x}$ a.s. :

$$
f\left(a+L_{t}^{0}\right) 1_{\left\{X_{t}>0\right\}}=\left\{\begin{array}{cl}
0 & \text { if } x<0 \\
f(a) & \text { otherwise }
\end{array}\right.
$$


Then

$$
E_{x}\left[f\left(a+L_{t}^{0}\right) 1_{\left\{X_{t}>0\right\}}\right]=f(a) P_{x}\left(T_{0}>t\right) 1_{\{x>0\}}+\Delta_{t},
$$

where

$$
\Delta_{t}=E_{x}\left[f\left(a+L_{t}^{0}\right) 1_{\left\{X_{t}>0, t \geq T_{0}\right\}}\right]
$$

Using (3.26) we have :

$$
P_{x}\left(T_{0}>t\right)=P_{0}\left(T_{|x|}>t\right)=P_{0}\left(S_{t}<|x|\right)=P_{0}\left(\left|X_{1}\right|<\frac{|x|}{\sqrt{t}}\right) .
$$

Therefore :

$$
P_{x}\left(T_{0}>t\right)_{t \rightarrow \tilde{+} \infty} \sqrt{\frac{2}{\pi t}}|x| .
$$

To compute $\Delta_{t}$, we use the strong Markov property at time $T_{0}$, and we get :

$$
\Delta_{t}=E_{x}\left[1_{\left\{t \geq T_{0}\right\}} g\left(t-T_{0}\right)\right],
$$

with $g(r)=E_{0}\left[f\left(a+L_{r}^{0}\right) 1_{\left\{X_{r}>0\right\}}\right]$.

Since under $P_{0},\left(-X_{u}\right)$ and $\left(X_{u}\right)$ have the same distribution,

$$
g(r)=\frac{1}{2} E_{0}\left[f\left(a+L_{r}^{0}\right)\right]=\frac{1}{2} E_{0}\left[f\left(a+S_{r}\right)\right],
$$

the last equality being a consequence of Lévy's theorem.

Applying Lemma 3.8 (with $s=x=a, u=r$ and $\varphi_{0}=f$ ), we obtain :

$$
g(r)_{r \rightarrow \infty} \frac{1}{\sqrt{2 \pi r}} \int_{a}^{\infty} f(s) d s .
$$

Consequently, $\Delta_{t} \underset{t \rightarrow \infty}{\sim} \frac{1}{\sqrt{2 \pi t}} \int_{a}^{\infty} f(s) d s$. (3.43) follows immediately.

The remainder of the proof of Theorem 3.13 is left to the reader, since it is similar to the proofs of Theorems 3.6 and 3.9 .

\subsection{Case 3 : the maximum, the infimum and the local time.}

For simplicity we restrict ourselves to $P_{0}$. The family of martingales playing a central role in this section may be new. This family is related to martingales of the type $\left(M_{t}^{h^{+}, h^{-}}\right)$. More precisely, let :

$$
h^{+}(l)=\frac{1}{a} e^{c l}, h^{-}(l)=\frac{1}{b} e^{c l},
$$

with $c=\frac{1}{2}\left(\frac{1}{a}+\frac{1}{b}\right), a>0$ and $b>0$. Then $H(l)=e^{c l}-1$ and

$$
M_{t}^{h^{+}, h^{-}}=2-\left(1-\frac{X_{t}^{+}}{a}-\frac{X_{t}^{-}}{b}\right) e^{c L_{t}^{0}}
$$

Since $X_{t}^{+} X_{t}^{-}=0$, then :

$$
M_{t}^{h^{+}, h^{-}}=2-\left(1-\frac{X_{t}^{+}}{a}\right)\left(1-\frac{X_{t}^{-}}{b}\right) e^{c L_{t}^{0}} .
$$


In particular,

$$
2-M_{t \wedge T_{a} \wedge T_{-b}}^{h^{+}, h^{-}}=\left(1-\frac{X_{t}^{+}}{a}\right)\left(1-\frac{X_{t}^{-}}{b}\right) e^{c L_{t}^{0}} 1_{\left\{t \leq T_{a} \wedge T_{-b}\right\}},
$$

is a $P_{0}$-martingale.

Integrating this identity with respect to some positive measure $\nu(d a, d b)$ we obtain the following result.

Proposition 3.16 Let $\nu$ be a probability measure on $\mathbb{R}_{+} \times \mathbb{R}_{+}$, whose support is included in $[\alpha,+\infty[\times[\alpha,+\infty[$, for some $\alpha>0$, and $\left(M_{t}^{\nu}\right)$ be the process :

$$
\begin{aligned}
M_{t}^{\nu} & =\int_{\mathbb{R}_{+} \times \mathbb{R}_{+}}\left(1-\frac{X_{t}^{+}}{a}\right)\left(1-\frac{X_{t}^{-}}{b}\right) \exp \left\{\frac{1}{2}\left(\frac{1}{a}+\frac{1}{b}\right) L_{t}^{0}\right\} 1_{\left\{t \leq T_{a} \wedge T_{-b}\right\}} \nu(d a, d b) \\
& =\int_{\mathbb{R}_{+} \times \mathbb{R}_{+}}\left(1-\frac{X_{t}^{+}}{a}\right)\left(1-\frac{X_{t}^{-}}{b}\right) \exp \left\{\frac{1}{2}\left(\frac{1}{a}+\frac{1}{b}\right) L_{t}^{0}\right\} 1_{\left\{S_{t} \leq a, I_{t} \leq b\right\}} \nu(d a, d b) .
\end{aligned}
$$

Then, under $P_{0},\left(M_{t}^{\nu} ; t \geq 0\right)$ is a positive martingale, $M_{0}^{\nu}=1, M_{t}^{\nu}=\mathcal{E}\left(J^{\nu}\right)_{t}$ with :

$$
J_{t}^{\nu}=\frac{1}{M_{t}^{\nu}} \int_{\mathbb{R}_{+} \times \mathbb{R}_{+}}\left(-\frac{1}{a} 1_{\left\{X_{t}>0\right\}}+\frac{1}{b} 1_{\left\{X_{t}<0\right\}}\right) \exp \left\{\frac{1}{2}\left(\frac{1}{a}+\frac{1}{b}\right) L_{t}^{0}\right\} 1_{\left\{S_{t} \leq a, I_{t} \leq b\right\}} \nu(d a, d b) .
$$

Remark 3.17 1. Obviously if we take for $\nu$ the Dirac measure at $(a, b)$, for some $a>0, b>0$, then we recover (3.5).

2. We may write $\left(M_{t}^{\nu}\right)$ as follows :

$$
M_{t}^{\nu}=F\left(S_{t}, I_{t}, L_{t}^{0}\right)-X_{t}^{+} F^{+}\left(S_{t}, I_{t}, L_{t}^{0}\right)-X_{t}^{-} F^{-}\left(S_{t}, I_{t}, L_{t}^{0}\right),
$$

with

$$
\begin{aligned}
& F^{+}(s, i, l)=\int_{\mathbb{R}_{+}^{2}} 1_{\{s \leq a, i \leq b\}} \frac{1}{a} \exp \left\{\frac{1}{2}\left(\frac{1}{a}+\frac{1}{b}\right) l\right\} \nu(d a, d b), \\
& F^{-}(s, i, l)=\int_{\mathbb{R}_{+}^{2}} 1_{\{s \leq a, i \leq b\}} \frac{1}{b} \exp \left\{\frac{1}{2}\left(\frac{1}{a}+\frac{1}{b}\right) l\right\} \nu(d a, d b), \\
& F(s, i, l)=\int_{\mathbb{R}_{+}^{2}} 1_{\{s \leq a, i \leq b\}} \exp \left\{\frac{1}{2}\left(\frac{1}{a}+\frac{1}{b}\right) l\right\} \nu(d a, d b) .
\end{aligned}
$$

If $\nu$ is assumed to have a continuous density function, then it follows that :

$$
\begin{aligned}
& \frac{1}{2}\left(F^{+}+F^{-}\right)=\frac{\partial F}{\partial l}, \\
& s \frac{\partial F^{+}}{\partial s}(s, i, l)=\frac{\partial F}{\partial s}(s, i, l) ; i \frac{\partial F^{-}}{\partial i}(s, i, l)=\frac{\partial F}{\partial i}(s, i, l) .
\end{aligned}
$$

3. Suppose that $\nu$ is a p.m. with support included in the diagonal, then $\left(M_{t}^{\nu}\right)$ coincides with $M_{t}^{\nu *}$ where

$$
M_{t}^{\nu_{*}}:=\int_{X_{t}^{*}}^{\infty}\left(1-\frac{\left|X_{t}\right|}{a}\right) e^{L_{t}^{0} / a} \nu_{*}(d a)
$$


$\nu_{*}$ being, in this case, a p.m. on $[\alpha,+\infty[, \alpha>0$. Consequently, relations 3.5. - 3.50) become :

$$
M_{t}^{\nu_{*}}=F\left(X_{t}^{*}, L_{t}^{0}\right)-\left|X_{t}\right| F^{*}\left(X_{t}^{*}, L_{t}^{0}\right),
$$

with :

$$
F(x, l)=\int_{x}^{\infty} e^{l / a} \nu_{*}(d a), \quad F^{*}(x, l)=\int_{x}^{\infty} \frac{1}{a} e^{l / a} \nu_{*}(d a)
$$

Moreover $M_{t}^{\nu_{*}}=\mathcal{E}\left(J^{\nu_{*}}\right)_{t}$ with :

$$
J_{t}^{\nu_{*}}=-\frac{\operatorname{sgn}\left(X_{t}\right)}{M_{t}^{\nu_{*}}} \int_{X_{t}^{*}}^{\infty} \frac{1}{a} e^{L_{t}^{0} / a} \nu_{*}(d a) .
$$

4. Sometimes, we shall consider similar martingales involving only the one-sided maximum $\left(S_{t}\right)$ and $\left(L_{t}^{0}\right)$; hence, we shall use, instead of (3.50):

$$
2-M_{t \wedge T_{a}}^{h^{+}, 0}=\left(1-\frac{X_{t \wedge T_{a}}^{+}}{a}\right) e^{L_{t \wedge T_{a}}^{0} / 2 a}
$$

where $h^{+}(l)=\frac{1}{a} e^{l / 2 a}$.

Integrating over $\left[\alpha, \infty\left[\right.\right.$, with respect to a p.m. $\nu_{+}$on $[\alpha, \infty[$, leads to :

$$
M_{t}^{\nu_{+}}=\int_{S_{t}}^{\infty}\left(1-\frac{X_{t}^{+}}{a}\right) e^{L_{t}^{0} / 2 a} \nu_{+}(d a)
$$

As previously $\left(M_{t}^{\nu_{+}}\right)$is a $P_{0}$-martingale, such that $M_{0}^{\nu_{+}}=1$, and it may be written as :

$$
M_{t}^{\nu_{+}}=F\left(S_{t}, L_{t}^{0}\right)-X_{t}^{+} F^{+}\left(S_{t}, L_{t}^{0}\right)
$$

with $F(s, l)=\int_{s}^{\infty} e^{l / 2 a} \nu_{+}(d a)$ and $F^{+}(s, l)=\int_{s}^{\infty} \frac{1}{a} e^{l / 2 a} \nu_{+}(d a)$.

We now deal with penalizations involving jointly the maximum, the minimum and the local time of $X$. Recall (see Proposition 3.16) that $\nu$ is a p.m. on $\left[\alpha,+\infty\left[\times\left[\alpha,+\infty\left[\right.\right.\right.\right.$ for some $\alpha>0$ and $\left(M_{t}^{\nu}\right)$ is the positive martingale defined by (3.51).

Theorem 3.18 Let $A_{\nu}$ be the function:

$$
A_{\nu}(s, i, l)=\int_{\mathbb{R}_{+}^{2}} e^{\frac{1}{2}\left(\frac{1}{a}+\frac{1}{b}\right) l} 1_{\{s \leq a, i \leq b\}} \nu(d a, d b), s, i, l \geq 0 .
$$

1. Let $u \geq 0$ and $\Gamma_{u} \in \mathcal{F}_{u}$. Then :

$$
\lim _{t \rightarrow \infty} \frac{E_{0}\left[1_{\Gamma_{u}} A_{\nu}\left(S_{t}, I_{t}, L_{t}^{0}\right)\right]}{E_{0}\left[A_{\nu}\left(S_{t}, I_{t}, L_{t}^{0}\right)\right]}=E_{0}\left[1_{\Gamma_{u}} M_{u}^{\nu}\right] .
$$

2. Let $Q_{0}^{\nu}$ be the p.m. on $\left(\Omega, \mathcal{F}_{\infty}\right)$ which satisfies :

$$
Q_{0}^{\nu}\left(\Gamma_{u}\right)=E_{0}\left[1_{\Gamma_{u}} M_{u}^{\nu}\right]
$$

for any $u \geq 0$ and $\Gamma_{u} \in \mathcal{F}_{u}$.

The process $\left(X_{t}-\int_{0}^{t} \frac{J_{s}^{\nu}}{M_{s}^{\nu}} d s ; t \geq 0\right)$ is a $Q_{0}^{\nu}$-Brownian motion, where $\left(J_{t}^{\nu}\right)$ is defined by 3.59). 
The asymptotic result (3.64) is based on the following rather striking result.

Lemma 3.19 Let $a>0, b>0$ and $c=\left(\frac{1}{a}+\frac{1}{b}\right)$. Then

$$
\begin{aligned}
& \lim _{t \rightarrow \infty} E_{0}\left[e^{c L_{t}^{0}} 1_{\left\{t<T_{-b} \wedge T_{a}\right\}}\right]=\frac{3}{2}, \\
& \sup _{t \geq 0} E_{0}\left[e^{c L_{t}^{0}} 1_{\left\{t<T_{-b} \wedge T_{a}\right\}}\right]<+\infty .
\end{aligned}
$$

It would be possible to directly prove Lemma 3.19, but the proof is technical ${ }^{1}$. Instead, we provide a short proof based on a disintegration of the p.m. $Q_{0}^{\nu}$ (see Theorem 5.3 for details). This is why we have postponed this proof to Section 5 (which is devoted to such disintegrations), just after Theorem 5.3 .

\section{Proof of Theorem 3.18 .}

Let $u \geq 0$ and $\Gamma_{u} \in \mathcal{F}_{u}$ be fixed, $t>u$ and $\delta\left(\Gamma_{u}, t\right)=E_{0}\left[1_{\Gamma_{u}} A_{\nu}\left(S_{t}, I_{t}, L_{t}^{0}\right)\right]$.

By (3.63), we have :

$$
A_{\nu}\left(S_{t}, I_{t}, L_{t}^{0}\right)=\int_{\mathbb{R}_{+}^{2}} e^{\frac{1}{2}\left(\frac{1}{a}+\frac{1}{b}\right) L_{t}^{0}} 1_{\left\{T_{a}>t, T_{-b}>t\right\}} \nu(d a, d b) .
$$

Applying the Markov property at time $u$, we obtain :

$$
\delta\left(\Gamma_{u}, t\right)=\int_{\mathbb{R}_{+}^{2}} E_{0}\left[1_{\Gamma_{u}} 1_{\left\{T_{a} \wedge T_{-b}>u\right\}} e^{c L_{u}^{0}} \pi_{1}\left(a, b, X_{u}, t-u\right)\right] \nu(d a, d b) .
$$

where $c=\frac{1}{2}\left(\frac{1}{a}+\frac{1}{b}\right)$, and

$$
\pi_{1}(a, b, x, r)=E_{x}\left[1_{\left\{T_{a} \wedge T_{-b}>r\right\}} e^{c L_{r}^{0}}\right] ;-b \leq x \leq a, r \geq 0 .
$$

Let us start with $x>0$.

Since under $P_{x},\left\{T_{a} \wedge T_{-b}>r\right\}=\left\{T_{a} \wedge T_{0}>r\right\} \cup\left\{T_{0}<T_{a}, T_{a} \wedge T_{-b}>r\right\}$ we have :

$$
\begin{aligned}
& \pi_{1}(a, b, x, r)=P_{x}\left(T_{a} \wedge T_{0}>r\right)+\pi_{2}(a, b, x, r), \\
& \pi_{2}(a, b, x, r)=E_{x}\left[1_{\left\{T_{0}<T_{a}, T_{a} \wedge T_{-b}>r\right\}} e^{c L_{r}^{0}}\right] .
\end{aligned}
$$

Applying the strong Markov property at time $T_{0}$, we get :

$$
\pi_{2}(a, b, x, r)=E_{x}\left[1_{\left\{T_{0}<T_{a}, T_{0}<r\right\}} \pi_{3}\left(a, b, r-T_{0}\right)\right]
$$

with :

$$
\pi_{3}\left(a, b, r^{\prime}\right)=E_{0}\left[e^{c L_{r^{\prime}}^{0}} 1_{\left\{r^{\prime}<T_{-b} \wedge T_{a}\right\}}\right] .
$$

Lemma 3.19 implies that $\pi_{2}$ is bounded and $\pi_{2}(a, b, x, r)$ converges to $\frac{3}{2} \frac{a-x}{a}$, as $r \rightarrow+\infty$.

As for $P_{x}\left(T_{a} \wedge T_{0}>r\right)$, it is clear that this probability goes to 0 , as $r \rightarrow \infty$.

A similar approach may be developed for $x<0$. Inequality (3.67) implies that $\pi_{1}$ is bounded ; consequently, for $\Gamma_{u} \in \mathcal{F}_{u}$ :

$$
\lim _{t \rightarrow \infty} \delta\left(\Gamma_{u}, t\right)=\frac{3}{2} E_{0}\left[1_{\Gamma_{u}} \int_{\mathbb{R}_{+}^{2}} e^{c L_{u}^{0}} 1_{\left\{T_{a} \wedge T_{-b}>u\right\}}\left\{\frac{a-X_{u}}{a} 1_{\left\{X_{u} \geq 0\right\}}+\frac{b+X_{u}}{b} 1_{\left\{X_{u}<0\right\}}\right\} \nu(d a, d b)\right] .
$$

The result follows because $\frac{a-X_{u}}{a} 1_{\left\{X_{u} \geq 0\right\}}+\frac{b+X_{u}}{b} 1_{\left\{X_{u}<0\right\}}$ is equal to $\left(1-\frac{X_{u}^{+}}{a}\right)\left(1-\frac{X_{u}^{-}}{b}\right)$ and $E_{0}\left[M_{u}^{\nu}\right]=1$.

\footnotetext{
${ }^{1}$ We thank F. Petit who helped us with such a proof
} 


\subsection{Case 4 : the down-crossings.}

Let $a$ and $b$ be two fixed numbers, $a<b$. To describe the down-crossings of $\left(X_{t}\right)$, from level $b$ to level $a$, it is convenient to introduce the sequence of stopping times defined inductively as follows :

$$
\begin{aligned}
& \sigma_{1}=\inf \left\{t \geq 0, X_{t}>b\right\}, \\
& \sigma_{2}=\inf \left\{t \geq \sigma_{1}, X_{t}<a\right\},
\end{aligned}
$$

and

$$
\begin{aligned}
& \sigma_{2 n+1}=\inf \left\{t \geq \sigma_{2 n} ; X_{t}>b\right\}, \\
& \sigma_{2 n+2}=\inf \left\{t \geq \sigma_{2 n+1} ; X_{t}<a\right\} .
\end{aligned}
$$

Let $D_{t}$ be the number of down-crossings from level $b$ to level $a$, up to time $t$ :

$$
D_{t}=\sum_{n \geq 1} 1_{\left\{\sigma_{2 n} \leq t\right\}} ; t \geq 0 .
$$

We observe that :

$$
\sigma_{2 D_{s}}=\sup \left\{n \geq 1 ; \sigma_{2 n} \leq s\right\},
$$

with the convention $\sup \{\emptyset\}=0$ and $\sigma_{0}=0$.

The events $\left\{\sigma_{2 D_{s}}+T_{b} \circ \theta_{\sigma_{2 D_{s}}}>s\right\}$ and $\left\{\sigma_{2 D_{s}}+T_{b} \circ \theta_{\sigma_{2 D_{s}}} \leq s\right\}$ will play a central role below. If $\left\{\sigma_{2 n} \leq s<\sigma_{2 n+2}\right\}$, the first (resp. second) event reduces to $\left\{\sigma_{2 n} \leq s<\sigma_{2 n+1}\right\}$ (resp. $\left\{\sigma_{2 n+1} \leq s<\right.$ $\left.\left.\sigma_{2 n+2}\right\}\right)$.

Proposition 3.20 Let $(G(n))_{n \geq 0}$ be a sequence of real numbers, and $\left(M_{t}^{\downarrow}, G\right)$ be the process :

$$
\begin{aligned}
M_{t}^{\downarrow, G}= & 1_{\left\{\sigma_{2 D_{t}}+T_{b} \circ \theta_{\sigma_{2 D_{t}}}>t\right\}}\left(\frac{G\left(D_{t}\right)}{2}\left(1+\frac{b-X_{t}}{b-a}\right)+\frac{G\left(1+D_{t}\right)}{2}\left(\frac{X_{t}-a}{b-a}\right)\right) \\
& +1_{\left\{\sigma_{2 D_{t}}+T_{b} \circ \theta_{\sigma_{2 D_{t}}} \leq t\right\}}\left(\frac{G\left(1+D_{t}\right)}{2}\left(1+\frac{b-X_{t}}{b-a}\right)+\frac{G\left(D_{t}\right)}{2}\left(\frac{X_{t}-a}{b-a}\right)\right) .
\end{aligned}
$$

1. Then $\left(M_{t}^{\downarrow}, G\right)$ is a continuous $P_{x}$-local martingale,

$$
\begin{aligned}
& M_{t}^{\downarrow, G}= \sum_{n \geq 0}\left\{1_{\left[\sigma_{2 n}, \sigma_{2 n+1}[\right.}(t)\left(\frac{G(n)}{2}\left(1+\frac{b-X_{t}}{b-a}\right)+\frac{G(1+n)}{2}\left(\frac{X_{t}-a}{b-a}\right)\right)\right. \\
&+1_{\left[\sigma_{2 n+1}, \sigma_{2 n+2}[\right.}(t)\left(\frac{G(1+n)}{2}\left(1+\frac{b-X_{t}}{b-a}\right)+\frac{G(n)}{2}\left(\frac{X_{t}-a}{b-a}\right)\right\}, \\
& M_{t}^{\downarrow, G}=\quad M_{0}^{\downarrow, G}+\frac{1}{b-a} \int_{0}^{t}\left(1_{\left\{\sigma_{2 D_{s}}+T_{b} \circ \theta_{\sigma_{2 D_{s}}}>s\right\}}-1_{\left\{\sigma_{2 D_{s}}+T_{b} \circ \theta_{\sigma_{2 D_{s}}}<s\right\}}\right) \\
& \times\left(\frac{G\left(1+D_{s}\right)-G\left(D_{s}\right)}{2}\right) d X_{s},
\end{aligned}
$$

and under $P_{x}$ :

$$
M_{0}^{\downarrow}, G= \begin{cases}\frac{1}{2(b-a)}(G(0)(2 b-a-x)+G(1)(x-a)) & \text { if } x \leq b, \\ \frac{1}{2(b-a)}(G(0)(x-a)+G(1)(2 b-a-x)) & \text { otherwise. }\end{cases}
$$


2. Suppose moreover that $(G(n))_{n \geq 0}$ is a decreasing sequence of positive real numbers such that $G(0)=1$ and $\lim _{n \rightarrow \infty} G(n)=0$. Then $M_{t}^{\downarrow, G}>0$ and $M_{t}^{\downarrow, G}=M_{0}^{\downarrow, G} \mathcal{E}\left(J^{\downarrow, G}\right)_{t}$ with

$$
\begin{gathered}
J_{t}^{\downarrow, G}=\left(G\left(1+D_{t}\right)-G\left(D_{t}\right)\right)\left\{\frac{1}{G\left(D_{t}\right)\left(2 b-X_{t}-a\right)+G\left(1+D_{t}\right)\left(X_{t}-a\right)} 1_{\left\{\sigma_{2 D_{t}}+T_{b} \circ \theta_{2 D_{t}}>t\right\}}\right. \\
\left.-\frac{1}{G\left(1+D_{t}\right)\left(2 b-X_{t}-a\right)+G\left(D_{t}\right)\left(X_{t}-a\right)} 1_{\left\{\sigma_{2 D_{t}}+T_{b} \circ \theta_{2 D_{t}} \leq t\right\}}\right\} .
\end{gathered}
$$

Proof 1) A priori, $\left(M_{t}^{\downarrow, G}\right)$ is a right continuous process, and may only jump at times $\sigma_{n}$. However, it is easy to check that $M_{\sigma_{n-}}^{\downarrow, G}=M_{\sigma_{n}}^{\downarrow, G}$, therefore $\left(M_{t}^{\downarrow, G} ; t \geq 0\right)$ is continuous. (A posteriori, this is "automatic", as soon as we know that $\left(M_{t}^{\downarrow}, G\right)$ is a local martingale in the Brownian filtration.) We have :

$$
M_{t}^{\downarrow, G}=\frac{G\left(D_{t}\right)+G\left(1+D_{t}\right)}{2}+\left(\frac{b-X_{t}}{b-a}\right)\left(\frac{G\left(D_{t}\right)-G\left(D_{t}+1\right)}{2}\right) \operatorname{sign}\left(\sigma_{2 D_{t}}+T_{b} \circ \theta_{\sigma_{2 D_{t}}}-t\right) .
$$

Since $t \rightarrow D_{t}$ and $t \rightarrow \operatorname{sgn}\left(\sigma_{2 D_{t}}+T_{b} \circ \theta_{\sigma_{2 D_{t}}}-t\right)$ are piecewise constant processes, and $\left(M_{t}^{\downarrow, G}\right)$ is continuous, applying Ito's formula leads to $(3.76)$. Hence $\left(M_{t}^{\downarrow, G}\right)$ is a continuous local martingale. Relation (3.79) implies that:

$$
\left|M_{t}^{\downarrow, G}\right| \leq \sup _{n \geq 0}|G(n)|\left(1+\frac{|b|+X_{t}^{*}}{b-a}\right)
$$

Consequently, $\left(M_{t}^{\downarrow}, G ; t \geq 0\right)$ is a martingale.

2) Suppose that $G(n)>0, G(0)=1$, and $n \rightarrow G(n)$ decreases to 0 . If $\sigma_{2 D_{t}}+T_{b} \circ \theta_{\sigma_{2 D_{t}}}>t$, then $b-X_{t} \geq 0$ and formula (3.79) gives :

$$
M_{t}^{\downarrow, G} \geq \frac{G\left(D_{t}\right)+G\left(1+D_{t}\right)}{2}>0 .
$$

If $\sigma_{2 D_{t}}+\sigma_{1} \circ \theta_{\sigma_{2 D_{t}}} \leq t$, we first modify (3.74) as follows :

$$
M_{t}^{\downarrow, G}=\left(\frac{X_{t}-a}{b-a}\right) \frac{G\left(D_{t}\right)-G\left(1+D_{t}\right)}{2}+G\left(1+D_{t}\right) .
$$

But $X_{t} \geq a$, consequently $M_{t}^{\downarrow, G} \geq G\left(1+D_{t}\right)>0$.

Finally $M_{t}^{\downarrow, G}>0$.

To end the proof of Proposition 3.20 we observe that (3.78) is a direct consequence of (3.76) and (3.74).

We state below our last theorem concerning penalization results. Here the underlying process is the number $D_{t}$ of down-crossings from $b$ to $a$. Recall that the sequence of stopping times $\left(\sigma_{n}\right)_{n \geq 1}$ associated with $\left(D_{t}\right)$ is defined through (3.68)-(3.71). Let $G: \mathbb{N} \rightarrow \mathbb{R}_{+}$, be a decreasing function, such that:

$$
G(0)=1, \quad G(\infty):=\lim _{n \rightarrow \infty} G(n)=0 .
$$

Let $(\Delta G(n))_{n \geq 0}$ be the sequence of positive numbers :

$$
\Delta G(n)=G(n)-G(n+1), \quad n \geq 0 .
$$


Theorem 3.21 Let $G: \mathbb{N} \rightarrow \mathbb{R}_{+}$as above.

1. Let $s \geq 0, \Gamma_{s} \in \mathcal{F}_{s}$, then :

$$
\lim _{t \rightarrow \infty} \frac{E_{0}\left[1_{\Gamma_{s}} \Delta G\left(D_{t}\right)\right]}{E_{0}\left[\Delta G\left(D_{t}\right)\right]}=\frac{1}{M_{0}^{\downarrow, G}} E_{0}\left[1_{\Gamma_{s}} M_{s}^{\downarrow, G}\right]
$$

where $\left(M_{t}^{\downarrow, G}\right)$ is the positive martingale defined in Proposition 3.20.

2. Let $Q_{0}^{\downarrow, G}$ be the p.m. defined on $\left(\Omega, \mathcal{F}_{\infty}\right)$ :

$$
Q_{0}^{\downarrow, G}\left(\Gamma_{s}\right)=\frac{1}{M_{0}^{\downarrow, G}} E_{0}\left[1_{\Gamma_{s}} M_{s}^{\downarrow, G}\right],
$$

for any $s \geq 0$ and $\Gamma_{s} \in \mathcal{F}_{s}$.

Then the process :

$$
\begin{aligned}
& X_{t}-\int_{0}^{t}\left(G\left(1+D_{s}\right)-G\left(D_{s}\right)\right)\left\{\frac{1}{G\left(D_{s}\right)\left(2 b-X_{s}-a\right)+G\left(1+D_{s}\right)\left(X_{s}-a\right)}\right. \\
\times & \left.1_{\left\{\sigma_{2 D_{s}}+T_{b} \circ \theta_{2 D_{s}}>s\right\}}-\frac{1}{G\left(1+D_{s}\right)\left(2 b-X_{s}-a\right)+G\left(D_{s}\right)\left(X_{s}-a\right)} 1_{\left\{\sigma_{2 D_{s}}+T_{b} \circ \theta_{2 D_{s}} \leq s\right\}}\right\} d s
\end{aligned}
$$

is a $Q_{0}^{\downarrow, G}$ Brownian motion.

The proof of Theorem 3.21 is based on the following asymptotic estimate.

Lemma 3.22 Let $x \in \mathbb{R}$ and $(H(n))_{n \geq 0}$ be a sequence of positive real numbers satisfying: $\sum_{n \geq 0} H(n)<$ $\infty$. Then :

$$
\lim _{t \rightarrow \infty} \sqrt{t} E_{x}\left[H\left(D_{t}\right)\right]=2(b-a) \sqrt{\frac{2}{\pi}}\left\{\sum_{n \geq 1} H(n)+H(0)\left(\frac{1}{2}+\frac{|x-b|}{2(b-a)}\right)\right\} .
$$

Proof. On the one hand we observe that the definition (3.72) of $D_{t}$ implies $\left\{D_{t} \geq n\right\}=\left\{\sigma_{2 n} \leq t\right\}$. On the other hand, under $P_{x}, \sigma_{1}, \sigma_{2}-\sigma_{1}, \cdots, \sigma_{2 n}-\sigma_{2 n-1}$ are independent, $\sigma_{1}$ (resp. $\sigma_{i+1}-\sigma_{i}$ ) is distributed as $T_{|b-x|}\left(\right.$ resp. $\left.T_{b-a}\right)$ under $P_{0}$. Consequently :

$$
P_{x}\left(D_{t} \geq n\right)=P_{0}\left(T_{|b-x|+(2 n-1)(b-a)} \leq t\right) .
$$

Since the probability on the right hand-side equals $P_{0}\left(S_{t} \geq|b-x|+(2 n-1)(b-a)\right)$, the scaling property (3.26) implies :

$$
P_{x}\left(D_{t} \geq n\right)=P_{0}\left(n \leq \frac{\sqrt{t}\left|X_{1}\right|+b-a-|b-x|}{2(b-a)}\right) ; n \geq 1 .
$$

$D_{t}$ being a $\mathbb{N}$-valued r.v., we have :

$$
E_{x}\left[H\left(D_{t}\right)\right]=H(0)+\sum_{n \geq 1}(H(n)-H(n-1)) P_{x}\left(D_{t} \geq n\right)
$$

Let $\xi_{t}:=\frac{\sqrt{t}\left|X_{1}\right|+b-a-|b-x|}{2(b-a)}$. Using Fubini's theorem and (3.86) we get

$$
\begin{aligned}
E_{x}\left[H\left(D_{t}\right)\right] & =H(0)-E_{0}\left[1_{\left\{\xi_{t} \geq 1\right\}} \sum_{n=1}^{\left[\xi_{t}\right]}(H(n)-H(n-1))\right] \\
& =H(0) P_{0}\left(\xi_{t}<1\right)+E_{0}\left[H\left(\left[\xi_{t}\right]\right) 1_{\left\{\xi_{t} \geq 1\right\}}\right] .
\end{aligned}
$$


where $[a]$ denotes the integer part of $a$.

It is easy to compute the last expectation :

$$
E_{0}\left[H\left(\left[\xi_{t}\right]\right) 1_{\left\{\xi_{t} \geq 1\right\}}\right]=\sqrt{\frac{2}{\pi}} \int_{0}^{\infty} H\left(\left[\frac{z \sqrt{t}+b-a-\mid b-x]}{2(b-a)}\right]\right) e^{-z^{2} / 2} 1_{\left\{\frac{z \sqrt{t}+b-a-|b-x|}{2(b-a)}>1\right\}} d z .
$$

Setting $y=\frac{z \sqrt{t}+b-a-|b-x|}{2(b-a)}$, we have :

$$
E_{0}\left[H\left(\left[\xi_{t}\right]\right) 1_{\left\{\xi_{t} \geq 1\right\}}\right]=2(b-a) \sqrt{\frac{2}{\pi t}} \int_{1}^{\infty} H([y]) \exp \left\{-\frac{(2(b-a) y-b+a+|b-x|)^{2}}{2 t}\right\} d y .
$$

Since $\sum_{n \geq 1} H(n)<\infty$

$$
\lim _{t \rightarrow \infty}\left(\sqrt{t} E_{0}\left[H\left(\left[\xi_{t}\right]\right) 1_{\left\{\xi_{t} \geq 1\right\}}\right]\right)=2(b-a) \sqrt{\frac{2}{\pi}}\left(\sum_{n \geq 1} H(n)\right) .
$$

It remains to study $P_{0}\left(\xi_{t}<1\right)$. We have successively :

$$
P_{0}\left(\xi_{t}<1\right)=P_{0}\left(\left|X_{1}\right|<\frac{b-a+|b-x|}{\sqrt{t}}\right),
$$

hence :

$$
\lim _{t \rightarrow \infty} \sqrt{t} P\left(\xi_{t}<1\right)=(b-a+|b-x|) \sqrt{\frac{2}{\pi}} .
$$

This ends the proof of (3.85).

\section{Proof of Theorem 3.21}

Let $s \geq 0, \Gamma_{s} \in \mathcal{F}_{s}$ be fixed, $t>s$ and consider the quantity $E_{0}\left[1_{\Gamma_{s}} \Delta G\left(D_{t}\right)\right]$.

Let us introduce the events :

$$
\Sigma_{1}=\left\{\sigma_{2 D_{s}}+T_{b} \circ \theta_{\sigma_{2 D_{s}}}>s\right\}, \Sigma_{2}=\Sigma_{1}^{c}=\left\{\sigma_{2 D_{s}}+T_{b} \circ \theta_{\sigma_{2 D_{s}}} \leq s\right\},
$$

and decompose the above expectation accordingly.

1) On $\Sigma_{1}$ :

$$
D_{t}=D_{t-s} \circ \theta_{s}+D_{s} .
$$

Applying Lemma 3.22 with $x=X_{s}, H(n)=\Delta G\left(n+D_{s}\right)$, after conditioning by $\mathcal{F}_{s}$, we obtain :

$$
E_{0}\left[1_{\Gamma_{s} \cap \Sigma_{1}} \Delta G\left(D_{t}\right)\right]_{t \rightarrow \infty}^{\sim} 2(b-a) \sqrt{\frac{2}{\pi}} E_{0}\left[1_{\Gamma_{s} \cap \Sigma_{1}} J_{1}\right] \frac{1}{\sqrt{t-s}},
$$

where $J_{1}=\Delta G\left(D_{s}\right)\left(\frac{1}{2}+\frac{\left|X_{s}-b\right|}{2(b-a)}\right)+\sum_{n \geq 1} \Delta G\left(D_{s}+n\right)$.

Since on $\Sigma_{1}, X_{s} \leq b$, it is easy to check that :

$$
J_{1}=\frac{G\left(D_{s}\right)}{2}\left(1+\frac{b-X_{s}}{b-a}\right)+\frac{G\left(1+D_{s}\right)}{2}\left(\frac{X_{s}-a}{b-a}\right) .
$$

As a result :

$$
E_{0}\left[1_{\Gamma_{s} \cap \Sigma_{1}} \Delta G\left(D_{t}\right)\right]_{t \rightarrow \infty}^{\sim}\left\{2(b-a) \sqrt{\frac{2}{\pi}} E_{0}\left[1_{\Gamma_{s} \cap \Sigma_{1}} M_{s}^{\downarrow}, G\right]\right\} \frac{1}{\sqrt{t}}
$$


2) We decompose $\Sigma_{2}$ in two disjoint events : $\Sigma_{2}=\Sigma_{2}^{\prime} \cup \Sigma_{3}$ where $\Sigma_{2}^{\prime}=\Sigma_{2} \cap\left\{X_{s}>b\right\}$ and $\Sigma_{3}=$ $\Sigma_{2} \cap\left\{a<X_{s} \leq b\right\}$.

On $\Sigma_{2} \cap\left\{X_{s}>b\right\},(3.87)$ holds and as previously :

$$
E_{x}\left[1_{\Gamma_{s} \cap \Sigma_{2} \cap\left\{X_{s}>b\right\}} \Delta G\left(D_{t}\right)\right]_{t \rightarrow \infty}^{\sim}\left(2(b-a) \sqrt{\frac{2}{\pi}} E_{x}\left[1_{\Gamma_{s} \cap \Sigma_{2} \cap\left\{X_{s}>b\right\}} J_{1}\right]\right) \frac{1}{\sqrt{t-s}} .
$$

Since $X_{s}>b, J_{1}=\frac{G\left(1+D_{s}\right)}{2}\left(1+\frac{b-X_{s}}{b-a}\right)+\frac{G\left(D_{s}\right)}{2}\left(\frac{X_{s}-a}{b-a}\right)$ and

$$
E_{0}\left[1_{\Gamma_{s} \cap \Sigma_{2} \cap\left\{X_{s}>b\right\}} \Delta G\left(D_{t}\right)\right]_{t \rightarrow \infty}^{\sim}\left\{2(b-a) \sqrt{\frac{2}{\pi}} E_{0}\left[1_{\Gamma_{s} \cap \Sigma_{2} \cap\left\{X_{s}>b\right\}} M_{s}^{\downarrow, G}\right]\right\} \frac{1}{\sqrt{t}} .
$$

3) We split $\Sigma_{3}$ in three disjoint subsets : $\Sigma_{3}=\Sigma_{4} \cup \Sigma_{5} \cup \Sigma_{6}$, with

$$
\begin{aligned}
& \Sigma_{4}=\Sigma_{3} \cap\left\{s+T_{a} \circ \theta_{s}<s+T_{b} \circ \theta_{s}<t\right\}, \\
& \Sigma_{5}=\Sigma_{3} \cap\left\{s+T_{b} \circ \theta_{s}<s+T_{a} \circ \theta_{s}<t\right\}, \\
& \Sigma_{6}=\Sigma_{4} \cap\left\{\left(s+T_{b} \circ \theta_{s}\right) \wedge\left(s+T_{a} \circ \theta_{s}\right) \geq t\right\} .
\end{aligned}
$$

We set $U_{a}=s+T_{a} \circ \theta_{s}$ and $U_{b}=s+T_{b} \circ \theta_{s}$.

a) On $\Sigma_{4}$, we have :

$$
D_{t}=D_{U_{a}}+D_{t-U_{a}} \circ \theta_{U_{a}}=1+D_{s}+D_{t-U_{a}} \circ \theta_{U_{a}} .
$$

Applying the strong Markov property at time $U_{a}$, together with Lemma 3.22, we obtain on $\Sigma_{4}$ :

$$
\lim _{t \rightarrow \infty} \sqrt{t} E_{0}\left[\Delta G\left(D_{t}\right) \mid \mathcal{F}_{U_{a}}\right]=2(b-a) \sqrt{\frac{2}{\pi}} G\left(D_{U_{a}}\right) .
$$

But $D_{U_{a}}=1+D_{s}$; hence, taking the conditional expectation with respect to $\mathcal{F}_{s}$, we get :

$$
\lim _{t \rightarrow \infty} \sqrt{t} E_{0}\left[1_{\Gamma_{s} \cap \Sigma_{4}} \Delta G\left(D_{t}\right)\right]=2(b-a) \sqrt{\frac{2}{\pi}} E_{0}\left[1_{\Gamma_{s} \cap \Sigma_{3}} \frac{b-X_{s}}{b-a} G\left(1+D_{s}\right)\right] .
$$

b) On $\Sigma_{5}, D_{t}=D_{U_{b}}+D_{t-U_{b}} \circ \theta_{U_{b}}=D_{s}+D_{t-U_{b}} \circ \theta_{U_{b}}$. We proceed as previously; we obtain successively :

$$
\begin{gathered}
\lim _{t \rightarrow \infty} \sqrt{t} E_{0}\left[1_{\Sigma_{5}} \Delta G\left(D_{t}\right) \mid \mathcal{F}_{U_{b}}\right]=2(b-a) \sqrt{\frac{2}{\pi}} \frac{G\left(D_{U_{b}}\right)+G\left(D_{U_{b}}+1\right)}{2} 1_{\left\{U_{b}<U_{a}\right\}}, \\
\lim _{t \rightarrow \infty} \sqrt{t} E_{0}\left[1_{\Gamma_{s} \cap \Sigma_{5}} \Delta G\left(D_{t}\right)\right]=2(b-a) \sqrt{\frac{2}{\pi}} E_{0}\left[1_{\Gamma_{s} \cap \Sigma_{3}} \frac{X_{s}-a}{b-a} \frac{G\left(D_{s}\right)+G\left(1+D_{s}\right)}{2}\right] .
\end{gathered}
$$

c) We claim that $\Sigma_{6}$ does not contribute to the limit since

$$
\begin{aligned}
P_{0}\left(\Sigma_{6} \mid \mathcal{F}_{s}\right) & =1_{\Sigma_{3}} P_{X_{s}}\left(T_{a} \wedge T_{b}>t-s\right) \leq 1_{\Sigma_{3}} P_{\frac{a+b}{2}}\left(T_{a} \wedge T_{b}>t-s\right) \\
& \leq 1_{\Sigma_{3}} e^{-\lambda(t-s)},
\end{aligned}
$$

for some $\lambda>0$.

Finally :

$$
E_{0}\left[1_{\Gamma_{s} \cap \Sigma_{2} \cap\left\{X_{s} \leq b\right\}} \Delta G\left(D_{t}\right)\right]_{t \rightarrow \infty}^{\sim}\left\{2(b-a) \sqrt{\frac{2}{\pi}} E_{0}\left[1_{\Gamma_{s} \cap \Sigma_{2} \cap\left\{X_{s} \leq b\right\}} M_{s}^{\downarrow, G}\right]\right\} \frac{1}{\sqrt{t}} .
$$

4) Consequently, thanks to the previous steps we have :

$$
E_{0}\left[1_{\Gamma_{s}} \Delta G\left(D_{t}\right)\right]_{t \rightarrow \infty}^{\sim}\left\{2(b-a) \sqrt{\frac{2}{\pi}} E_{0}\left[1_{\Gamma_{s}} M_{s}^{\downarrow}, G\right]\right\} \frac{1}{\sqrt{t}} .
$$

This implies 3.82).

Point 2. of Theorem 3.21 is a direct consequence of Proposition 3.20 . 


\section{Study of the $Q$-processes. An approach via enlargements of filtrations}

In sections 3.1-3.4 we have obtained penalization principles involving unilateral maximum, unilateral maximum and time, local time at 0 , maximum + minimum + local time at 0 and finally down-crossings. In each case, a positive and continuous martingale $\left(M_{t} ; t \geq 0\right)$ appears naturally. This allows to define $Q_{x}$ on $\left(\Omega, \mathcal{F}_{\infty}\right)$ via : $Q_{x}\left(\Gamma_{t}\right)=E_{x}\left[1_{\Gamma_{t}} M_{t}\right]$, for any $\Gamma_{t} \in \mathcal{F}_{t}, t \geq 0$.

As said in the Introduction, $Q_{x}$ is a well-defined p.m. on $\left(\Omega, \mathcal{F}_{\infty}\right)$.

This leads us to describe the law of $\left(X_{t}\right)_{t \geq 0}$ under $Q_{x}, x \in \mathbb{R}$.

We are able to handle the two first cases recalled previously via a general approach, which is developed in subsection 4.1. Unfortunately the other cases cannot be handled in this way, and we have to study them one by one. Our approach then is based on enlargements of filtrations. However the schemes of proof are similar in all cases, consequently we only discuss Case 1 in details. Concerning the other cases, we only state the results and sketch their proofs giving the key points without detailed arguments.

\subsection{Some general results and their applications}

We consider here a general setting, where we are given a filtered probability space $\left(\Omega, \mathcal{F},\left(\mathcal{F}_{t}\right)_{t \geq 0}, \mathbb{P}\right)$, a strictly positive continuous martingale $\left(M_{t} ; t \geq 0\right)$, with respect to $\left(\left(\mathcal{F}_{t}\right)_{t \geq 0}, \mathbb{P}\right)$, starting at 1 at $t=0$, and a second probability $\mathbb{Q}$ on $\left(\Omega, \mathcal{F}_{\infty}\right)$ such that :

$$
\mathbb{Q}\left(\Gamma_{t}\right)=\mathbb{E}\left[1_{\Gamma_{t}} M_{t}\right], \Gamma_{t} \in \mathcal{F}_{t}, t \geq 0 .
$$

We define :

$$
\underline{M}_{t}=\inf _{0 \leq s \leq t} M_{s}
$$

The following discussion is inspired from [2]

Proposition 4.1 1. The process $\left(Y_{t}=\frac{M_{t}}{\underline{M}_{t}}-1, t \geq 0\right)$ is a non-negative, continuous, local $\mathbb{P}$ submartingale and $\mathbb{P}\left(Y_{0}=0\right)=1$.

2. Let $\left(l_{t} ; t \geq 0\right)$ be the non-decreasing, continuous process, such that $l_{0}=0$ and $\left(Y_{t}-l_{t}\right)_{t \geq 0}$ is a continuous local martingale. Then the support of $d l_{t}$ is included in $\left\{t \geq 0 ; Y_{t}=0\right\}$ and

$$
\underline{M}_{t}=e^{-l_{t}} ; t \geq 0
$$

Proof. Using Ito's formula we have :

$$
d Y_{t}=\frac{d M_{t}}{\underline{M}_{t}}-\frac{M_{t}}{\underline{M}_{t}^{2}} d \underline{M}_{t} .
$$

Consequently $\left(Y_{t}\right)$ is a local submartingale and :

$$
d l_{t}=-\frac{M_{t}}{\underline{M}_{t}^{2}} d \underline{M}_{t}=-\frac{1}{\underline{M}_{t}} d \underline{M}_{t} .
$$

This implies that $\operatorname{supp}\left(d l_{t}\right)=\operatorname{supp}\left(d M_{t}\right)$.

Integrating (4.4) over $[0, t]$ leads to (4.3).

To go further, we need an additional assumption; i.e.,we assume :

$$
M_{\infty}=0 \quad \mathbb{P} \text { a.s. }
$$

It is clear that $M_{\infty}=0$ iff $\underline{M}_{\infty}=0$. From (4.3) this condition is equivalent to $l_{\infty}=+\infty$. 
Theorem 4.2 Let $\mathbb{Q}$ be the p.m. defined by (4.1).

1. $\underline{M}_{\infty}$ is a $\mathbb{Q}$-finite r.v. with uniform distribution on $[0,1]$.

2. Let $g:=\sup \left\{t \geq 0, M_{t}=\underline{M}_{\infty}\right\}$ (with the convention $\left.\sup \emptyset=0\right)$. Then $\mathbb{Q}(0<g<\infty)=1$. Let

$$
Z_{t}=\mathbb{Q}\left(g>t \mid \mathcal{F}_{t}\right) ; t \geq 0 .
$$

Then

(a) $Z_{t}=\underline{M}_{t} / M_{t}$,

(b) $\left(Z_{t}\right)$ is a positive, $\mathbb{Q}$-supermartingale with additive decomposition:

$$
Z_{t}=1-\int_{0}^{t} \frac{M_{u}}{M_{u}^{2}} d \widetilde{M}_{u}+\ln \left(\underline{M}_{t}\right)
$$

where $\widetilde{M}_{t}=M_{t}-\int_{0}^{t} \frac{d<M>_{u}}{M_{u}}$ is the martingale part of $\left(M_{t}\right)$ under $\mathbb{Q}$.

Proof of Theorem 4.2 i) Let us determine the distribution function of $\underline{M}_{\infty}$ under $\mathbb{Q}$. Let $t>0$ and $0<c<1$. We have :

$$
\mathbb{Q}\left(\underline{M}_{t}<c\right)=\mathbb{Q}(\sigma(c)<t)=\mathbb{E}_{x}\left[1_{\{\sigma(c)<t\}} M_{t}\right],
$$

where $\sigma(c)=\inf \left\{u \geq 0, M_{u}<c\right\}$.

Applying Doob's optional stopping theorem we obtain :

$$
\mathbb{Q}\left(\underline{M}_{t}<c\right)=\mathbb{E}\left[1_{\{\sigma(c)<t\}} M_{\sigma(c)}\right]=c \mathbb{P}(\sigma(c)<t) .
$$

Taking $t \rightarrow \infty$, we obtain : $\mathbb{Q}\left(\underline{M}_{\infty}<c\right)=c$.

ii) Let us compute $E_{\mathbb{Q}}\left[g>t \mid \mathcal{F}_{t}\right]$, where $t>0$ is fixed. Let $\Gamma_{t}$ be in $\mathcal{F}_{t}$. We have :

$$
\mathbb{Q}\left(\Gamma_{t} \cap\{g>t\}\right)=\mathbb{Q}\left(\Gamma_{t} \cap\left\{\sigma_{t}^{\prime}<\infty\right\}\right),
$$

where $\sigma_{t}^{\prime}=\inf \left\{s>t ; M_{s} \leq \underline{M}_{t}\right\}$.

Consequently :

$$
\mathbb{Q}\left(\Gamma_{t} \cap\{g>t\}\right)=\lim _{n \rightarrow \infty} \delta_{n},
$$

with $\delta_{n}=\mathbb{Q}\left(\Gamma_{t} \cap\left\{\sigma_{t}^{\prime} \leq t+n\right\}\right)$.

Using the same technique as in step i), we have successively :

$$
\begin{aligned}
\delta_{n} & =\mathbb{E}\left[1_{\Gamma_{t} \cap\left\{\sigma_{t}^{\prime} \leq t+n\right\}} M_{t+n}\right]=\mathbb{E}\left[1_{\Gamma_{t} \cap\left\{\sigma_{t}^{\prime} \leq t+n\right\}} M_{\sigma_{t}^{\prime}}\right] \\
& =\mathbb{E}\left[1_{\Gamma_{t} \cap\left\{\sigma_{t}^{\prime} \leq t+n\right\}} \underline{M}_{t}\right] .
\end{aligned}
$$

Letting $n \rightarrow \infty$, we get :

$$
\mathbb{Q}\left(\Gamma_{t} \cap\{g>t\}\right)=\mathbb{E}\left[1_{\Gamma_{t}} \underline{M}_{t}\right]=E_{\mathbb{Q}}\left[1_{\Gamma_{t}} \underline{M}_{t} / M_{t}\right] .
$$

This proves that $Z_{t}=\underline{M}_{t} / M_{t}$ (under $\mathbb{Q}$ ).

Equivalently to (4.8), we have :

$$
\mathbb{Q}\left(\Gamma_{t} \cap\{0<g \leq t\}\right)=\mathbb{Q}\left(\Gamma_{t}\right)-\mathbb{E}\left[1_{\Gamma_{t}} \underline{M}_{t}\right],
$$

from which it follows that $\mathbb{Q}(0<g<\infty)=1$.

iii) Since $t \rightarrow 1_{\{g>t\}}$ is non-increasing, then $\left(Z_{t}\right)$ is a $\mathbb{Q}$-supermartingale. Applying Ito's formula we get :

$$
d Z_{t}=\frac{d \underline{M}_{t}}{M_{t}}-\frac{M_{t}}{M_{t}^{2}} d M_{t}+\frac{\underline{M}_{t}}{M_{t}^{3}} d<M>_{t} .
$$

The decomposition (4.7) is a direct consequence of Girsanov's theorem. 
Remark 4.3 We write $: \mathbb{P} \longrightarrow \mathbb{Q}$ in case the pair $(\mathbb{P}, \mathbb{Q})$ satisfies the absolute continuity relationship $(4.1)$, as well as (4.5). We claim that this relation is symmetric, i.e. if $\mathbb{P} \longrightarrow \mathbb{Q}$, then $\mathbb{Q} \longrightarrow \mathbb{P}$. Indeed, let $N_{t}=1 / M_{t}, t \geq 0$. It is clear that $\left(N_{t}\right)$ is a positive and continuous $\mathbb{Q}$ martingale, starting at 1 . To prove the claim, we have to check that $\lim _{t \rightarrow \infty} N_{t}=0, \mathbb{Q}$ a.s.

Let $A>1$ be a real number and $\sigma_{A}=\inf \left\{t \geq 0 ; M_{t} \geq A\right\}$. Recall (10], Ex 3.12, Chap. II) that $\sup M_{t}$ is distributed as $1 / U$ where $U$ is uniformly distributed on $[0,1]$.

$t \geq 0$

Let $t$ be a fixed real number. We have :

$$
\mathbb{Q}\left(\sigma_{A}<t\right)=\mathbb{E}\left[1_{\left\{\sigma_{A}<t\right\}} M_{t}\right]=\mathbb{E}\left[1_{\left\{\sigma_{A}<t\right\}} M_{\sigma_{A}}\right]=A \mathbb{P}\left(\sigma_{A}<t\right) .
$$

Taking $t \rightarrow \infty$, we get :

$$
\mathbb{Q}\left(\sigma_{A}<\infty\right)=A \mathbb{P}\left(\sigma_{A}<\infty\right)=A \mathbb{P}\left(\sup _{t \geq 0} M_{t}>A\right)=A \frac{1}{A}=1 .
$$

Since $A$ is arbitrary, this implies that $\sup _{t \geq 0} M_{t}=+\infty, \mathbb{Q}$ a.s. Consequently, under $\mathbb{Q}, \lim _{t \rightarrow \infty} N_{t}=0$.

Applying Remark 4.3 with Theorem 4.2 we obtain the following.

Theorem 4.4 Let $\left(N_{t} ; t \geq 0\right)$ be a positive and continuous $\mathbb{Q}$ martingale such that under $\mathbb{Q}: N_{0}=1$ and $\lim _{t \rightarrow \infty} N_{t}=0$.

1. $\sup _{t \geq 0} N_{t} \stackrel{(d)}{=} 1 / U$, where $U$ is uniformly distributed on $[0,1]$.

2. If $g=\sup \left\{t \geq 0 ; N_{t}=\sup _{u \geq 0} N_{u}\right\}$, then: $\mathbb{Q}(0<g<\infty)=1$. Let $Z_{t}=\mathbb{Q}\left(g>t \mid \mathcal{F}_{t}\right), t \geq 0$. Then

(a) $Z_{t}=\frac{N_{t}}{\bar{N}_{t}}$, where $\bar{N}_{t}:=\sup _{0 \leq u \leq t} N_{u}$.

(b) $\left(Z_{t}\right)$ is a positive, $\mathbb{Q}$-supermartingale, with Doob-Meyer decomposition: $Z_{t}=M_{t}^{\prime}-\ln \left(\bar{N}_{t}\right)$, where $\left(M_{t}^{\prime}\right)$ denotes a $\mathbb{Q}$ martingale.

We now make the further assumption that $\mathbb{P}=P_{x}$ is the Wiener measure on the canonical space $\mathcal{C}\left(\mathbb{R}_{+}, \mathbb{R}\right)$, and $P_{x}\left(X_{0}=x\right)=1$. We shall also write $Q_{x}$ for $\mathbb{Q}$, in this particular case. We now gather a number of complements to our previous general results in this particular instance :

1. Since $\left(M_{t}\right)_{t \geq 0}$ is a $P_{x}$-martingale and $M_{0}=1$, then the representation theorem of Brownian martingales ([10], section V.3, p.192) implies that $\left(M_{t}\right)$ may be written as :

$$
M_{t}=1+\int_{0}^{t} m_{s} d X_{s}
$$

where $\left(m_{s}\right)$ is a predictable process, such that for any $t \geq 0, \int_{0}^{t} m_{s}^{2} d s<+\infty$ a.s.

2. The process $\left(\beta_{t}=X_{t}-\int_{0}^{t} \frac{m_{u}}{M_{u}} d u ; t \geq 0\right)$ is a $Q_{x}$-Brownian motion started at $x$.

Remark 4.5 For the sake of efficiency, we now use the technique of progressive enlargement of filtrations (see for instance [0] or [18]). Let $\left(\mathcal{G}_{t}\right)$ be the smallest filtration containing $\left(\mathcal{F}_{t}\right)$ and such that $g$ is a $\left(\mathcal{G}_{t}\right)$-stopping time. Then:

$$
\beta_{t}=\widetilde{\beta}_{t}+\int_{0}^{t \wedge g} \frac{d<Z, \beta>_{u}}{Z_{u}}-\int_{t \wedge g}^{t} \frac{d<Z, \beta>_{u}}{1-Z_{u}} ; t \geq 0,
$$


where $\left(\widetilde{\beta}_{t} ; t \geq 0\right)$ is a $\left(\left(\mathcal{G}_{t}\right), Q_{x}\right)$-Brownian motion started at $x$.

Since $Z_{t}=\underline{M}_{t} / M_{t}$, applying directly (4.7) leads to :

$$
\beta_{t}=\widetilde{\beta}_{t}-\int_{0}^{t \wedge g} \frac{m_{u}}{M_{u}} d u+\int_{t \wedge g}^{t} \frac{\underline{M}_{u} m_{u}}{M_{u}\left(M_{u}-\underline{M}_{u}\right)} d u .
$$

Recall that $X_{t}=\beta_{t}+\int_{0}^{t} \frac{m_{u}}{M_{u}} d u$, consequently:

$$
X_{t}=\widetilde{\beta}_{t}+\int_{t \wedge g}^{t} \frac{m_{u}}{M_{u}-\underline{M}_{u}} d u
$$

In particular $X_{t}=\widetilde{\beta}_{t}$ for any $t \leq g$. Let $\sigma(y)=\inf \left\{t \geq 0, M_{t}<y\right\}$, for any $0<y<1$. Then conditionally on $\underline{M}_{\infty}=y$, the process $\left(X_{t} ; 0 \leq t \leq g\right)$ is distributed as a Brownian motion started at $x$ and considered up to its first hitting time of level $y$.

Going back to (4.10), we note that, for $u>g$, the drift term equals

$$
\frac{m_{u}}{M_{u}-\underline{M}_{\infty}} \cdot
$$

and, in general, $\left(X_{t}\right)$ is not a diffusion, except in particular cases, see below Theorems 4.8 and 4.8 .

Our first application of Theorem 4.2 concerns $Q_{x}^{\varphi}$. Recall that

$$
Q_{x}^{\varphi}\left(\Gamma_{t}\right)=\frac{1}{1-\Phi(x)} E_{x}\left[1_{\Gamma_{t}} M_{t}^{\varphi}\right]
$$

for any $t>0$ and $\Gamma_{t} \in \mathcal{F}_{t}$, where $M_{t}^{\varphi}=\left(S_{t}-X_{t}\right) \varphi\left(S_{t}\right)+1-\Phi\left(S_{t}\right)$, and $\varphi$ satisfies the conditions given in Proposition 3.1 and (3.3).

Theorem 4.6 1. Under $Q_{x}^{\varphi}$, the r.v. $S_{\infty}$ is positive, finite and admits $\frac{\varphi(y)}{1-\Phi(x)} 1_{\{y \geq x\}}$ as a density function.

2. Let $g=\sup \left\{u \geq 0 ; X_{u}=S_{\infty}\right\}$. Then $Q_{x}^{\varphi}(0<g<\infty)=1$, and under $Q_{x}^{\varphi}$ :

(a) the processes $\left(X_{u} ; u \leq g\right)$ and $\left(X_{g}-X_{u+g} ; u \geq 0\right)$ are independent,

(b) $\left(X_{g}-X_{u+g} ; u \geq 0\right)$ is distributed as a three dimensional Bessel process started at 0 ,

(c) conditionally on $S_{\infty}=z>x,\left(X_{u} ; u \leq g\right)$ is distributed as a Brownian motion started at $x$ and stopped at its first hitting time of $z$.

Proof. a) Let $(a, b)$ be a maximal interval of excursion of $\left(X_{t}\right)$ below its unilateral supremum $\left(S_{t}\right)$. Since for any $u \in] a, b\left[, S_{u}-X_{u}>0\right.$ and $S_{u}=S_{a}=S_{b}$, then $\inf _{0 \leq u \leq t} M_{u}^{\varphi}=1-\Phi\left(S_{t}\right)$. Let $M_{t}=$ $\frac{1}{1-\Phi(x)} M_{t}^{\varphi}$. Then $Q_{x}^{\varphi}\left(M_{0}=1\right)=1$ and $M_{\infty}=\inf _{u \geq 0} M_{u}=\frac{1-\Phi\left(S_{\infty}\right)}{1-\Phi(x)}$.

From Proposition 3.1, decomposition (4.9) holds with $m_{t}=\frac{-\varphi\left(S_{t}\right)}{1-\Phi(x)}$. Then assumption (3.3) implies that $P\left(M_{\infty}=0\right)=1$.

Consequently 1) and 2) c) are direct consequences of Theorem 1.2 .

b) For any $u \geq g$, we have : $m_{u}=-\frac{\varphi\left(S_{\infty}\right)}{1-\Phi(x)}, M_{u}=\frac{1}{1-\Phi(x)}\left[\left(S_{\infty}-X_{u}\right) \varphi\left(S_{\infty}\right)+1-\Phi\left(S_{\infty}\right)\right]$, $\underline{M}_{u}=\underline{M}_{\infty}=\frac{1-\Phi\left(S_{\infty}\right)}{1-\Phi(x)}$ and $X_{g}=S_{\infty}$. Setting $R_{u}=X_{g}-X_{g+u}$, then the identity (4.10) implies that :

$$
R_{t}=\widetilde{\beta}_{g}-\widetilde{\beta}_{t+g}+\int_{0}^{t} \frac{d u}{R_{u}} ; t \geq 0 .
$$


Recall that $g$ is a $\left(\mathcal{G}_{t}\right)$ stopping time; hence, $\left(\widetilde{\beta}_{g}-\widetilde{\beta}_{t+g} ; t \geq 0\right)$ is a Brownian motion, independent from $\mathcal{G}_{g}$. Points a) and b) are due to the fact that (4.11) has a unique strong solution whose distribution is the law of a three dimensional Bessel process started at 0 .

We now describe the law of $\left(X_{t}\right)$ under $Q_{0}^{\lambda, \varphi}$ (we take the starting point to be 0 , for simplicity).

Theorem 4.7 Let $\varphi, \Phi$ be the functions defined by (3.11), resp. (3.10), and parameterized by the function $\psi$ satisfying (3.9).

1. Under $Q_{0}^{\lambda, \varphi}$, the r.v. $S_{\infty}$ is finite with density function $e^{-\lambda x}\left(\varphi(x)+\lambda(1-\Phi(x))=e^{-\lambda x} \psi(x)\right.$.

2. Let $g=\sup \left\{t \geq 0, X_{t}=S_{\infty}\right\}$. Then $Q_{0}^{\lambda, \varphi}(0<g<\infty)=1$ and under $Q_{0}^{\lambda, \varphi}$ :

(a) $\left(S_{\infty}-X_{t+g} ; t \geq 0\right)$ is independent of $\left(X_{t} ; 0 \leq t \leq g\right)$ and is distributed as $\left(Z_{t}^{(\lambda)} ; t \geq 0\right)$, under $P_{0}$, where

$$
Z_{t}^{(\lambda)}=X_{t}+\lambda \int_{0}^{t} \operatorname{coth}\left(\lambda Z_{u}^{(\lambda)}\right) d u
$$

(b) Conditionally on $S_{\infty}=x,\left(X_{t} ; t \leq g\right)$ is distributed as a Brownian motion with drift $\lambda$ started at 0 , and stopped when it reaches $x$.

One proof of Theorem 4.7 may be based on the theory of enlargements of filtration. This proof is similar to the proof of Theorem 4.2. Therefore we do not give it. However we will prove Theorem 4.7, using a direct approach; see Proposition 5.2 in Section 5 .

We are now able to deal with $Q_{0}^{h^{+}, h^{-}}$, where $Q_{0}^{h^{+}, h^{-}}\left(\Gamma_{t}\right)=E_{0}\left[1_{\Gamma_{t}} M_{t}^{h^{+}, h^{-}}\right] ; \Gamma_{t} \in \mathcal{F}_{t}$ and $M_{t}^{h^{+}, h^{-}}=$ $X_{t}^{+} h^{+}\left(L_{t}^{0}\right)+X_{t} h^{-}\left(L_{t}^{0}\right)+1-H\left(L_{t}^{0}\right) ; t \geq 0$. We suppose that $h^{+}, h^{-}$satisfy the conditions given in Proposition 3.11, and (3.39). Recall that the function $H$ is given by (3.36).

Theorem 4.8 1. Under $Q_{0}^{h^{+}, h^{-}}, L_{\infty}^{0}$ is a positive, finite r.v. with density function $: \frac{1}{2}\left(h^{+}+h^{-}\right)$

2. Let $g=\sup \left\{u \geq 0 ; X_{u}=0\right\}$. Then $Q_{0}^{h^{+}, h^{-}}(0<g<\infty)=1$ and under $Q_{0}^{h^{+}, h^{-}}$:

(a) the processes $\left(X_{u} ; u \leq g\right)$ and $\left(X_{u+g} ; u \geq 0\right)$ are independent,

(b) with probability $\frac{1}{2} \int_{0}^{\infty} h^{+}(u) d u$ (resp. $\frac{1}{2} \int_{0}^{\infty} h^{-}(u) d u$ ), the process $\left(X_{u+g} ; u \geq 0\right)$ (resp. $\left.\left(-X_{u+g} ; u \geq 0\right)\right)$ is distributed as a three dimensional Bessel process, started at 0.

(c) conditionally on $L_{\infty}^{0}=l,\left(X_{u} ; u \leq g\right)$ is distributed as a Brownian motion started at 0 and stopped when its local time at 0 equals $l$.

Proof. The proofs of 1), 2) a) and c) are similar to the proof of Theorem 1.6, being based on the technique of enlargements of filtrations. They are left to the reader.

However one new point has to be checked : $Q_{0}^{h^{+}, h^{-}}\left(\Gamma_{+}\right)=\frac{1}{2} \int_{0}^{\infty} h^{+}(u) d u$, where $\Gamma_{+}$is the set : $\Gamma_{+}=\left\{X_{u}>0 ; \forall u>g\right\}$.

Since either $X_{u}>0, \forall u>g$ or $X_{u}<0, \forall u>g$, then $Q_{0}^{h^{+}, h^{-}}\left(\Gamma_{+}\right)=\lim _{t \rightarrow \infty} Q_{0}^{h^{+}, h^{-}}\left(X_{t}>0\right)$.

It is easy to compute $Q_{0}^{h^{+}, h^{-}}\left(X_{t}>0\right)$ :

$$
\begin{aligned}
Q_{0}^{h^{+}, h^{-}}\left(X_{t}>0\right) & =E_{0}\left[1_{\left\{X_{t}>0\right\}} M_{t}^{h^{+}, h^{-}}\right] \\
& =E_{0}\left[X_{t}^{+} h^{+}\left(L_{t}^{0}\right)\right]+E_{0}\left[1_{\left\{X_{t}>0\right\}}\left(1-H\left(L_{t}^{0}\right)\right)\right] .
\end{aligned}
$$


The dominated convergence theorem implies that $\lim _{t \rightarrow \infty} E_{0}\left[1_{\left\{X_{t}>0\right\}}\left(1-H\left(L_{t}^{0}\right)\right)\right]=0$.

Applying Proposition 3.11 with $h^{-}=0$, we get :

$$
E_{0}\left[X_{t}^{+} h^{+}\left(L_{t}^{0}\right)\right]=\frac{1}{2} E_{0}\left[\int_{0}^{L_{t}^{0}} h^{+}(u) d u\right] .
$$

Therefore : $Q_{0}^{h^{+}, h^{-}}\left(\Gamma_{+}\right)=\frac{1}{2} \int_{0}^{\infty} h^{+}(u) d u$.

\subsection{The $Q$-process associated with the bilateral supremum and local time}

In this sub-section we study the law of $\left(X_{t}\right)$ under $Q_{0}^{\nu_{*}}$, where $\nu_{*}$ is a p.m. on $[\alpha, \infty[$, for some $\alpha>0$. Recall that $Q_{0}^{\nu_{*}}$ is the p.m. on $\left(\Omega, \mathcal{F}_{\infty}\right): Q_{0}^{\nu_{*}}\left(\Gamma_{t}\right)=E_{0}\left[1_{\Gamma_{t}} M_{t}^{\nu_{*}}\right], \Gamma_{t} \in \mathcal{F}_{t}, t \geq 0$, and $\left(M_{t}^{\nu_{*}}\right)$ is the $P_{0}$-martingale defined by (3.59).

Theorem 4.9 1. Under $Q_{0}^{\nu_{*}}, L_{\infty}^{0}$ is infinite, $X_{\infty}^{*}$ is a finite r.v., and the distribution of $X_{\infty}^{*}$ is $\nu_{*}$.

2. For any $t>0$, under $Q_{0}^{\nu_{*}},\left|X_{t}\right|<X_{\infty}^{*}$.

3. Let $\left(\mathcal{G}_{t}\right)$ be the smallest filtration satisfying the usual conditions such that for any $t \geq 0$, $\mathcal{G}_{t}$ contains $\mathcal{F}_{t} \vee \sigma\left(X_{\infty}^{*}\right)$. Then there exists $\left(\widetilde{B}_{t}\right)_{t \geq 0}$ a $\left(Q_{0}^{\nu_{*}},\left(\mathcal{G}_{t}\right)\right)$-Brownian motion started at 0 , such that:

$$
X_{t}=\widetilde{B}_{t}-\int_{0}^{t} \frac{\operatorname{sgn}\left(X_{s}\right)}{X_{\infty}^{*}-\left|X_{s}\right|} d s .
$$

Moreover :

$$
X_{\infty}^{*}-\left|X_{t}\right|=X_{\infty}^{*}+\widehat{B}_{t}+\int_{0}^{t} \frac{d s}{X_{\infty}^{*}-\left|X_{s}\right|}-L_{t}^{0}
$$

where $\left(\widehat{B}_{t}\right)_{t \geq 0}$ is the $\left(Q_{0}^{\nu_{*}},\left(\mathcal{G}_{t}\right)\right)$-Brownian motion : $\widehat{B}_{t}=-\int_{0}^{t} \operatorname{sgn}\left(X_{s}\right) d \widetilde{B}_{s}$.

Theorem 5.3 in section 5 will generalize Theorem 4.9 replacing $\left(X_{\infty}^{*}, L_{\infty}^{0}\right)$ by $\left(S_{\infty}, I_{\infty}, L_{\infty}^{0}\right)$.

The proof of Theorem 4.9 is divided in two parts, which are separated by Lemma 4.10 .

\section{First part of the proof of Theorem 4.9}

For simplicity $M$ stands for $M^{\nu_{*}}$, and $Q_{0}$ for $Q_{0}^{\nu_{*}}$. Recall :

$$
M_{t}=\int_{X_{t}^{*}}^{\infty}\left(1-\frac{\left|X_{t}\right|}{a}\right) e^{L_{t}^{0} / a} \nu_{*}(d a)=\int_{0}^{\infty}\left(1-\frac{\left|X_{t \wedge T_{a}^{*}}\right|}{a}\right) e^{L_{t \wedge T_{a}^{*}}^{0} / a} \nu_{*}(d a), \quad t \geq 0 .
$$

Let us start with the proofs of 1) and 2).

a) Let $t$ and $c$ be two positive real numbers. We have :

$$
Q_{0}\left(X_{t}^{*}>c\right)=Q_{0}\left(T_{c}^{*}<t\right)=E_{0}\left[1_{\left\{T_{c}^{*}<t\right\}} M_{t}\right]=E_{0}\left[1_{\left\{T_{c}^{*}<t\right\}} M_{T_{c}^{*}}\right] .
$$

By (4.15) we have :

$$
M_{T_{c}^{*}}=\int_{c}^{\infty}\left(1-\frac{c}{a}\right) e^{L_{T_{c}^{*}}^{0} / a} \nu_{*}(d a) .
$$

Then,

$$
Q_{0}\left(X_{\infty}^{*}>c\right)=\int_{c}^{\infty}\left(1-\frac{c}{a}\right) E_{0}\left[e^{L_{T_{c}^{*}}^{0} / a}\right] \nu_{*}(d a) .
$$


The r.v. $L_{T_{c}^{*}}^{0}$ is exponentially distributed :

$$
P_{0}\left(L_{T_{c}^{*}}^{0}>\lambda\right)=e^{-\lambda / c}
$$

Consequently if $a>c$, then $E_{0}\left[e^{L_{T_{c}^{*}}^{0} / a}\right]=\frac{a}{a-c}$ and $Q_{0}\left(X_{\infty}^{*}>c\right)=\nu_{*}(] c,+\infty[)$. This proves that the law of $X_{\infty}^{*}$ under $Q_{0}$ is $\nu_{*}$.

b) By a similar method we get :

$$
Q_{0}\left(L_{\infty}^{0}>l\right)=E_{0}\left[M_{\tau_{l}}\right]=\int_{0}^{\infty} e^{l / a} P_{0}\left(X_{\tau_{l}}^{*}<a\right) \nu_{*}(d a)
$$

Since $P_{0}\left(X_{\tau_{l}}^{*}<a\right)=P_{0}\left(T_{a}^{*}>\tau_{l}\right)=P_{0}\left(L_{T_{a}^{*}}^{0}>l\right)=e^{-l / a}$, then $Q_{0}\left(L_{\infty}^{0}>l\right)=\int_{0}^{\infty} \nu_{*}(d a)=1$, for any $l \geq 0$. This implies that $Q_{0}\left(L_{\infty}^{0}=\infty\right)=1$.

c) Let $g=\sup \left\{t \geq 0,\left|X_{t}\right|=X_{\infty}^{*}\right\}$. Let $t>0$ be fixed and $\sigma$ be the stopping time : $\sigma=\inf \{s>$ $\left.t ;\left|X_{s}\right|>X_{t}^{*}\right\}$. We claim that $Q_{0}(\sigma<\infty)=1$, this will imply that $g=\infty, Q_{0}$ a.s.

Let $n \geq 1$. Then :

$$
Q_{0}(\sigma \leq t+n)=E_{0}\left[1_{\{\sigma \leq t+n\}} M_{t+n}\right]=E_{0}\left[1_{\{\sigma \leq t+n\}} M_{\sigma}\right] .
$$

Observing that $X_{\sigma}^{*}=\left|X_{\sigma}\right|=X_{t}^{*}$ and $P_{0}(\sigma<+\infty)=1$ and taking $n \rightarrow \infty$, in (4.18), we obtain :

$$
Q_{0}(\sigma<\infty)=E_{0}\left[M_{\sigma}\right]=\int_{0}^{\infty} E_{0}\left[1_{\left\{a>X_{t}^{*}\right\}}\left(1-\frac{X_{t}^{*}}{a}\right) e^{L_{\sigma}^{0} / a}\right] \nu_{*}(d a) .
$$

On $\left\{\sigma<T_{0} \circ \theta_{t}\right\}, L_{\sigma}^{0}=L_{t}^{0}$, hence :

$$
E_{0}\left[e^{L_{\sigma}^{0} / a} 1_{\left\{\sigma<T_{0} \circ \theta_{t}\right\}} \mid \mathcal{F}_{t}\right]=e^{L_{t}^{0} / a} \frac{\left|X_{t}\right|}{X_{t}^{*}} .
$$

On $\left\{\sigma>T_{0} \circ \theta_{t}\right\}$, we have $L_{\sigma}^{0}=L_{t}^{0}+L_{T_{z}^{*}}^{0} \circ \theta_{T_{0} \circ \theta_{t}}$ with $z=X_{t}^{*}$, consequently :

$$
\begin{aligned}
E_{0}\left[e^{L_{\sigma}^{0} / a} 1_{\left\{\sigma>T_{0} \circ \theta_{t}\right\}} \mid \mathcal{F}_{t}\right] & =\left(1-\frac{\left|X_{t}\right|}{X_{t}^{*}}\right) e^{L_{t}^{0} / a} \int_{0}^{\infty} e^{l / a} e^{-l / z} \frac{d l}{z} \\
& =\frac{X_{t}^{*}-\left|X_{t}\right|}{X_{t}^{*}\left(1-\frac{X_{t}^{*}}{a}\right)} e^{L_{t}^{0} / a} .
\end{aligned}
$$

Finally :

$$
\begin{aligned}
Q_{0}(\sigma<\infty) & =E_{0}\left[\int_{X_{t}^{*}}^{\infty}\left(1-\frac{\left|X_{t}\right|}{a}\right) e^{L_{t}^{0} / a} \nu_{*}(d a)\right] \\
& =E_{0}\left[M_{t}\right]^{=}=1 .
\end{aligned}
$$

Point 3) of Theorem 4.9 will be proved via the initial enlargement of the original filtration with the r.v. $X_{\infty}^{*}$. To apply Theorem 1 of $\left[18\right.$ we need to compute $Q_{0}\left(X_{\infty}^{*}>c \mid \mathcal{F}_{t}\right), c>0, t>0$.

Lemma 4.10 Let $c>0$, and $t \geq 0$, then:

$$
\begin{aligned}
Q_{0}\left(X_{\infty}^{*} \geq c \mid \mathcal{F}_{t}\right) & =\int_{c}^{\infty} e^{L_{t \wedge T_{c}^{*}}^{0} / a}\left(1-\frac{\left|X_{t \wedge T_{c}^{*}}\right|}{a}\right) \frac{1}{M_{t \wedge T_{c}^{*}}} \nu_{*}(d a) \\
& =\nu_{*}\left(\left[c,+\infty[)+\int_{0}^{t} \widetilde{\lambda}_{s} d B_{s}\right.\right.
\end{aligned}
$$


with

$$
\begin{aligned}
& \widetilde{\lambda}_{s}=\frac{-\operatorname{sgn}\left(X_{s}\right)}{M_{s}^{2}} 1_{\left\{X_{s}^{*}<c\right\}} \int_{c}^{\infty} e^{L_{s}^{0} / a}\left\{\frac{1}{a} \int_{X_{s}^{*}}^{\infty} e^{L_{s}^{0} / b} \nu_{*}(d b)-\int_{X_{s}^{*}}^{\infty} \frac{e^{L_{s}^{0} / b}}{b} \nu_{*}(d b)\right\} \nu_{*}(d a), \\
& B_{t}=X_{t}-\int_{0}^{t} J_{s}^{\nu_{*}} d s
\end{aligned}
$$

$\left(J_{s}^{\nu_{*}}\right)$ being defined by (3.62).

Proof of Lemma 4.10 1) Let $\Gamma_{t} \in \mathcal{F}_{t}$ and $u>t$. We decompose $Q_{0}\left(\Gamma_{t} \cap\left\{X_{u}^{*} \geq c\right\}\right)$ as follows :

$$
Q_{0}\left(\Gamma_{t} \cap\left\{X_{u}^{*} \geq c\right\}\right)=Q_{0}\left(\Gamma_{t} \cap\left\{T_{c}^{*} \leq t\right\}\right)+Q_{0}\left(\Gamma_{t} \cap\left\{T_{c}^{*}>t, X_{u}^{*} \geq c\right\}\right) .
$$

Obviously : $\Gamma_{t} \cap\left\{T_{c}^{*}>t, X_{u}^{*} \geq c\right\}=\Gamma_{t}^{\prime} \cap\left\{T_{c}^{*} \leq u\right\}$ where $\Gamma_{t}^{\prime}=\Gamma_{t} \cap\left\{T_{c}^{*}>t\right\} \in \mathcal{F}_{T_{c}^{*} \wedge t}$. Consequently :

$$
Q_{0}\left(\Gamma_{t}^{\prime} \cap\left\{T_{c}^{*} \leq u\right\}\right)=E_{0}\left[1_{\Gamma_{t}^{\prime} \cap\left\{T_{c}^{*} \leq u\right\}} M_{u}\right]=E_{0}\left[1_{\Gamma_{t}^{\prime} \cap\left\{T_{c}^{*} \leq u\right\}} M_{T_{c}^{*}}\right]
$$

Using (4.15) to determine $M_{T_{c}^{*}}$ and taking $u \rightarrow \infty$, we get :

$$
Q_{0}\left(\Gamma_{t} \cap\left\{X_{\infty}^{*} \geq c\right\}\right)=Q_{0}\left(\Gamma_{t} \cap\left\{T_{c}^{*} \leq t\right\}\right)+\int_{c}^{\infty}\left(1-\frac{c}{a}\right) E_{0}\left[1_{\Gamma_{t}^{\prime}} e^{L_{T_{c}^{*}}^{0} / a}\right] \nu_{*}(d a) .
$$

To compute $E_{0}\left[1_{\Gamma_{t}^{\prime}} e^{L_{T_{c}^{*}}^{0} / a}\right]$ we proceed as in part c) of the proof of the first part of Theorem 4.9. On $\left\{T_{0} \circ \theta_{t}>T_{c}^{*} \circ \theta_{t}\right\} \cap \Gamma_{t}^{\prime}$, we have $L_{T_{c}^{*}}^{0}=L_{t}^{0}$ and :

$$
E_{0}\left[e^{L_{T_{c}^{*}}^{0} / a} \mid \mathcal{F}_{t}\right]=e^{L_{t}^{0} / a} \frac{\left|X_{t}\right|}{c}
$$

On $\left\{T_{0} \circ \theta_{t}<T_{c}^{*} \circ \theta_{t}\right\} \cap \Gamma_{t}^{\prime}$, we have $L_{T_{c}^{*}}^{0}=L_{t}^{0}+L_{T_{c}^{*}}^{0} \circ \theta_{T_{0} \circ \theta_{t}}$.

Using moreover (4.17) we deduce that on $\Gamma_{t}^{\prime}$,

$$
E_{0}\left[e^{L_{T_{c}^{*}}^{0} / a} 1_{\left\{T_{0} \circ \theta_{t}<T_{c}^{*} \circ \theta_{t}\right\}} \mid \mathcal{F}_{t}\right]=e^{L_{t}^{0} / a}\left(\frac{c-\left|X_{t}\right|}{c}\right) \frac{1}{1-c / a} .
$$

Then :

$$
\begin{aligned}
E_{0}\left[1_{\Gamma_{t}^{\prime}} e^{L_{T_{c}^{*}}^{0} / a}\right]=\frac{1}{1-c / a} E_{0}\left[1_{\Gamma_{t}^{\prime}} e^{L_{t}^{0} / a}\left(1-\frac{\left|X_{t}\right|}{a}\right)\right] \\
Q_{0}\left(\Gamma_{t} \cap\left\{X_{\infty}^{*} \geq c\right\}\right)=Q_{0}\left(\Gamma_{t} \cap\left\{T_{c}^{*} \leq t\right\}\right)+\int_{c}^{\infty} E_{0}\left[1_{\Gamma_{t}^{\prime}} e^{L_{t}^{0} / a}\left(1-\frac{\left|X_{t}\right|}{a}\right)\right] \nu_{*}(d a) \\
=Q_{0}\left(\Gamma_{t} \cap\left\{T_{c}^{*} \leq t\right\}\right)+E_{Q_{0}}\left[\int_{c}^{\infty} 1_{\Gamma_{t} \cap\left\{T_{c}^{*}>t\right\}} e^{L_{t}^{0} / a}\left(1-\frac{\left|X_{t}\right|}{a}\right) \frac{1}{M_{t}} \nu_{*}(d a)\right]
\end{aligned}
$$

The previous expression may be simplified, using (4.16) :

$$
Q_{0}\left(\Gamma_{t} \cap\left\{X_{\infty}^{*} \geq c\right\}\right)=E_{Q_{0}}\left[\int_{c}^{\infty} 1_{\Gamma_{t}} e^{L_{t \wedge T_{c}^{*}}^{0} / a}\left(1-\frac{\left|X_{t \wedge T_{c}^{*}}\right|}{a}\right) \frac{1}{M_{t \wedge T_{c}^{*}}} \nu_{*}(d a)\right] .
$$

This leads to (4.19).

2) Let $Z^{1}$ and $Z^{2}$ be two $\left(Q_{0},\left(\mathcal{F}_{t}\right)\right)$ continuous semimartingales. We write :

$$
Z^{1} \stackrel{f v}{\equiv} Z^{2}
$$

if $Z^{1}-Z^{2}$ is a continuous process with finite variation and $Z_{0}^{1}=Z_{0}^{2}$. 
It will be convenient to use this notion of congruence, which we shall apply as follows : if $\left(M_{t}\right)$ is a continuous local martingale, with $M_{0}=0$, and $M \stackrel{f v}{=} 0$, then $\left(M_{t}\right)$ is identically 0 .

Due to Theorem 3.18, the process $\left(B_{t}\right)$ defined by $(4.22)$ is a $\left(Q_{0},\left(\mathcal{F}_{t}\right)\right)$ - Brownian motion started at 0 . In particular :

$$
X_{t} \stackrel{f v}{=} B_{t}
$$

We have successively :

$$
\begin{aligned}
& \left(1-\frac{\left|X_{t}\right|}{a}\right) e^{L_{t}^{0} / a} \stackrel{f v}{=} 1-\frac{1}{a} \int_{0}^{t} \operatorname{sgn}\left(X_{s}\right) e^{L_{s}^{0} / a} d B_{s}, \\
& M_{t} \stackrel{f v}{=} \int_{0}^{\infty}\left\{1-\frac{1}{a} \int_{0}^{t} 1_{\left\{X_{s}^{*}<a\right\}} \operatorname{sgn}\left(X_{s}\right) e^{L_{s}^{0} / a} d B_{s}\right\} \nu_{*}(d a) \\
& \stackrel{f v}{=} 1-\int_{0}^{t} \operatorname{sgn}\left(X_{s}\right)\left(\int_{X_{s}^{*}}^{\infty} \frac{e^{L_{s}^{0} / a}}{a} \nu_{*}(d a)\right) d B_{s} .
\end{aligned}
$$

Let $Z^{1}$ and $Z^{2}$ be two $\left(Q_{0},\left(\mathcal{F}_{t}\right)\right)$ semimartingales, the classical rule of stochastic calculus gives :

$$
d \frac{Z_{t}^{1}}{Z_{t}^{2}} \stackrel{f v}{=} \frac{1}{Z_{t}^{2}} d Z_{t}^{1}-\frac{Z_{t}^{1}}{\left(Z_{t}^{2}\right)^{2}} d Z_{t}^{2}
$$

Choosing $Z_{t}^{1}=\left(1-\frac{\left|X_{t}\right|}{a}\right) e^{L_{t}^{0} / a}$ and $Z_{t}^{2}=M_{t}$, we obtain :

$$
\left(1-\frac{\left|X_{t}\right|}{a}\right) \frac{e^{L_{t}^{0} / a}}{M_{t}} \stackrel{f v}{=} 1-\int_{0}^{t} \operatorname{sgn}\left(X_{s}\right) e^{L_{s}^{0} / a}\left\{\frac{1}{a M_{s}}-\left(1-\frac{\left|X_{s}\right|}{a}\right) \frac{1}{M_{s}^{2}} \int_{X_{s}^{*}}^{\infty} \frac{e^{L_{s}^{0} / b}}{b} \nu_{*}(d b)\right\} d B_{s}
$$

Since $\left(Q_{0}\left(X_{\infty}^{*} \geq c \mid \mathcal{F}_{t}\right)\right)_{t \geq 0}$ is a $\left(Q_{0},\left(\mathcal{F}_{t}\right)\right)$-martingale, the identity 4.19) implies :

$$
\begin{gathered}
Q_{0}\left(X_{\infty}^{*} \geq c \mid \mathcal{F}_{t}\right)=\int_{c}^{\infty}\left[1-\int_{0}^{t} \operatorname{sgn}\left(X_{s}\right) e^{L_{s}^{0} / a} 1_{\left\{X_{s}^{*}<c\right\}}\right. \\
\left.\times\left\{\frac{1}{a M_{s}}-\left(1-\frac{\left|X_{s}\right|}{a}\right) \frac{1}{M_{s}^{2}} \int_{X_{s}^{*}}^{\infty} \frac{e^{L_{s}^{0} / b}}{b} \nu_{*}(d b)\right\} d B_{s}\right] \nu_{*}(d a) .
\end{gathered}
$$

As a result $Q_{0}\left(X_{\infty}^{*} \geq c \mid \mathcal{F}_{t}\right)=\nu_{*}\left(\left[c,+\infty[)+\int_{0}^{t} \widetilde{\lambda}_{s} d B_{s}\right.\right.$, where :

$$
\tilde{\lambda}_{s}=\frac{-\operatorname{sgn}\left(X_{s}\right)}{M_{s}^{2}} 1_{\left\{X_{s}^{*}<c\right\}} \int_{c}^{\infty} e^{L_{s}^{0} / a}\left\{\frac{M_{s}}{a}-\left(1-\frac{\left|X_{s}\right|}{a}\right) \int_{X_{s}^{*}}^{\infty} \frac{e^{L_{s}^{0} / b}}{b} \nu_{*}(d b)\right\} \nu_{*}(d a) .
$$

Formula 4.20 is a direct consequence of 4.15$)$.

\section{Second part of the proof of Theorem 4.9 (i.e. point 3)}

Obviously Lemma 4.10 may be written as follows :

$$
Q_{0}\left(X_{\infty}^{*} \geq c \mid \mathcal{F}_{t}\right)=\lambda_{t}\left(1_{[c, \infty[}\right)
$$

with the kernel $\lambda_{t}$ satisfying :

$$
\lambda_{t}\left(\left[c, \infty[)=\nu_{*}\left(\left[c, \infty[)+\int_{0}^{t} \lambda_{s}^{0}\left(\left[c, \infty[) d B_{s},\right.\right.\right.\right.\right.\right.
$$


where :

$$
\lambda_{s}^{0}(d a)=-\frac{\operatorname{sgn}\left(X_{s}\right)}{M_{s}^{2}} 1_{\left\{X_{s}^{*}<a\right\}} e^{L_{s}^{0} / a}\left[\frac{1}{a} \int_{X_{s}^{*}}^{\infty} e^{L_{s}^{0} / b}\left(1-\frac{a}{b}\right) \nu_{*}(d b)\right] \nu_{*}(d a) .
$$

The relation (4.19) directly implies that:

$$
\lambda_{t}(d a)=\frac{1}{M_{t}} 1_{\left\{X_{t}^{*}<a\right\}} e^{L_{t}^{0} / a}\left[1-\frac{\left|X_{t}\right|}{a}\right] \nu_{*}(d a) .
$$

Consequently :

$$
\lambda_{t}^{0}(d a)=\Theta(a, t) \lambda_{t}(d a)
$$

with :

$$
\Theta(a, t)=-\frac{\operatorname{sgn}\left(X_{t}\right)}{M_{t}\left(a-\left|X_{t}\right|\right)} \int_{X_{t}^{*}}^{\infty} e^{L_{t}^{0} / b}\left(1-\frac{a}{b}\right) \nu_{*}(d b) .
$$

The relations (4.28)- (4.30) allow to apply Theorem 1 of [18] (see also [5]) : there exists $\left(\widetilde{B}_{t}\right)_{t \geq 0}$ a $\left(Q_{0},\left(\mathcal{G}_{t}\right)\right)$-Brownian motion started at 0 such that :

$$
B_{t}=\widetilde{B}_{t}+\int_{0}^{t} \Theta\left(X_{\infty}^{*}, s\right) d s
$$

where $\left(\mathcal{G}_{t}\right)$ denotes the smallest filtration satisfying the usual conditions such that for any $t \geq 0$, the $\sigma$-field $\mathcal{F}_{t} \vee \sigma\left(X_{\infty}^{*}\right)$ is included in $\mathcal{G}_{t}$.

Using moreover (4.22), $\left(X_{t}\right)$ is seen to admit the decomposition : $X_{t}=\widetilde{B}_{t}+\int_{0}^{t} \xi_{s} d s$, with :

$$
\xi_{s}=\Theta\left(X_{\infty}^{*}, s\right)+J_{s}^{\nu_{*}} .
$$

Using successively (4.30), 3.62) and (4.15), we get :

$$
\begin{aligned}
\xi_{s} & =\frac{-\operatorname{sgn}\left(X_{s}\right)}{M_{s}\left(X_{\infty}^{*}-\left|X_{s}\right|\right)}\left[\int_{X_{s}^{*}}^{\infty} e^{L_{s}^{0} / b}\left(1-\frac{X_{\infty}^{*}}{b}\right) \nu_{*}(d b)+\left(X_{\infty}^{*}-\left|X_{s}\right|\right) \int_{X_{s}^{*}}^{\infty} e^{L_{s}^{0} / b} \frac{1}{b} \nu_{*}(d b)\right] \\
& =\frac{-\operatorname{sgn}\left(X_{s}\right)}{M_{s}\left(X_{\infty}^{*}-\left|X_{s}\right|\right)} \int_{X_{s}^{*}}^{\infty^{*}}\left(1-\frac{\left|X_{s}\right|}{b}\right) e^{L_{s}^{0} / b} \nu_{*}(d b)=\frac{-\operatorname{sgn}\left(X_{s}\right)}{X_{\infty}^{*}-\left|X_{s}\right|} .
\end{aligned}
$$

This proves (4.13).

Applying the Tanaka formula : $\left|X_{t}\right|=\int_{0}^{t} \operatorname{sgn}\left(X_{s}\right) d X_{s}+L_{t}^{0}$ and the relation (4.13) lead to (4.14).

\subsection{The $Q$-process associated with the down-crossings}

In this last section we are interested in the law of $\left(X_{t}\right)$ under $Q_{0}^{\downarrow, G}$. We have already introduced some notation concerning down-crossings in section 3.4. Let us briefly recall the main objects involved in this study. $D_{t}$ is the number of down-crossings from $b$ to $a(b>a)$, up to time $t$, and $(G(n))_{n>0}$ is a decreasing sequence of positive real numbers, satisfying $G(0)=1$ and $\lim _{n \rightarrow \infty} G(n)=0$. Let $\left(M_{t}^{\downarrow, G}\right)_{t \geq 0}$ be the positive $P_{0}$-martingale associated with $(G(n))_{n \geq 0}$ as defined in Proposition 3.20. $Q_{0}^{\downarrow, G}$ will denote the p.m. on the canonical space $\left(\Omega, \mathcal{F}_{\infty}\right): Q_{0}^{\downarrow, G}\left(\Gamma_{t}\right)=E_{0}\left[1_{\Gamma_{t}} M_{t}^{\downarrow, G}\right], \Gamma_{t} \in \mathcal{F}_{t}$.

Theorem 4.11 1. Under $Q_{0}^{\downarrow, G}$, the r.v. $D_{\infty}$ is finite and

$$
Q_{0}^{\downarrow, G}\left(D_{\infty}=n\right)=G(n)-G(n+1) ; n \geq 0 .
$$


2. Let $g$ and $\bar{g}$ be the two random times :

$$
g=\inf \left\{t \geq 0 ; D_{t}=D_{\infty}\right\} ; \bar{g}=\inf \left\{t>g ; X_{t}=b\right\}
$$

Then : $Q_{0}^{\downarrow, G}(0<g<\infty)=1$ and $Q_{0}^{\downarrow, G}(\bar{g}<\infty)=1 / 2$.

3. Under $Q_{0}^{\downarrow, G}$ and conditionally on $\{\bar{g}<\infty\}$ :

(a) $\left(X_{u} ; 0 \leq u \leq g\right),\left(X_{g+u} ; 0 \leq u \leq \bar{g}-g\right)$ and $\left(X_{\bar{g}+u} ; u \geq 0\right)$ are independent,

(b) $\left(2 b-a-X_{g+u} ; 0 \leq u \leq \bar{g}-g\right)$ is distributed as a three dimensional Bessel process, started at $2(b-a)$ and stopped at its first hitting time of level $b-a$,

(c) $\left(X_{u+\bar{g}}-a ; u \geq 0\right)$ is distributed as a three dimensional Bessel process started at $b-a$.

4. Under $Q_{0}^{\downarrow, G}$ and conditionally on $\{\bar{g}=\infty\}$ :

(a) $\left(X_{u} ; 0 \leq u \leq g\right)$, and $\left(X_{g+u} ; u \geq 0\right)$ are independent,

(b) $\left(2 b-a-X_{g+u} ; u \geq 0\right)$ is distributed as a three dimensional Bessel process, started at $2(b-a)$ and conditioned to be greater than $b-a$.

5. Under $Q_{0}^{\downarrow, G}$ and conditionally to $\left\{D_{\infty}=n\right\},\left(X_{u} ; 0 \leq u \leq g\right)$ is distributed as a Brownian motion started at 0 , and stopped at the first time $\left(\sigma_{2 n}\right)$ when the number of down-crossings equals $n$.

Proof. a) Recall that $\left(\sigma_{n}\right)_{n \geq 0}$ is the sequence of stopping times defined by (3.68)-(3.71). Using the definition of $Q_{0}^{\downarrow, G}$ and the optional stopping theorem, we obtain :

$$
\begin{aligned}
Q_{0}^{\downarrow, G}\left(D_{t} \geq n\right) & =Q_{0}^{\downarrow, G}\left(\sigma_{2 n} \leq t\right)=E_{0}\left[1_{\left\{\sigma_{2 n} \leq t\right\}} M_{t}^{\downarrow, G}\right] \\
& =E_{0}\left[1_{\left\{\sigma_{2 n} \leq t\right\}} M_{\sigma_{2 n}}^{\downarrow, D}\right] .
\end{aligned}
$$

But relation (3.75) implies that $M_{\sigma_{2 n}}^{\downarrow, G}=G(n)$. Consequently, taking $t \rightarrow \infty$, in the previous identity leads to : $Q_{0}^{\downarrow}, G\left(D_{\infty} \geq n\right)=G(n)$. Hence $Q_{0}^{\downarrow, G}\left(D_{\infty}=n\right)=G(n)-G(n+1)$. Since $\lim _{n \rightarrow \infty} G(n)=0$, then $Q_{0}^{\downarrow, G}\left(D_{\infty}<\infty\right)=1$.

b) The proof of 2-5 of Theorem 4.11 makes use of the progressive enlargement of filtrations technique. Since we have already developed this approach in the setting of Theorems 4.2 and 4.8, we limit ourselves to state the main steps without giving details.

We have :

$$
Z_{t}:=Q_{0}^{\downarrow, G}\left(g>t \mid \mathcal{F}_{t}\right)=\frac{G\left(1+D_{t}\right)}{M_{t}^{\downarrow}, G} ; \forall t \geq 0 .
$$

Theorem 3.21 says that the process $\left(B_{t}\right)$ defined by relation $(3.84)$ is a $Q_{0}^{\downarrow, G}$ - Brownian motion, started at 0 .

It is convenient for the sequel to introduce :

$$
m_{s}=\frac{G\left(1+D_{s}\right)-G\left(D_{s}\right)}{2(b-a)}\left(1_{\left\{\sigma_{2 D_{s}}+T_{b} \circ \theta_{\sigma_{2 D s}}>s\right\}}-1_{\left\{\sigma_{2 D_{s}}+T_{b} \circ \theta_{\sigma_{2 D s}}<s\right\}}\right) .
$$

Consequently :

$$
\begin{gathered}
X_{t}=B_{t}+\int_{0}^{t} \frac{m_{s}}{M_{s}^{\downarrow, G}} d s, \\
M_{t}^{\downarrow, G}=M_{0}^{\downarrow, G}+\int_{0}^{t} m_{s} d X_{s} .
\end{gathered}
$$


Applying Itô's formula, we obtain :

$$
\begin{aligned}
Z_{t} & =1-\int_{0}^{t} \frac{G\left(1+D_{s}\right)}{\left(M_{s}^{\downarrow, G}\right)^{2}} m_{s} d X_{s}+\int_{[0, t]} \frac{d_{s} G\left(1+D_{s}\right)}{M_{s}^{\downarrow, G}} \\
& =1-\int_{0}^{t} \frac{G\left(1+D_{s}\right)}{\left(M_{s}^{\downarrow, G}\right)^{2}} m_{s} d B_{s}-\int_{0}^{t} \frac{G\left(1+D_{s}\right)}{\left(M_{s}^{\downarrow, G}\right)^{3}}\left(m_{s}\right)^{2} d B_{s}+\int_{[0, t]} \frac{d_{s} G\left(1+D_{s}\right)}{M_{s}^{\downarrow, G}} .
\end{aligned}
$$

Let $\left(\mathcal{G}_{t}\right)_{t>0}$ be the smallest filtration, containing $\left(\mathcal{F}_{t}\right)_{t>0}$, satisfying the usual conditions and such that $g$ is a $\left(\mathcal{G}_{t}\right)_{t \geq 0}$-stopping time. Then there exists a $\left(Q_{0}^{\downarrow, G},\left(\mathcal{G}_{t}\right)_{t \geq 0}\right)$-Brownian motion $\left(\widehat{B}_{t}\right)$ started at 0 , such that :

$$
B_{t}=\widehat{B}_{t}+\int_{0}^{t \wedge g} \frac{d<Z, X>_{s}}{Z_{s}}-\int_{t \wedge g}^{t} \frac{d<Z, X>_{s}}{1-Z_{s}} .
$$

Since :

$$
\left.d<Z, X>_{s}=-\frac{G\left(1+D_{s}\right)}{\left(M_{s}^{\downarrow}, G\right.}\right)^{2} m_{s} d s
$$

we have :

$$
X_{t}=\widehat{B}_{t}+\int_{t \wedge g}^{t} \frac{m_{s}}{M_{s}^{\downarrow, G}-G\left(1+D_{s}\right)} d s .
$$

It is easy to compute the drift term, via (3.74) :

$$
\frac{m_{s}}{M_{s}^{\downarrow, G}-G\left(1+D_{s}\right)}=\left\{\begin{array}{l}
\frac{1}{X_{s}+a-2 b} \text { on }\left\{\sigma_{2 D_{s}}+T_{b} \circ \theta_{\sigma_{2 D_{s}}}>s\right\}, \\
\frac{1}{X_{s}-a} \text { otherwise. }
\end{array}\right.
$$

In particular :

$$
X_{t}=\widehat{B}_{t}+\int_{t \wedge g}^{t \wedge \bar{g}} \frac{d s}{X_{s}+a-2 b}+\int_{t \wedge \bar{g}}^{t} \frac{d s}{X_{s}-a} ; t \geq 0 .
$$

Having obtained these results, points 2-5 of Theorem 4.11 can now be proved. The details are left to the reader.

\section{$5 \quad$ A direct approach to study the canonical process under $Q$}

To explain the goal of this section let us start with Case 1. For simplicity we restrict ourselves to $x=0$. Theorem 4.6 leads us to consider $Q_{0}^{(y)}$, the law of $\left(X_{t}\right)_{t \geq 0}$ conditionally on $S_{\infty}=y$. Recall that under $Q_{0}^{(y)}$,

- $\left(X_{t} ; t \leq T_{y}\right)$ is a Brownian motion started at 0 , and considered up to its first hitting time of $y$,

- $\left(y-X_{t+T_{y}} ; t \geq 0\right)$ is a three dimensional Bessel process started at 0 .

Therefore, Theorem 4.6 may be summarized as follows :

$$
Q_{0}^{\varphi}(\cdot)=\int_{0}^{\infty} Q_{0}^{(y)}(\cdot) \varphi(y) d y .
$$

This motivated us to prove (5.1) directly without any enlargement of filtration.

Proposition 5.1 Let $\varphi:\left[0,+\infty\left[\longrightarrow \mathbb{R}_{+}\right.\right.$as in Proposition 3.1, satisfying (3.3). Then (5.1) holds. 
Proof. 1) Let $0 \leq a<b$ and $\varphi(x)=\frac{1}{b-a} 1_{[a, b]}(x)$. Then :

$$
\Phi(x)=\int_{0}^{x} \varphi(t) d t=\frac{x \wedge b-a}{b-a} 1_{\{x \geq a\}} .
$$

Consequently :

$$
M_{t}^{\varphi}=1_{\left\{S_{t}<a\right\}}+\frac{b-X_{t}}{b-a} 1_{\left\{a \leq S_{t} \leq b\right\}} ; t \geq 0 .
$$

Then using Girsanov's theorem and Proposition 3.1, it is easy to check that $\left(X_{t}\right)_{t \geq 0}$ solves :

$$
X_{t}=\beta_{t}-\int_{0}^{t} \frac{1}{b-X_{u}} 1_{\left\{a \leq S_{u} \leq b\right\}} d u
$$

where $\left(\beta_{t} ; t \geq 0\right)$ is a $Q_{0}^{\varphi}$-Brownian motion started at 0 .

Consequently, under $Q_{0}^{\varphi}$ :

- $\left(X_{t} ; 0 \leq t \leq T_{a}\right)$ is distributed as a Brownian motion started at 0 , and stopped at its first hitting of $a$,

- $\left(b-X_{t+T_{a}} ; t \geq 0\right)$ is distributed as a three dimensional Bessel process started at $b-a$.

2) To prove (5.1), it is convenient to give an adequate description of the p.m. $\int_{0}^{\infty} Q_{0}^{(y)}(\cdot) \varphi(y) d y$. Let $\xi$ be a r.v. independent of $\left(X_{t}\right)_{t \geq 0}$ and uniformly distributed on $[a, b]$. Then under $\int_{0}^{\infty} Q_{0}^{(y)}(\cdot) \varphi(y) d y$ :

- $\left(X_{t} ; 0 \leq t \leq T_{\xi}\right)$ is distributed as $\left(X_{t} ; 0 \leq t \leq T_{\xi}\right)$ under $P_{0}$,

- $\left(\xi-X_{t+T_{\xi}} ; t \geq 0\right)$ is distributed as a three dimensional Bessel process started at 0 .

In particular $\left(X_{t} ; t \leq T_{a}\right)$ has the same distribution under either $Q_{0}^{\varphi}$ or $\int_{0}^{\infty} Q_{0}^{(y)}(\cdot) \varphi(y) d y$.

3) Let $R_{b-a}$ be a three dimensional Bessel process started at $b-a$, and $g$ be the unique time $t$ such that $R_{b-a}(t)=\inf _{u \geq 0} R_{b-a}(u)$. Then :

- $\left(R_{b-a}(t+g)-\inf _{u \geq 0} R_{b-a}(u) ; t \geq 0\right)$ is independent of $\left(R_{b-a}(t) ; 0 \leq t \leq g\right)$ and is distributed as a three dimensional Bessel process started at 0 ,

- $\inf _{u \geq 0} R_{b-a}(u)$ is uniformly distributed on $[0, b-a]$, and conditionally on $\inf _{u \geq 0} R_{b-a}(u)=x,\left(R_{b-a}(t) ; 0 \leq\right.$ $t \leq g)$ is distributed as a Brownian motion started at $b-a$, and stopped when it reaches $x$.

This implies that the law of $\left(X_{t+T_{a}} ; t \geq 0\right)$ is the same under either $Q_{0}^{\varphi}$ or $\int_{0}^{\infty} Q_{0}^{(y)}(\cdot) \varphi(y) d y$.

4) Let $\varphi: \mathbb{R}_{+} \mapsto \mathbb{R}_{+}$be a Borel function such that $\int_{0}^{\infty} \varphi(y) d y=1$. It is clear that $Q_{0}^{\varphi}$ is equal to $\widetilde{Q}_{0}^{\varphi}$, with :

$$
\widetilde{Q}_{0}^{\varphi}\left(\Gamma_{t}\right):=E_{0}\left[1_{\Gamma_{t}}\left(\left(S_{t}-X_{t}\right) \varphi\left(S_{t}\right)+\int_{S_{t}}^{\infty} \varphi(y) d y\right)\right], t \geq 0, \Gamma_{t} \in \mathcal{F}_{t}
$$

But we have proved that the two p.m. $\widetilde{Q}_{0}^{\varphi}$ and $\int_{0}^{\infty} Q_{0}^{(y)}(\cdot) \varphi(y) d y$ coincide when $\varphi(x)=\frac{1}{b-a} 1_{[a, b]}(x)$. Since $\psi \longrightarrow \widetilde{Q}_{0}^{\psi}$ and $\psi \longrightarrow \int_{0}^{\infty} Q_{0}^{(y)}(\cdot) \psi(y) d y$ are linear (with respect to convex combinations), then

$$
\widetilde{Q}_{0}^{\varphi}(\cdot)=\int_{0}^{\infty} Q_{0}^{(y)}(\cdot) \varphi(y) d y
$$


holds for any elementary function $\varphi$ of the type : $\varphi=\sum_{i} c_{i} 1_{\left[a_{i}, b_{i}\right]}$. Approximating a continuous function with compact support by a sequence of stepwise constant functions of the previous type, permits to prove (5.1), $\varphi$ being a positive continuous function with compact support in $[0,+\infty[$. The Riesz representation theorem allows to extend (5.2) and (5.1) to any Borel and positive $\varphi$, the details are left to the reader.

We have already observed that if we take $\lambda=0$, then the Kennedy martingale $M^{0, \varphi}$ coincides with $M^{\varphi}$. This leads us to generalize Proposition 5.1. We give (see Proposition 5.2 below) a direct proof of Theorem 4.7 via a disintegration of the p.m. $Q_{0}^{\lambda, \varphi}$. Formally taking $\lambda \rightarrow 0$ in Proposition 5.2 permits to recover Proposition 5.1. However the proofs of Propositions 5.2 and 5.1 are different.

Let $\lambda>0, x>0$ and $Q_{0}^{\lambda,(x)}$ be the unique p.m. on the canonical space such that:

- $\left(X_{t} ; 0 \leq t \leq T_{x}\right)$ is distributed as a Brownian motion with drift $\lambda$, started at 0 , and stopped at its first hitting time of $x$,

- $\left(x-X_{t+T_{x}} ; t \geq 0\right)$ is distributed as $\left(Z_{t}^{(\lambda)} ; t \geq 0\right)$ under $P_{0}$, where :

$$
Z_{t}^{(\lambda)}=X_{t}+\lambda \int_{0}^{t} \operatorname{coth}\left(\lambda Z_{u}^{(\lambda)}\right) d u
$$

We observe that :

$$
Q_{0}^{(x)}=\lim _{\lambda \rightarrow 0} Q_{0}^{\lambda,(x)} .
$$

Proposition 5.2 Let $\varphi, \Phi$ be the functions defined by (3.11), resp. (3.10), and parametrized by the function $\psi$ satisfying $\int_{0}^{\infty} \psi(z) e^{-\lambda z} d z=1$. Then :

$$
Q_{0}^{\lambda, \varphi}(\cdot)=\int_{0}^{\infty} Q_{0}^{\lambda,(y)}(\cdot) e^{-\lambda y} \psi(y) d y
$$

Proof. Let $\Gamma \in \mathcal{F}_{\infty}$. Using the definition of $Q_{0}^{\lambda, \varphi}$ and (3.20), we have :

$$
Q_{0}^{\lambda, \varphi}(\Gamma)=E_{P_{x}^{(-\lambda)}}\left[\frac{\psi\left(S_{\infty}\right)}{2 \lambda} e^{\lambda S_{\infty}} 1_{\Gamma}\right] .
$$

Conditioning on $S_{\infty}$ and using (3.19) leads to :

$$
Q_{0}^{\lambda, \varphi}(\Gamma)=\int_{0}^{\infty} e^{-\lambda y} \psi(y) P_{x}^{(-\lambda)}\left(\Gamma \mid S_{\infty}=y\right) d y .
$$

The equality : $Q_{0}^{\lambda,(y)}(\Gamma)=P_{x}^{(-\lambda)}\left(\Gamma \mid S_{\infty}=y\right)$ follows directly from the well-known theorem of Williams [17.

We now investigate the law of $\left(X_{t}\right)$ under $Q_{0}^{\nu}$, where $\nu$ is a p.m. on $[\alpha, \infty[\times[\alpha, \infty[$, for some $\alpha>0$. The p.m. $Q_{0}^{\nu}$ has been already introduced in Theorem 3.18 and $\left(M_{t}^{\nu}\right)$ is the $P_{0}$-martingale defined in Proposition 3.16. Note that the penalization result has been proved (see Theorem 3.18) for the triplet $\left(S_{t}, I_{t}, L_{t}^{0}\right)$ but we have only described the law of $\left(X_{t}\right)$ under $Q_{0}^{\nu_{*}}$; this p.m. being associated with the two dimensional process $\left(X_{t}^{*}=S_{t} \vee I_{t}, L_{t}^{0}\right)$. Hence Theorem 5.3 below, generalizes Theorem 4.9 . Moreover, its proof hinges on a disintegration of $Q_{0}^{\nu}$ and does not use enlargement of filtration . 
Let $Q_{0}^{s, i}$ be the p.m. on $\left(\Omega, \mathcal{F}_{\infty}\right)$ defined as the law of $\left(Y_{t}^{s, i}\right)$ under $P_{0}$, where $s, i \geq 0$ and $\left(Y_{t}^{s, i}\right)$ is the solution of the following SDE :

$$
Y_{t}=X_{t}-\int_{0}^{t} \frac{1}{s-Y_{u}} 1_{\left\{Y_{u}>0\right\}} d u+\int_{0}^{t} \frac{1}{i+Y_{u}} 1_{\left\{Y_{u}<0\right\}} d u
$$

It will cause no confusion to keep the same letter $Q$ to designate the p.m used in both Proposition 5.2 above and Theorem 5.3 below, since the first p.m.'s is always indexed by $(\lambda,(y))$ and the second p.m. by $(s, i)$.

Coming back to (5.7), F. Knight (可) already considered the process $\left(Y_{t}^{s, s}\right)$ and called it the "Brownian taboo process". Intuitively it is a Brownian motion conditioned on never reaching $\pm s$, the taboo levels. Likewise, from (5.7), it can be proved that :

- the process $\left(Y_{t}^{s, i}\right)$ takes its values in $]-i, s[$,

- $\sup _{t \geq 0} Y_{t}^{s, i}=s$ and $\inf _{t \geq 0} Y_{t}^{s, i}=-i$.

These properties may be also proved via the classification of boundary points $s,-i$ of the diffusion process $\left(Y_{t}^{s, i}\right)$ (see for instance $([11]$, section V 50-51).

Theorem 5.3 1. Under $Q_{0}^{\nu}, L_{\infty}^{0}$ is infinite, $S_{\infty}, I_{\infty}$ are finite r.v., and the distribution of $\left(S_{\infty}, I_{\infty}\right)$ is $\nu$.

2. We have:

$$
Q_{0}^{\nu}(\cdot)=\int_{\left(\left[0,+\infty[)^{2}\right.\right.} Q_{0}^{s, i}(\cdot) \nu(d s, d i)
$$

Remark 5.4 The probabilistic interpretation of the disintegration property (5.8) is the following : conditionally on $S_{\infty}=s, I_{\infty}=i$, the law of $\left(X_{t}\right)$ under $Q_{0}^{\nu}$ coincides with the law of $\left(Y_{t}^{s, i}\right)$ under $P_{0}$.

Proof of Theorem 5.3 1) To determine the law of $\left(S_{\infty}, I_{\infty}\right)$ and prove that $L_{\infty}^{0} \equiv \infty$ a.s., we can proceed as in the proof of Theorem 4.9. The details are left to the reader.

2) Let us prove (5.8). Let $s, i \geq 0$ fixed, and $b$ be the function :

$$
b(x)=-\frac{1}{s-x} 1_{\{0<x<s\}}+\frac{1}{i+x} 1_{\{-i<x<0\}} .
$$

The Girsanov theorem implies that :

$$
Q_{0}^{s, i}{ }_{\mid \mathcal{F}_{t}}=\Theta_{t} 1_{\left\{t<T_{s} \wedge T_{-i}\right\}} P_{0} \mid \mathcal{F}_{t},
$$

where

$$
\Theta_{t}=\exp \left\{\int_{0}^{t} b\left(X_{u}\right) d X_{u}-\frac{1}{2} \int_{0}^{t} b\left(X_{u}\right)^{2} d u\right\} .
$$

Applying the Itô-Tanaka formula we obtain :

$$
\begin{aligned}
\ln \left(s-X_{t}^{+}\right) & =\ln (s)-\int_{0}^{t} \frac{1}{s-X_{u}} 1_{\left\{X_{u}>0\right\}} d X_{u}-\frac{1}{2} \int_{0}^{t} \frac{1}{\left(s-X_{u}\right)^{2}} 1_{\left\{X_{u}>0\right\}} d u-\frac{1}{2 s} L_{t}^{0}, \\
\ln \left(i-X_{t}^{-}\right) & =\ln (i)+\int_{0}^{t} \frac{1}{i+X_{u}} 1_{\left\{X_{u}<0\right\}} d X_{u}-\frac{1}{2} \int_{0}^{t} \frac{1}{\left(i+X_{u}\right)^{2}} 1_{\left\{X_{u}<0\right\}} d u-\frac{1}{2 i} L_{t}^{0},
\end{aligned}
$$

where $t<T_{s} \wedge T_{-i}$.

It follows that :

$$
\Theta_{t}=\left(1-\frac{X_{t}^{+}}{s}\right)\left(1-\frac{X_{t}^{-}}{i}\right) \exp \left\{\frac{1}{2}\left(\frac{1}{s}+\frac{1}{i}\right) L_{t}^{0}\right\}
$$


Summarizing previous calculations, we get :

$$
Q_{0}^{s, i} \mid \mathcal{F}_{t}=\left(1-\frac{X_{t}^{+}}{s}\right)\left(1-\frac{X_{t}^{-}}{i}\right) \exp \left\{\frac{1}{2}\left(\frac{1}{s}+\frac{1}{i}\right) L_{t}^{0}\right\} 1_{\left\{t<T_{s} \wedge T_{-i}\right\}} P_{0 \mid \mathcal{F}_{t}}
$$

Integrating with respect to $\nu(d s, d i)$ implies directly $(5.8)$, since the martingale $\left(M_{t}^{\nu}\right)$ is defined by (3.51).

Our Theorem 5.3 provides a short proof of Lemma 3.19. It is actually possible to demonstrate directly Lemma 3.19, however tedious calculations are necessary.

Let $a, b>0, \nu(d s, d i):=\delta_{a}(d s) \otimes \delta_{b}(d i)$ and $M_{t}=M_{t}^{\nu}$.

Applying the definition (3.51) of $\left(M_{t}\right)$, we have :

$$
M_{t}=\frac{1}{a b}\left(a-X_{t}^{+}\right)\left(b-X_{t}^{-}\right) e^{c L_{t}^{0}} 1_{\left\{t \leq T_{a} \wedge T_{-b}\right\}} ; t \geq 0,
$$

with $c=\frac{1}{2}\left(\frac{1}{a}+\frac{1}{b}\right)$.

Due to the definition of $Q_{0}^{a, b}$, we get :

$$
\begin{aligned}
E_{Q_{0}^{a, b}}\left[\frac{1}{\left(a-X_{t}^{+}\right)\left(b-X_{t}^{-}\right)}\right] & =E_{0}\left[\frac{1}{\left(a-X_{t}^{+}\right)\left(b-X_{t}^{-}\right)} M_{t}\right] \\
& =\frac{1}{a b} E_{0}\left[e^{c L_{t}^{0}} 1_{\left\{t \leq T_{a} \wedge T_{-b}\right\}}\right] .
\end{aligned}
$$

Consequently :

$$
\lim _{t \rightarrow \infty} E_{0}\left[e^{c L_{t}^{0}} 1_{\left\{t \leq T_{a} \wedge T_{-b}\right\}}\right]=a b \lim _{t \rightarrow \infty} E_{Q_{0}^{\nu}}\left[\frac{1}{\left(a-X_{t}^{+}\right)\left(b-X_{t}^{-}\right)}\right] .
$$

But under $Q_{0}^{\nu},\left(X_{t}\right)$ is a recurrent diffusion. It is easy to compute its invariant density function $g$ since this function solves :

$$
\begin{cases}\frac{1}{2} g "(x)+\frac{g^{\prime}(x)}{a-x}+\frac{g(x)}{(a-x)^{2}}=0 & \text { if } x>0, \\ \frac{1}{2} g "(x)-\frac{g^{\prime}(x)}{b+x}+\frac{g(x)}{(b+x)^{2}}=0 & \text { if } x<0\end{cases}
$$

Finally :

$$
g(x)=\frac{3}{(a+b) a^{2} b^{2}}\left[b^{2}(a-x)^{2} 1_{\{0<x<a\}}+a^{2}(b+x)^{2} 1_{\{-b<x<0\}}\right] .
$$

We observe that :

$$
\int_{\mathbb{R}} \frac{1}{\left(a-x^{+}\right)\left(b-x^{-}\right)} g(x) d x=\frac{3}{2 a b} .
$$

In particular the integral above is finite and

$$
\lim _{t \rightarrow \infty} E_{Q_{0}^{\nu}}\left[\frac{1}{\left(a-X_{t}^{+}\right)\left(b-X_{t}^{-}\right)}\right]=\frac{3}{2} .
$$

This finishes the proof of Lemma 3.19, since :

$$
\lim _{t \rightarrow \infty} E_{0}\left[e^{c L_{t}^{0}} 1_{\left\{t \leq T_{a} \wedge T_{-b}\right\}}\right]=\frac{3}{2} .
$$

Remark 5.5 1. It is easy to deduce from the previous analysis that

$$
\lim _{t \rightarrow \infty} E_{0}\left[e^{\alpha L_{t}^{0}} 1_{\left\{t \leq T_{a} \wedge T_{-b}\right\}}\right]=\left\{\begin{array}{cl}
\infty & \text { if } \alpha>c, \\
0 & \text { if } \alpha<c .
\end{array}\right.
$$


2. Lemma 3.19 may be generalized as follows. Let $\left(Y_{t}\right)$ be Walsh's Brownian motion with parameters $\left(p_{i}\right)_{1 \leq i \leq n}$, where $0<p_{i}<1$ and $\sum_{i=1}^{n} p_{i}=1$. We recall (see Walsh's original paper [10], and also [4] and [3] for detailed constructions), that this process takes its values in a union of half-lines $I_{1}, \cdots, I_{n}$ such that $\cap_{i=1}^{n} I_{i}=\{0\}$. Heuristically, this process :

- moves as a one-dimensional Brownian motion inside each $I_{i}$,

- when it reaches 0 , it chooses at random, with probability $p_{i}$, to evolve in $I_{i}$.

Now, the statement of Lemma 3.19 may be extended in the following manner :

$$
\lim _{t \rightarrow \infty} E\left[e^{\alpha L_{t}^{0}} \prod_{i=1}^{n} 1_{\left\{S_{t}^{i} \leq a_{i}\right\}}\right]=\left\{\begin{array}{cl}
0 & \text { if } \alpha<c \\
3 / 2 & \text { if } \alpha=c \\
\infty & \text { if } \alpha>c
\end{array}\right.
$$

$$
\text { with } c:=\sum_{i=1}^{n} \frac{p_{i}}{a_{i}}
$$

\section{$6 \quad$ Further developments}

In this section, we sketch a number of results which shall appear in [15].

For simplicity, we shall only discuss here Case 1.

1. To prove our main limit result, rather than considering $E\left[\varphi\left(S_{t}\right) \mid \mathcal{F}_{s}\right]$, as $t \rightarrow \infty$, we study $P\left(\Gamma_{s} \mid S_{t}=y\right]$, which is shown to converge as $t \rightarrow \infty$ towards $Q_{0}^{(y)}\left(\Gamma_{s}\right)$.

2. Also, we study the speed of convergence of $Q_{0, t}^{\varphi}\left(\Gamma_{s}\right)$, where $Q_{0, t}^{\varphi}$ denotes the p.m. $Q_{0, t}^{F}$ defined by (1.6) with $F_{t}=\varphi\left(S_{t}\right)$. More precisely, suppose that $\varphi$ satisfies moreover $\int_{0}^{\infty} \varphi(t) t^{2} d t<\infty$, we then prove that there exists a $P_{0}$-martingale $\left(N_{t}^{\varphi}\right)$ such that :

$$
\frac{E\left[1_{\Gamma_{s}} \varphi\left(S_{t}\right)\right]}{E\left[\varphi\left(S_{t}\right)\right]}-E\left[1_{\Gamma_{s}} M_{s}^{\varphi}\right]_{t \rightarrow \infty} \frac{E\left[1_{\Gamma_{s}} N_{s}^{\varphi}\right]}{t}, \quad \forall \Gamma_{s} \in \mathcal{F}_{s}
$$

We obtain more generally an expansion of the left side of (6.1) in powers of $(1 / t)$, to any order.

3. Finally we consider ( [15], [14]) the same problems with $F_{t}=f\left(X_{t}, S_{t}\right)$ where $f: \mathbb{R} \times \mathbb{R}_{+} \mapsto \mathbb{R}_{+}$. We suppose :

$$
\bar{f}:=\int_{\mathbb{R}} d x \int_{x_{+}}^{\infty}(2 y-x) f(x, y) d y<\infty .
$$

Denote $\widehat{f}=1 / \bar{f}$.

Then we establish that $Q_{0, t}\left(\Gamma_{s}\right)$ converges to $\widehat{f} E_{0}\left[1_{\Gamma_{s}} M_{s}^{\varphi}\right], t \rightarrow \infty$, for any $\Gamma_{s} \in \mathcal{F}_{s}$ where $\varphi$ is given by :

$$
\varphi(y)=\widehat{f} \int_{\mathbb{R}} d x \int_{y \vee x_{+}}^{\infty} f(x, z) d z+\int_{-\infty}^{y} f(x, y)(y-x) d x .
$$

\section{References}

[1] J. Azéma and M. Yor. Une solution simple au problème de Skorokhod. In Séminaire de Probabilités, XIII (Univ. Strasbourg, Strasbourg, 1977/78), volume 721 of Lecture Notes in Math., pages 90-115. Springer, Berlin, 1979. 
[2] J. Azéma and M. Yor. Décomposition multiplicative de certaines sous-martingales. Unpublished notes, 1991.

[3] M. Barlow, J. Pitman, and M. Yor. On Walsh's Brownian motions. In Séminaire de Probabilités, XXIII, volume 1372 of Lecture Notes in Math., pages 275-293. Springer, Berlin, 1989.

[4] M. Barlow, J. Pitman, and M. Yor. Une extension multidimensionnelle de la loi de l'arc sinus. In Séminaire de Probabilités, XXIII, volume 1372 of Lecture Notes in Math., pages 294-314. Springer, Berlin, 1989.

[5] J. Jacod. Grossissement initial, hypothèse (H'), et théorème de Girsanov. In Grossissement de filtrations : exemples et applications (Séminaire de Calcul Stochastique, Paris 1982/83), volume 1118 of Lecture Notes in Math., pages 15-35. Springer, Berlin, 1985.

[6] T. Jeulin. Semi-martingales et grossissement d'une filtration, volume 833 of Lecture Notes in Mathematics. Springer, Berlin, 1980.

[7] T. Jeulin and M. Yor. Sur les distributions de certaines fonctionnelles du mouvement brownien. In Seminar on Probability, XV (Univ. Strasbourg, Strasbourg, 1979/1980) (French), volume 850 of Lecture Notes in Math., pages 210-226. Springer, Berlin, 1981.

[8] I. Karatzas and S. E. Shreve. Brownian motion and stochastic calculus, volume 113 of Graduate Texts in Mathematics. Springer-Verlag, New York, second edition, 1991.

[9] F. B. Knight. Brownian local times and taboo processes. Trans. Amer. Math. Soc., 143:173-185, 1969.

[10] D. Revuz and M. Yor. Continuous martingales and Brownian motion, volume 293 of Grundlehren der Mathematischen Wissenschaften [Fundamental Principles of Mathematical Sciences]. Springer-Verlag, Berlin, third edition, 1999.

[11] L. C. G. Rogers and D. Williams. Diffusions, Markov processes, and martingales. Vol. 2. Cambridge Mathematical Library. Cambridge University Press, Cambridge, 2000. Itô calculus, Reprint of the second (1994) edition.

[12] B. Roynette, P. Vallois, and M. Yor. Limiting laws associated with Brownian motion perturbated by normalized exponential weights. C. R. Acad. Sci. Paris Sér. I Math., 337:667-673, 2003.

[13] B. Roynette, P. Vallois, and M. Yor. Limiting laws associated with Brownian motion perturbed by normalized exponential weights I. To appear in Studia Sci. Math. Hungar., 2004.

[14] B. Roynette, P. Vallois, and M. Yor. Pénalisations et extensions du théorème de Pitman, relatives au mouvement brownien et à son maximum unilatère. In Seminar on Probability, XXXIX (P.A. Meyer, in memoriam), Lecture Notes in Math. Springer, Berlin, 2005.

[15] B. Roynette, P. Vallois, and M. Yor. Penalizations of a Brownian motion with drift by a function of its one-sided maximum and its position, III. To appear in Periodica Hungar., 2005.

[16] J.B. Walsh. A diffusion with a discontinuous local time. In Temps locaux, volume 52-53 of Astérisque, pages 37-45. Société Mathématique de France, 1978.

[17] D. Williams. Path decomposition and continuity of local time for one-dimensional diffusions. I. Proc. London Math. Soc. (3), 28:738-768, 1974.

[18] M. Yor. Grossissement de filtrations et absolue continuité de noyaux. In Grossissement de filtrations : exemples et applications (Séminaire de Calcul Stochastique, Paris 1982/83), volume 1118 of Lecture Notes in Math., pages 6-14. Springer, Berlin, 1985. 